\title{
Effective Approaches to Extract Features and Classify Echoes in Long Ultrasound Signals from Metal Shafts
}

by

\author{
Kyungmi Lee \\ BSc., Computer Science, Pusan National University, Korea, 1993. \\ Grad. Certificate, Computer Science, University of Newcastle, Australia, 2000.
}

A thesis submitted in fulfillment of the requirements

for the degree of Doctor of Philosophy

\begin{abstract}
LVJ Griffith
School of Information and Communication Technology

Griffith University

Brisbane QLD 4111

Australia
\end{abstract}

(c) Kyungmi Lee, March 2006 


\section{Certificate of Originality}

I hereby certify that the work embodied in this thesis is the result of original research and has not been submitted for a higher degree at any other University or Institution.

(Signed)

Kyungmi Lee 


\section{Acknowledgements}

I owe a substantial debt to many people in the production of this thesis and would like to thank and acknowledge them for the support and inspiration I have received over the period of my doctoral candidature.

I would like to sincerely thank my supervisor, Professor Vladimir Estivill-Castro, for his guidance, encouragement, patience, tireless reviewing, constructive feedback, infectious enthusiasm and inspiration throughout the researching and preparation of this thesis. His support towards my participation in international conferences, and commitment to an environment with adequate equipment and facilities is deeply appreciated. This thesis would not have been possible at all without his encouragement and support.

I would also like to thank my external co-supervisor Dr Guy Cotterill from CCI Pope Pty Ltd, the industrial partner of this project, for his advice and assistance with collecting raw ultrasonic data and identifying initial data features. I also would like to express many thanks to John Perceval and Adam Van Dyck of the Product Development team of CCI Pope for their helpful collaboration. I would like to acknowledge CCI Pope Pty Ltd for providing their funding, facilities and the equipment used for field experiments.

My special thanks go to the Australian Research Council (ARC) who provided me a Ph.D. scholarship, Australian Postgraduate Award Industry (APAI), through the ARC Linkage Project (Project ID C00107362).

I would like to thank the School of Information and Communication Technology, Griffith University for providing a highly supportive research environment. I specially thank them for providing necessary computing and communication equipment during my off-campus studying. I owe a lot of the completion of my Ph.D. study to this practical support from the school. I also acknowledge the support of Griffith University Research Computing Services for providing High Performance computing services (QPSF MetaCentre Facility).

I must thank my parents, brother and sister. They understand little of what this is about 
but are proud nonetheless. Their endless love and support has also made this thesis possible.

Finally but most importantly, I am deeply and forever grateful to my husband, Ickjai and my two children, Eojin and Haanbie. My special thanks go to Ickjai for his everlasting great support and encouragement. Other special thanks to Eojin for her tolerance and kind understanding of the busy mum. Another special thanks to Haanbie for being born healthy on mum's way to her Ph.D. completeness. I dedicate this thesis to these three people with love and gratitude. 
To my husband, Ickjai (Jai), my daughter, Eojin (Jinny), and my son Haanbie. 


\title{
Publications arising from this thesis
}

\author{
International Journals with full paper refereed
}

1. Kyungmi Lee and Vladimir Estivill-Castro. "Feature extraction and gating techniques for ultrasonic shaft signal classification", Applied Soft Computing, Volume 7, Issue 1 (January 2007), pages 156-165, Elsevier, 2007.

2. Kyungmi Lee and Vladimir Estivill-Castro. "Classification Ensemble for Shaft Test Data: Empirical Evaluation.", International Journal of Simulation Systems, Volume 6, Number 10-11, pages 38-47, United Kingdom Simulation Society, 2005. ISSN 14738031.

\section{International conferences and workshops with full paper refereed}

1. Kyungmi Lee and Vladimir Estivill-Castro. "Classification of Ultrasonic Shaft Inspection Data Using Discrete Wavelet Transform”, in M. H. Hamza, editor, Proceedings of the 3rd IASTED International Conference on Artificial Intelligence and Applications, pages 673-678, Benalmadena, Spain, 2003. ACTA Press, ISBN: 0-88986-390-3, ISSN 1482-7913.

2. Kyungmi Lee and Vladimir Estivill-Castro. "Feature Extraction Techniques for Ultrasonic Shaft Signal Classification", in A. Abraham et al., editors, Proceedings of the 3rd International Conference on Hybrid Intelligent System (HIS-2003), Frontiers in Artificial Intelligence and Applications, Vol. 104, pages 479-488, Melbourne, Australia, 2003. IOS Press, ISBN 1-58603-394-8, ISSN 0922-6389.

3. Kyungmi Lee and Vladimir Estivill-Castro. "Support Vector Machine Classification of Ultrasonic Shaft Inspection Data Using Discrete Wavelet Transform", in H. Arabna and Y. Mun, editors, Proceedings of the 2004 International Conference on Machine Learning; Models, Technologies and Applications (MLMTA'04), pages 848-854, Las Vegas, Nevada, USA, 2004. CSREA Press, ISBN: 1-932415-27-0. 
4. Kyungmi Lee and Vladimir Estivill-Castro. "Classification Ensemble for Shaft Test Data: Empirical Evaluation", in M. Ishikawa et al., editors, Proceedings of the 4th International Conference on Hybrid Intelligent System (HIS-2004), pages 304-309, Kitakyushu, Japan, 2004. IEEE Computer Society, ISBN 0-7695-2291-2.

5. Kyungmi Lee and Vladimir Estivill-Castro. "A Hybrid Classification Approach to Ultrasonic Shaft Signals", in G. I. Webb and X. Yu, editors, Proceedings of the 17th Australian Joint Conference on Artificial Intelligence (AI2004), Lecture Notes in Artificial Intelligence 3339, pages 284-295, Cairns, Australia, 2004. Springer-Verlag, ISBN 3-540-24059-4, ISSN 0302-9743. 


\section{Acronyms}

- ANN - Artificial Neural Network

- AUSC - Automatic Ultrasonic Signal Classification

- BC - Bayesian Combination

- BE - Back-wall Echo

- BR - Back-wall Reflection

- CDWT - Clustered DWT (Discrete Wavelet Transform)

- CMT - Confusion Matrix Table

- CP - Central Peak positioning

- CR - CRack

- DFT - Discrete Fourier Transform

- DGT - Discrete Gabor Transform

- DS - Distribution Summation

- DWT - Discrete Wavelet Transform

- ECM - Error Cost Matrix

- FFT - Fast Fourier Transform

- FT - Fourier Transform

- IP - Initial Pulse

- ISVM - Integrated SVM classifier

- LC - Likelihood Combination

- LVQ - Learning Vector Quantization

- MC - Mode Conversion 
- ME - Main Energy computing

- MSE - Mean Square Error

- NDE - Non-Destructive Evaluation

- NDT - Non-Destructive Testing

- PNN - Probabilistic Neural Network

- PPT - Posterior Probability Table

- QMF - Quadrature Mirror Filters

- RA - RAndom positioning

- RBF - Radial Basis Function

- SE - Secondary Echo

- SNNS - Stuttgart Neural Network Simulator

- SO - Systematic echo capturing with preservation of Original neighboring grass

- STFT - Short-Time Fourier Transform

- SVM - Support Vector Machine

- SZ - Systematic echo capturing method with Zero-padding 


\section{Contents}

List of Figures $\quad$ xii

List of Tables $\quad$ xiv

1 Introduction 1

1.1 Non-Destructive Testing and Ultrasonic Flaw Testing . . . . . . . . . . . 1

1.2 Mode-Converted Echoes in A-Scan Signals from Shafts . . . . . . . . . . . . 3

1.3 Automatic Ultrasonic Signal Classification Systems . . . . . . . . . . . . . 4

1.4 Aims and Contributions . . . . . . . . . . . . . . . . . 10

1.5 Outline of the thesis . . . . . . . . . . . . . . . 13

2 Feature Extraction Schemes for Ultrasonic Signals $\quad 15$

2.1 Preprocessing of Ultrasonic Signals . . . . . . . . . . . . . . . . . . . . . 16

2.2 Extraction of Signal Features _ . . . . . . . . . . . . . . . . . 17

2.3 Fourier Analysis and FFT . . . . . . . . . . . . . . . . . 20

2.3.1 FFT coefficients using magnitude (FFT_Mag) . . . . . . . . . . . . 21

2.3.2 FFT coefficients using magnitude and phase (FFT_Magpha) . . . . . 22

2.4 Wavelet Analysis and DWT . . . . . . . . . . . . . . . . 24

2.4 .1 DWT coefficients . . . . . . . . . . . . . . 27

2.4 .2 DWT as a feature extraction scheme . . . . . . . . . . . 29

$\begin{array}{lll}3 & \text { FFT } & \text { v } \\ \text { DWT using ANN Classifiers } & 31\end{array}$

3.1 Introduction . . . . . . . . . . . . . . . . . . . . 31

3.2 Related Work . . . . . . . . . . . . . . . . . . . . . . 33

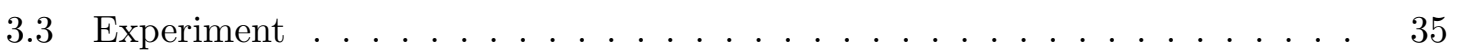


3.3.1 Overall Process . . . . . . . . . . . . . . . . . 35

3.3.2 Preprocessing and Feature Extraction . . . . . . . . . . 36

3.3 .3 ANN Classification . . . . . . . . . . . . . . . . 39

3.4 Results and Analysis . . . . . . . . . . . . . . . . . 41

3.5 Remarks . . . . . . . . . . . . . . . . . . . . 45

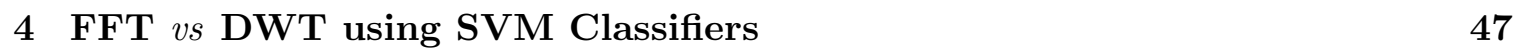

4.1 Introduction . . . . . . . . . . . . . . . . . . . . . . 47

4.2 SVM Classifier . . . . . . . . . . . . . . . . . . . . . 49

4.3 Experiment . . . . . . . . . . . . . . . . . . 51

$4.3 .1 \quad$ Overall Process . . . . . . . . . . . . . . . . . 51

4.3.2 Preprocessing and Feature Extraction . . . . . . . . . 53

4.3.3 SVM Classification . . . . . . . . . . . . . . . . . 55

4.4 Results and Analysis . . . . . . . . . . . . . . . . . . . 57

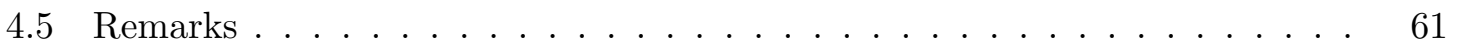

5 Echo Gating Techniques for Ultrasonic A-Scans 62

5.1 Introduction . . . . . . . . . . . . . . . . . 62

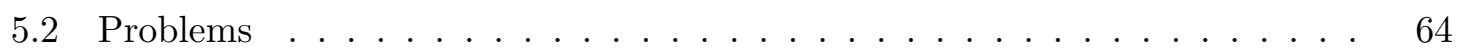

5.3 Echo Border Decision Method . . . . . . . . . . . . . 67

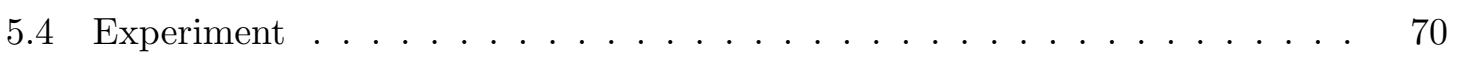

5.4 .1 Experimental setting . . . . . . . . . . . . 70

5.4 .2 Preprocessing and Feature Extraction . . . . . . . . . 71

5.4 .3 ANN Classification . . . . . . . . . . . . . . . . 73

5.4 .4 SVM classification .......................... 74

5.5 Results and Analysis . . . . . . . . . . . . . . . 75

5.6 Remarks ............................ 76

6 Ensembles for Ultrasonic Signal Classifiers $\quad 81$

6.1 Introduction . . . . . . . . . . . . . . . . . . . . 81

6.2 Combining Multi-Classifiers . . . . . . . . . . . . . . . 83

6.3 Experiment . . . . . . . . . . . . . . . . . 85

6.3 .1 Generating multiple classifiers . . . . . . . . . . . . 85

6.3 .2 Combining multiple classifiers . . . . . . . . . . . . . 89 
6.4 Results and Analysis . . . . . . . . . . . . . . . . . . 90

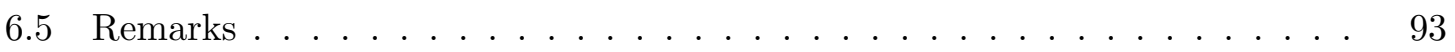

7 A Cost-Sensitive Integrated SVM Classification System for Ultrasonic Signal Tests $\quad 95$

7.1 Introduction . . . . . . . . . . . . . . . . . . 95

7.2 A Cost-Sensitive Integrated Classification System . . . . . . . . . . . . . 97

7.2.1 Posterior Probability Table and Confusion Matrix Table . . . . . . . 98

7.2.2 Cost of Misclassification Errors . . . . . . . . . . . . . . . 100

7.2 .3 Decision-Making Rule in ISVM . . . . . . . . . . . . . . . . 102

7.3 Empirical Evaluation . . . . . . . . . . . . . . . . . 104

7.4 Remarks . . . . . . . . . . . . . . . . . . 109

8 Conclusions and Future Work $\quad 111$

8.1 Summary . . . . . . . . . . . . . . . . . . . . 111

8.2 Future Work . . . . . . . . . . . . . . . . . 116

$\begin{array}{ll}\text { A ANOVA test result } & 122\end{array}$

A.1 ANOVA test for the experimental results of Chapter $3 \ldots \ldots$. . . . . 123

A.2 ANOVA test for the experimental results of Chapter $4 \ldots \ldots$. . . . . 124

A.3 ANOVA test for the experimental results of Chapter $5 \ldots \ldots \ldots$

B Grid searching results of selecting parameters for SVM models $\quad 135$

B.1 Grid searching for $(C, \gamma)$ for SVM classifiers of Chapter $4 \ldots \ldots$. . . . 135

B.2 Grid searching for $(C, \gamma)$ for SVM classifiers of Chapter $5 \ldots \ldots \ldots$

C Methods for Combining Classifiers 143

C.1 Bayesian Combination . . . . . . . . . . . . . . . . . . . . . 143

C.2 Distribution Summation . . . . . . . . . . . . . . . . 145

C.3 Likelihood Combination . . . . . . . . . . . . . . . . . . 145

$\begin{array}{ll}\text { D The result weights for three combining methods } & 147\end{array}$

$\begin{array}{lr}\text { Bibliography } & 150\end{array}$ 


\section{List of Figures}

1.1 A typical ultrasonic A-Scan system . . . . . . . . . . . . . . 2

1.2 Ultrasonic pulse generating secondary echoes and the corresponding A-Scan 4

1.3 An illustration of an automated signal classification system . . . . . . . 5

1.4 The research question and the goal of this thesis f . . . . . . . . . . 11

1.5 The categorization of our research aims corresponding to each stage of developing an AUSC system . . . . . . . . . . . . . . . . 11

2.1 Feature extraction schemes based on time domain and frequency domain . . 18

2.2 The procedure of constructing FFT_Mag and FFT_Magpha . . . . . . . . 23

2.3 Four different views of a signal depending on different domains: time-domain, frequency-domain, STFT and Wavelet analysis . . . . . . . . 26

2.4 One-dimensional DWT decomposition algorithm. . . . . . . . . . . . 28

3.1 An overall procedure diagram for the experiment for ANNs classification. . 37

3.2 The relative comparison of performance of ANNs using three different feature extraction schemes: FFT_Mag vs FFT_Magpha vs DWT. . . . . . . . . . 43

4.1 An overall procedure for the experiment for SVM classification. . . . . . . . 52

4.2 Comparison of performance of SVMs using two different feature extraction schemes: FFT vs DWT . . . . . . . . . . . . . . . . . . 59

5.1 A signal echo gated on the captured ultrasonic A-scan signal . . . . . . . 64

5.2 Two different gating methods applied to one flaw signal and their corresponding DWT coefficients . . . . . . . . . . . . . . 65

5.3 An example of a small amplitude echo and its normalized representation . . 67

5.4 The flow-chart of the procedure for deciding echo borders . . . . . . . 68

5.5 Comparison of performance of different feature extraction techniques. . . . 78 
6.1 Two methods of constructing AUSC systems: the single classifier method and the multi-classifier method . . . . . . . . . . . . . . 82

6.2 Methods of generating multi-classifiers for ensembles . . . . . . . . . . . 84

6.3 Diagram of our experiment presenting two main procedures: model-generation and model-combination . . . . . . . . . . . . 86

6.4 Overall procedure of our experiment for building an ensemble of classifiers . $\quad 87$

6.5 Bar charts for the comparison of the performance of combined models . . . 92

7.1 The overall structure of ISVM . . . . . . . . . . . . . . . . . . 98

7.2 Flow chart of the overall procedure of constructing the decision rule in ISVM. 103

7.3 The overall procedure of our experiment for the evaluation of the performance of ISVM. . . . . . . . . . . . . . . . . . . . . 105

7.4 Comparing the classification performance of FSVM, DSVM and ISVM. . . 108

8.1 A summary of the possible future extensions of this work $\ldots \ldots \ldots$ 


\section{List of Tables}

1.1 Categorization of the previous related research . . . . . . . . . . 6

3.1 The classification performance of ANNs using three different feature extraction schemes: FFT_Mag vs FFT_Magpha vs DWT. . . . . . . . . . .

3.2 Summary of ANOVA test result of Accuracy and number of required epochs for ANN models using three different feature extraction schemes: FFT_Mag vs FFT_Magpha vs DWT. . . . . . . . . . . . . . . . . . .

4.1 The classification performance of SVMs using two different feature extraction schemes: FFT vs DWT . . . . . . . . . . . . . . . .

4.2 Summary of ANOVA test result of the classification performance of SVMs using two different feature extraction schemes: FFT vs DWT. . . . . . . . .

4.3 The classification performance of ANNs and SVMs using three different feature extraction schemes with the earlier version of the test data set . . . . .

5.1 Experimental parameter setting for SVM models of DWT and clusteredDWT feature extraction schemes using five different preprocessing methods.

5.2 Classification performance of DWT and clustered-DWT feature extraction schemes using five different preprocessing methods . . . . . . . . . .

5.3 Summary of ANOVA test result of the classification performance of ANNs and SVMs using DWT-related feature extraction schemes and different preprocessing methods . . . . . . . . . . . . . . . .

6.1 Comparison of the performance of combined models $\ldots \ldots \ldots \ldots$

7.1 The classification accuracy of SVMs using different input vectors: FFT vs DWT vs FFT+DWT . . . . . . . . . . . . . . . . . . . . . . 97

7.2 The relation between CMT and PPT . . . . . . . . . . . . . 99

7.3 Error-Cost Matrix (ECM) and expected risk for each decision. . . . . . . . 101 
7.4 Expected Risk (ER) for each hypothesis on ISVM's decision and derived rules for final decision . . . . . . . . . . . . . . . . . . . 102 


\section{Abstract}

Applications of machine learning demand exploration of feature extraction methods and classifier types in order to obtain systems with reliable highest accuracy. The industrial application discussed in this thesis is the classification of ultrasonic echoes in an A-scan. The application is particularly challenging as A-scans are taken from the end of long large complex metal shafts. Although several pattern analysis and machine learning techniques have been used with success in analyzing A-scan data [43, 89], they are typically in the context of very short signals produced from machine parts like plate surfaces or pipe surfaces. Those cases are usually much simpler; in particular, the task reduces to detecting the existence of an echo (indicating a fault in the material). When signals for testing come from long shafts, however, a major problem of mode-converted reflection emerges. These reflections are echoes that do not correspond to real faults (cracks), nor to characteristics in the shaft. These mode-converted echoes may cause misjudgement of the position of cracks on shafts as some critical faint echoes from a cracked surface may lie somewhere among the multiple secondary echoes. Consequences of misclassification are catastrophic with enormous cost in downtime, consequential damage to associate equipment and potential injury to personnel [23].

The problem is then, to discriminate efficiently the different types of reflectors amongst the large volumes of digitalized ultrasonic shaft defect information. As the relationship between ultrasonic signal characteristics and flaw classes is not straightforward, we need to identify and extract informative sets of signal features from which classification might be performed more efficiently and accurately.

Among various methods for extracting signal features, the Fast Fourier Transform (FFT) is a useful scheme for extracting frequency-domain signal features [23, 62]. This seems natural when dealing with ultrasound since the traditional representation of these types of signals is by mathematical Fourier series that identify physically meaningful features, like frequency and phase. But recent studies on the ultrasonic flaw classification employ the Discrete Wavelet Transform (DWT) as part of their feature extraction scheme. DWT provides effective signal compression and time-frequency presentation [69, 86]. Many researchers have compared these two feature extraction schemes (FFT and DWT), and most 
comparisons showed a superiority of DWT to FFT in discriminating the type of flaw (or its non-existence) $[74,78,90]$. However, these previous reported studies have compared the DWT based features with the FFT with limited feature components. Typically, short signals have been reported, with little attention to phase components of FFT sequences. This thesis is the first study analyzing feature extraction in more complex ultrasonic signals from shafts. In particular, we introduce a new FFT-based feature extraction scheme FFT_Magpha which effectively represents both magnitude and phase components of FFT sequences. By employing this state-of-the-art FFT feature extraction scheme, we have more extension and reliability in the investigation about the feasibility of FFT as a better feature extraction scheme than other types of feature extraction schemes such as DWT.

On the other hand, the time-variance problem exhibited in DWT has resulted in reservations about its wide acceptance even though DWT coefficients provide effective time-frequency representation of non-stationary signals, and thus are considered useful features for input into classifiers. To solve this, we study a new preprocessing technique for time-domain A-scans, which offer consistent extraction of a segment of the signal from long signals that occur in the NDT of shafts. We compare the performance of this new echo-gating technique with other previously developed methods and investigate that we can use DWT more efficiently as a feature extraction scheme for ultrasonic signal classification by employing this new method in the preprocessing stage. In addition, our investigation in this thesis finds the potential of DWT to be a more reliable feature extraction scheme, through the more stable classification results in different runs of cross validation tests than the results produced in the tests using FFT-based feature extraction scheme. This potential is especially beneficial for the practical NDT for shafts, where we can train a classifier with arbitrary training data and then use the classifier for in-field ultrasonic shaft signal test.

We also demonstrate the superiority of using DWT as the feature extraction scheme in the ultrasonic shaft signal classification involving not only ANN but also SVM. These results dissipate any doubt that the DWT feature extraction methodology is too far suited for ANN which has been popularly employed previously in many similar experimental scenarios. Through these experimental comparisons employing various learning algorithms, we find a certain facility when specific classes of echoes are concerned with different combinations of feature extraction (FFT or DWT) and classifier (ANN or SVM), though DWT is superior to FFT and SVM is superior to ANN in terms of the overall classification accuracy. This 
finding leads into a hybrid classifier that will improve overall performance by giving more weight to the more trustworthy sub-classifier.

Based on those experimental analysis, we design an Integrated SVM classifier (ISVM) which is a combined classification system efficiently employing benefits from each of two SVM classifiers using two feature extraction schemes, FFT and DWT. The outcomes of a classifier based on FFT is not totally dismissed in this system although the DWT-based classifier has been shown to be superior. This property of ISVM enables us to combine classifiers considering the misclassification cost, to obtain a more informative classification for its application in the field. We also explore the diverse possibilities of heterogeneous and homogeneous ensembles by combining the classifiers along the dimension of feature extraction mechanism, along the dimension of combination method and along the dimension of type of classifier. The experimental result suggests guidelines for designing an integrated multi-classifier system for shaft test data by way of selectively employing the combining structure. 


\section{Chapter 1}

\section{Introduction}

This thesis studies feature extraction and the discrimination of echoes in ultrasonic flaw detection for long metal shafts. In this chapter we introduce the subject area of ultrasonic flaw testing and summarize the motivation behind the thesis. The chapter then summarizes the contributions of the thesis and outlines its contents.

\subsection{Non-Destructive Testing and Ultrasonic Flaw Testing}

Machine parts suffer stress and strain that result in failure. Predicting the time of failure has proven very challenging. Thus, it is very important to measure the operating characteristics of machine parts and detect any defects in them throughout their life. In many industrial applications, materials or machine parts are to be tested without any kind of damage or disruption, and this type of testing is called Non-Destructive Testing (NDT) or NonDestructive Evaluation (NDE). NDT techniques enable us to check the performance of machine systems in a manner which will not impair their future usefulness.

Operators of critical plants are particularly interested in early detection of conditions that lead to in-service failures of rotating shafts and fixed pins because they are susceptible to failures without apparent warning. NDT techniques allow in-situ detection of fatigue cracking which usually precedes failures in shafts and pins. 
One of the most commonly used NDT techniques is ultrasonic inspection, which employs a piezoelectric transducer that produces mechanical oscillations inside the inspected material, and acquires the reflected ultrasonic wave from discontinuities in the material $[97,86]$. One ultrasonic method, called A-scan, displays reflected sound amplitude as a function of time (depth) for a particular location in the test specimen. A waveform indicating a flaw (defect) will have additional high amplitude pulses between the front and back surfaces. The location and size of the intermediate pulses are generally assumed to be related to the size and/or the orientation of the defect [64]. A typical ultrasonic A-Scan system is represented in Figure 1.1. An ultrasonic A-Scan system for testing shafts is particularly advantageous when it is comprised of a portable computer to enable it to house the entire system in a single unit so that it can be used as an in-situ testing system in various industrial fields without disassembling shafts or disrupting their operation. Short bursts of elastic wave energy are injected into a machine part by a transducer coupled to an electronic pulserreceiver. Parameters such as the filter and gain profiles are also controlled and set up in this system. Based on the selected parameters, the signal received by the transducer is filtered and amplified by the chosen filter option and gain profile. The ultrasonic signals are then collected and digitized via a data acquisition card. The collected signals are presented in the format of an A-Scan which is a plot of signal voltage versus time for one transducer position.

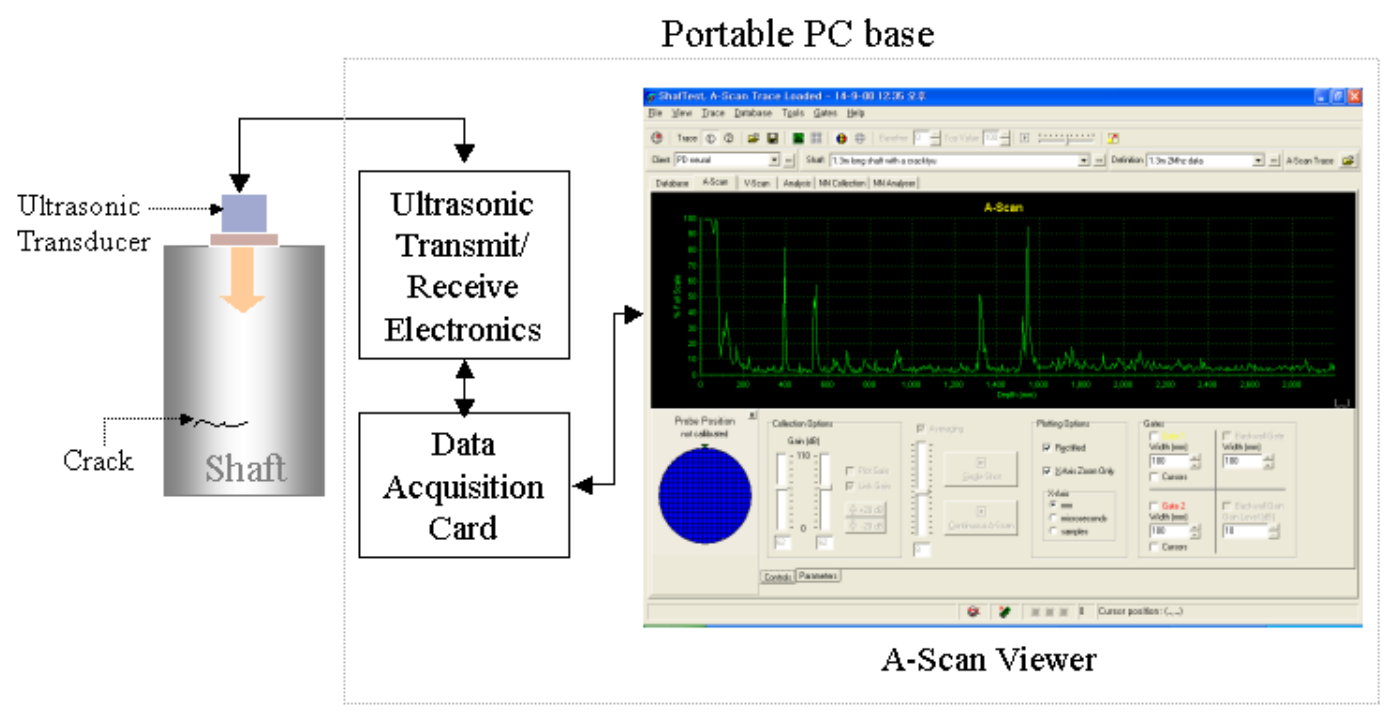

Figure 1.1: A typical ultrasonic A-Scan system. 


\subsection{Mode-Converted Echoes in A-Scan Signals from Shafts}

Though an A-Scan generally displays somewhat recognizable waveforms indicating defects, not all defect indications are easy, especially when the part geometry becomes complicated, when the defect location is close to either the front or back surfaces or when a very high level of structural noise is induced $[2,6]$. Especially, A-scan signals taken from shafts are more complicated to interpret than A-scans from other machine parts like plate surfaces or pipe surfaces which produce shorter and simpler signals [23].

Because A-scans for a shaft are taken from the end of a lengthy shaft, an ultrasonic pulse can travel many paths echoing off the machined surfaces. If the pulse hits the side of the shaft, mode conversion occurs and a shear wave propagates. This wave can then go through another mode conversion back to a compression wave, which can then be reflected back to the source and receiver. These are called secondary echoes or mode-converted echoes and they result from multiple instances of mode conversion between a shear wave and a compression wave as the ultrasonic wave travels the length of the shaft in various angles from the source depending on the diameter of the shaft. ${ }^{1}$ Figure 1.2 (a) illustrates the principle behind the generation of mode-converted echoes by showing some illustrative lines of wave propagation of ultrasonic pulse on a lengthy shaft. Figure 1.2 (b) shows a typical A-Scan of a cylindrical block such as the one shown in Figure 1.2 (a).

Moreover, most shafts are often more complex than a plain solid cylinder. Most machine shafts have multiple sections with different diameters or more complex geometry such as splines, oil ways, pin drill holes, bolt holes or key ways. Thus, the many reflections from these machined features render it difficult to correctly attribute signals to defects. When cracking due to fatigue appears in a shaft, the returned signal represented as an A-Scan reflects this defect by additional peaks and echoes. These signals can be very complex and difficult to interpret, especially when the shaft has such complex geometric features.

\footnotetext{
${ }^{1}$ When a compression wave goes through a shallow reflection angle some of the energy is converted to a shear wave. These shear waves are transmitted at approximately $33^{\circ}$ and can subsequently go through multiple reflections but must ultimately be converted back to a compression wave through one of these reflections in order to be detected, i.e. a compression wave probe is only sensitive to the reception of a compression wave vibration. As a consequence, only certain beam paths result in a mode-converted echo which depend primarily on the diameter of the shaft. The separation between multiple secondary echoes is approx. $0.76 \times$ diameter [49].
} 
(a)

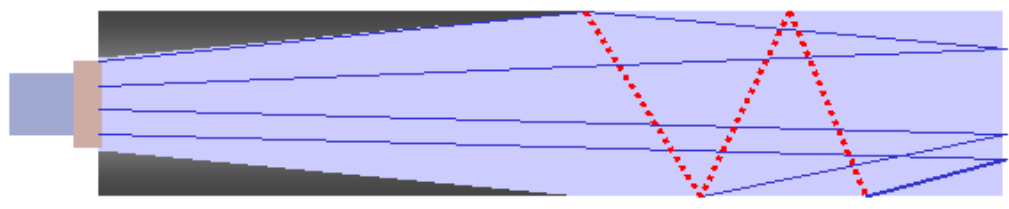

(b)

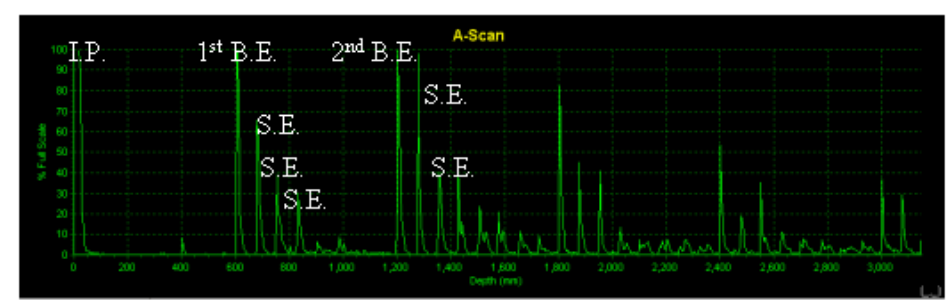

Figure 1.2: (a) Ultrasonic pulse generating mode-converted echoes in shaft testing and (b) A-Scan showing backwall echoes(also known as primary echoes) and secondary echoes(also known as mode-converted echoes). IP: initial pulse, BE: backwall echo, SE: secondary echo.

These mode-converted echoes may cause misjudgement of the position of features as some critical faint echoes from a cracked surface may lie somewhere among the multiple secondary echoes. The problem is then, to discriminate efficiently amongst the large volumes of digital information, the different types of reflectors, and classify them into a) those that correspond to design features of the metal piece (DF), b) those that correspond to flaws, cracks and other defects emerging on the shaft (CR) and c) the multiple reflections and mode-converted echoes (MC) of the two earlier cases a) and b). Among these three causes of echoes, type $\mathrm{DF}$ is considered to be more easily distinguished than other types; namely CR or MC. An issue of further concern in the field is that the signal echoes caused by CR can be confused by faked echoes caused by MC and vice versa. Consequences of both cases are catastrophic with enormous cost in downtime, consequential damage to associate equipment and potential injury to personnel [23].

\subsection{Automatic Ultrasonic Signal Classification Systems}

Conventional NDT techniques, which are based on manual, heuristic or experience-based pattern identification methods, bring about costly, lengthy and erratic analysis and thus lead to inconsistencies in results. To reduce the effect of human variability in interpretation, 
to get the consistency of response, and to analyze and interpret large volumes of signals, a variety of modern signal processing techniques and Artificial Intelligence (AI) tools have been adopted $[43,70,89]$. These approaches can be integrated as Automatic Ultrasonic Signal Classification (AUSC) systems. The overall approach used for an AUSC system consists of three major steps as shown in Figure 1.3. In the first step, we process the ultrasonic flaw signals. They are acquired in digital form, so we call them digitized data. Informative features are then extracted using various digital signal processing techniques. The set of selected features becomes the basis of decision-making for classification in the final step. We characterize the previous research on automated ultrasonic data analysis by the schemes shown in Table 1.1. This table organizes research according to its main focus and then by one of the three steps in an AUSC system.

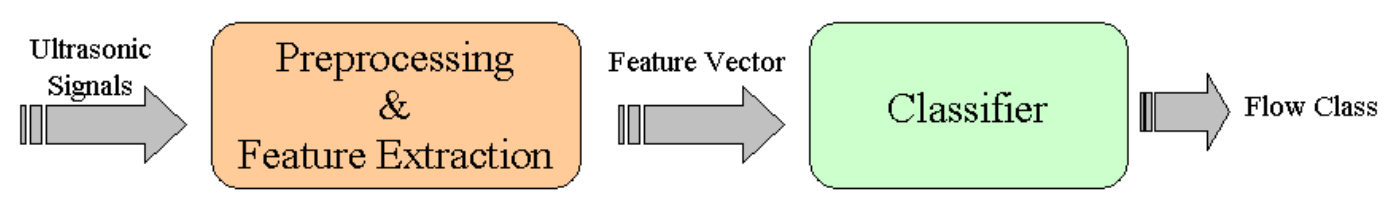

Figure 1.3: An illustration of an automated signal classification system.

In the preprocessing stage, the primary objective is to render the classification results invariant to the effects of variations in test and sample conditions including gain and frequency settings, time delays in samples signals, and other minor differences in material properties. In this specific case, two main approaches were proposed to develop a signal classification system such that the performance is reasonably insensitive to the choice of the transducer center frequency. One suggests a frequency invariance technique based on time-scaling that transforms both the center frequency and the bandwidth of the signal [74], and the other approach is based on the normalization of features by the features in a reference signal [89].

The second step involves the identification and extraction from the raw signal of those features that determine the class of the echo. The use of various digital processing techniques allows the extraction of those features. As the relationship between ultrasonic signal characteristics and flaw classes is not straightforward, the extraction of features plays a critical role in classification accuracy and this becomes the important basis of decision-making for classification [107]. A number of previous studies have proposed various sets of ultrasonic signal features chosen from the time-domain and/or the frequency-domain. The studies 
Table 1.1: Categorization of the previous related research.

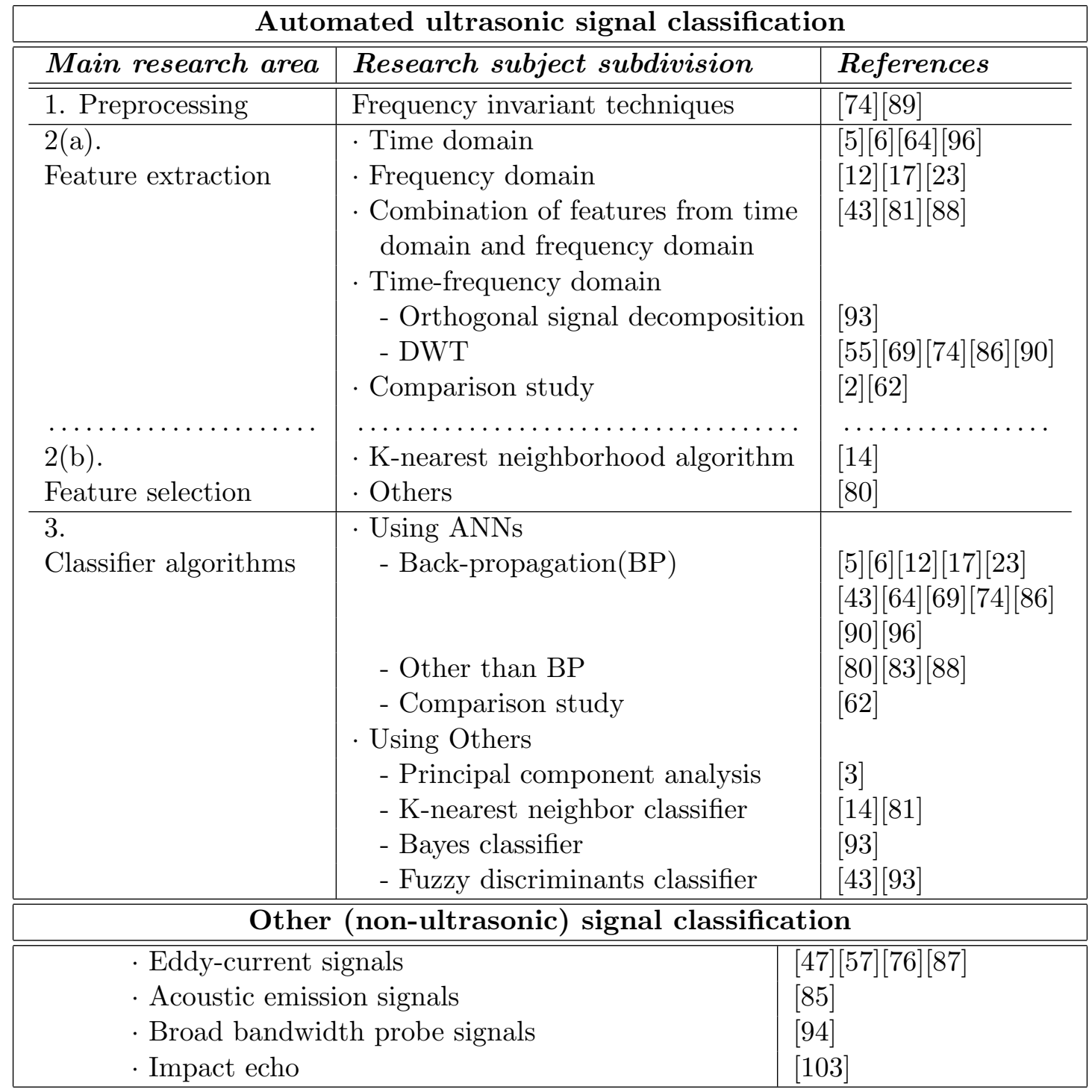


investigated the feasibility of using those features for AUSC systems. Previously proposed features include time-domain waveforms which were processed through a series of preprocessing methods [5, 6], principal components of signals [3], moments of Fourier transform of signals [12] and other informative descriptors from combinations of time-domain and frequency-domain features $[81,88,96]$.

Instead of using a set of extracted features from time and frequency domains, a whole signal section derived from the ultrasonic scans, employing a minimum of preprocessing using only Fast Fourier Transform (FFT), has been used directly as inputs into the classifier [23, 62]. More recent studies on the ultrasonic flaw classification employ Discrete Wavelet Transform (DWT) as a part of their feature extraction scheme as DWT provides considerably effective signal compression and data reduction $[69,86]$. Further details of the feature extraction schemes that can be applied to AUSC systems will be discussed in Chapter 2 .

In their quest for better sets of features for AUSC, many researchers have compared these two feature extraction schemes (FFT and DWT), classifying short signals taken from plate or pipe surfaces and distinguishing corrosive from intact ones. Most comparisons between FFT and DWT showed a superiority of DWT over FFT, for example discriminating between types of flaws (or its non-existence) [74, 78, 90]. However, in long shafts there is reason to believe that phase information is relevant as the creation of a mode-conversion has implications for the phase information. The comparative superiority of DWT over FFT as a feature extraction scheme in more complex ultrasonic signals from shafts is also analyzed and established experimentally in Chapter 3 and Chapter 4 of this thesis.

Despite the potential of using DWT for ultrasonic shaft test data analysis due to its effective time-frequency representation of non-stationary signals, the usability of DWT coefficients as a feature set has received little enthusiasm because wavelet coefficients are variant to shifts in measured time-domain signals. To overcome this time-variance problem of DWT, we need to derive a better preprocessing method for time-domain A-scan signals. We developed a new preprocessing technique which offers the consistent extraction of a segment of the signal from long shaft signals. Chapter 5 describes the experimental evaluation about the newly developed technique.

Once the feature extraction process has been completed, a suitable decision making algo- 
rithm for classification is applied to determine and classify the flaw type information. A number of supervised or unsupervised classification algorithms for AUSC systems have been proposed and they are categorized into three types: 1) Statistical classifiers such as Fisher's linear discrimination function, the minimum distance classifiers, the K-nearest classifiers or Bayes classifiers, 2) Rule-based classifiers such as fuzzy classifiers and 3) Learning-based classifiers including ANN classifiers. The feasibility of those classifiers also has been investigated through various studies comparing different classifiers [2].

Among the various learning mechanisms for ultrasonic signal classification, Artificial Neural Networks (ANNs) have gained more popularity and are considered to outperform conventional pattern recognition algorithms [62]. Many researchers use Artificial Neural Networks (ANNs) due to their ability to generate complex decision boundaries in the multidimensional feature space. This is attractive, especially in ultrasonic flaw classification, because the relationship between ultrasonic signal characteristics and their defect class is highly non-linear; thus, it is not straightforward [43, 81].

However, most research involving ANNs for analyzing ultrasonic signals has focused only on classifying short signals taken from plate or pipe surfaces $[2,90]$, where there are no side walls and no mode-converted echoes to contend with. These mode-converted echoes cause very important ultrasonic signatures to be discriminated in the testing of lengthy shafts as mentioned before. To address such a need, an initial test [23] suggested that ANNs can be used with some success to discriminate not only between the spectral signature of echoes arising from different types of reflectors such as cracks or actual features of the shaft, but also between straight compression wave echoes and shear wave mode converted echoes.

Although those initial studies to find better classification algorithms for AUSC systems are important, further studies are required to evaluate which feature extraction mechanism is best for an AUSC system for shaft testing using ANNs as its classification algorithm. The claims in previous studies from similar scenarios on the superiority of DWT coefficients to FFT coefficients as features for AUSC are subject to debate, because most previous comparison studies used only partial components (i.e. magnitudes) of the transformed signals using FFT as feature vectors for ANNs. Therefore, in order to make a fairer comparison between FFT and DWT, we formed a new set of FFT feature vectors which effectively represent both magnitudes and phases of FFT coefficients. The detailed description of the 
new presentation of FFT coefficients is described in Subsection 2.3.2, and the performance of feature extraction by FFT and by DWT is analyzed in Chapter 3 by using the resulting pattern vectors as input to training ANNs.

Though the demonstrated superiority of DWT was initially tested on ANNs as the classifier, considering the many difficulties inherent in the ANN learning paradigm (such as generalization control, overfitting and parameter tuning) we remained more conservative about DWT's predominance. In order to confirm the potential of DWT as an efficient feature extraction scheme for ultrasonic shaft test signals, we made a new comparative experiment involving Support Vector Machines (SVM) and presented the discussion of experimental design and its results in Chapter 4. The experimental results showed once again a superior performance of DWT as the feature extraction scheme in the classification of echoes from ultrasonic signals in long shafts. This confirmed the DWT as indeed superior because it provided better classification with another classifier, namely, the SVM, for which statistical properties indicate robustness in its construction, especially when a limited number of training examples are available.

While we mainly focus on finding the best single classifier model (between ANN and SVM) and determining the best selected feature extraction scheme (between FFT and DWT) for the selected single classifier model, we can also learn multiple models of the shaft test data and combine their outputs for making a final decision for classification. The motivation of this ensemble of classifiers is that FFT might reflect physical properties that are different from those that DWT conveys. We suspected that including the FFT as another informant of the decision process, even if the accuracy using DWT has shown to be superior, should improve accuracy. The diversity of techniques for generating and combining models raises the issue of which generation-combination method to choose for constructing the most effective and reliable multi-model systems for our application domain. In Chapter 6, we explore the diverse possibilities of heterogeneous and homogeneous ensembles, combination techniques, feature extraction methods and classifier types and determine guidelines for heterogeneous combinations that result in superior performance. 


\subsection{Aims and Contributions}

The background and motivation of this thesis are mentioned in previous sections in this chapter. In summary, the main research question is how to discriminate efficiently the different types of reflectors among the large volumes of digitalized ultrasonic shaft defect information. The reflectors may produce different types of reflections. We want to automate their classification into 1) those that correspond to design features of the shaft, 2) those that correspond to flaws, cracks and other defects emerging on the shaft and 3) the multiple reflections and mode-converted echoes of 1) and 2). Conventional NDT techniques, which are based on the heuristic experience-based echo-dynamic pattern identification methods, bring about costly, lengthy and error-prone analysis and thus lead to inconsistencies in results. To solve this, a variety of modern signal processing techniques and pattern recognition techniques have been investigated and verified. However, most approaches have focussed only on analyzing short signals taken from plate or pipe surfaces, which have no side walls, hence produce no mode-converted echoes.

To address such a need, industry demands new innovative NDT techniques for shaft-typed steel pieces, and furthermore, it requires novel algorithms for the analysis of large volumes of ultrasonic shaft test data. More specifically, this thesis aims to develop a more advanced, autonomous and consistent AUSC paradigm for testing shafts. The research question and the goal of this thesis are illustrated in Figure 1.4. Furthermore, Figure 1.5 highlights our comprehensive analysis and the research points corresponding to each stage of developing an AUSC system for testing shafts.

The following is a list of contributions made in this thesis.

1. Introduction of a new FFT-based feature extraction scheme (FFT_Magpha) by which both magnitude and phase components of FFT sequences are effectively represented. Through this newly developed feature extraction scheme, we can incorporate the "phase" component of FFT sequences (which can, in other schemes, usually be ignored in the process of extracting the frequency components) to the construction of the FFTbased feature vectors. The experimental comparison between this new scheme and the traditional scheme (FFT_Mag) by using them in a typical AUSC system, shows that 


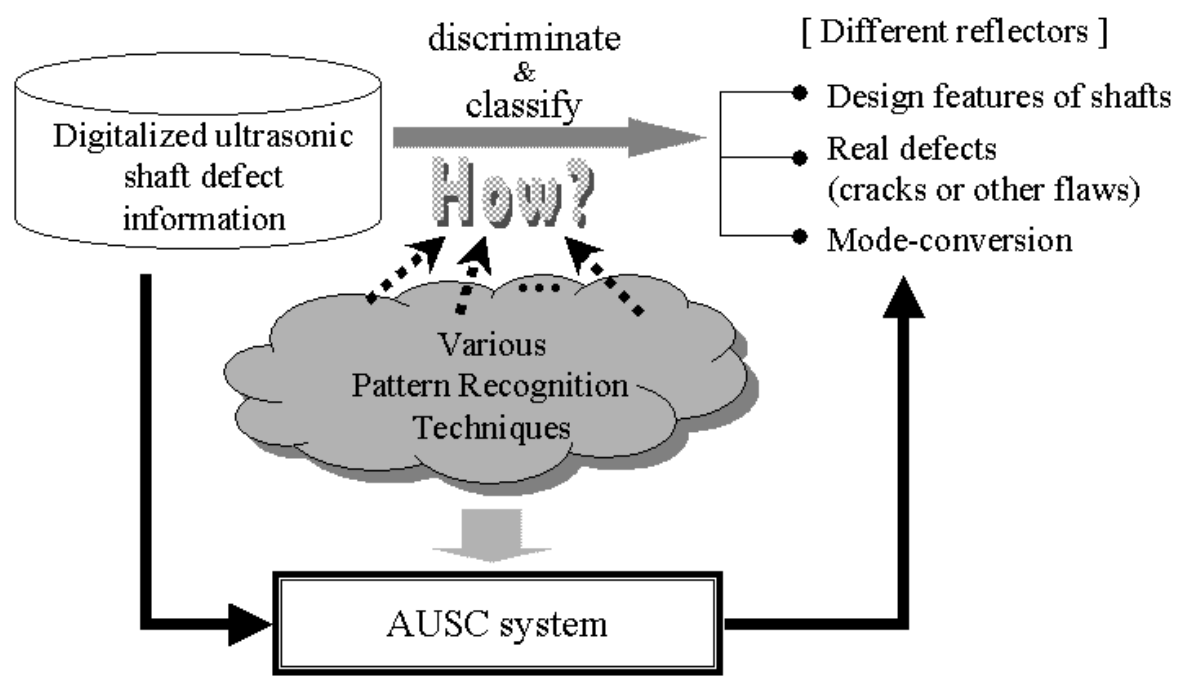

Figure 1.4: The research question and the goal of this thesis.

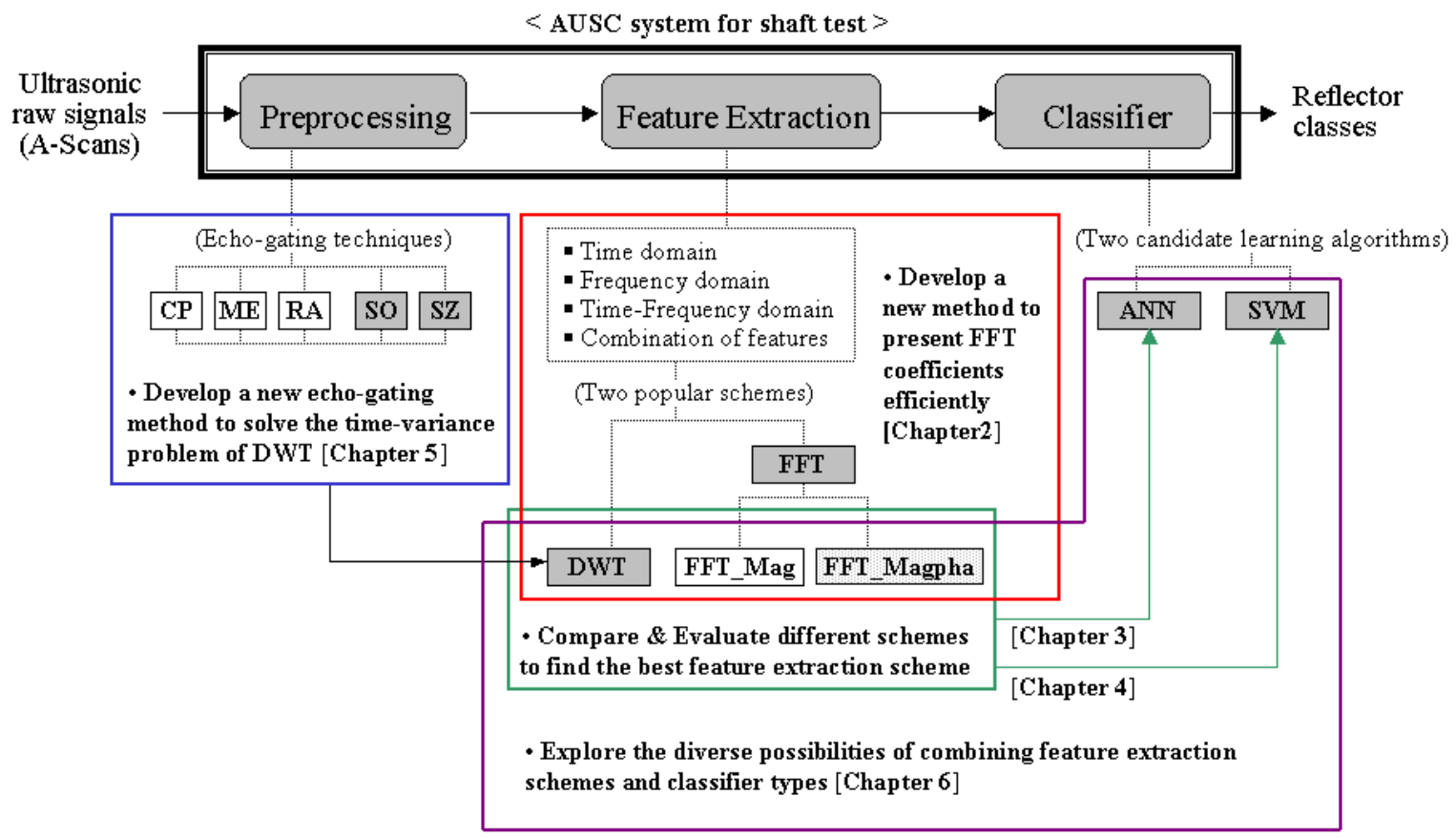

Figure 1.5: The categorization of our research aims corresponding to each stage of developing an AUSC system. 
the new scheme (FFT_Magpha) highly enhances the precision as well as improving their training and execution time.

2. Analysis of DWT as a more beneficial feature extraction scheme than FFT in an AUSC system for testing shafts. This is analysed through the experimental comparison of the classification performance using DWT, not only with the classification performance using the traditionally-used-FFT with limited feature components, but also with the newly-proposed-FFT scheme (FFT_Magpha). This extended comparison between DWT and the state-of-the-art FFT provides a more reliable and trustworthy analysis about DWT as a feature extraction scheme for our application.

3. Finding the potential of DWT as a more reliable feature extraction scheme, through the more stable classification results in different runs of cross validation tests compared to the results produced in the tests using FFT-based feature extraction scheme. This potential is especially beneficial for the practical NDT for shafts, as we can train a classifier with arbitrary training data and then use the classifier for in-field ultrasonic shaft signal tests.

4. Demonstration of the superiority of using DWT as the feature extraction scheme in the ultrasonic shaft signal classification involving not only ANN but also SVM. These results dissipate any doubt that the DWT feature extraction methodology is too far suited for ANN which has been popularly employed previously in many similar experimental scenarios.

5. Discovery of predisposition to distinguish a certain facility when specific classes of echoes are concerned with different combinations of feature extraction (FFT or DWT) and classifier (ANN or SVM), though DWT is superior to FFT and SVM is superior to ANN in terms of the overall classification accuracy. This finding leads into a hybrid classifier that will improve overall performance by giving more weight to the more trustworthy sub-classifier.

6. Introduction of a new technique to derive a preprocessing method for time-domain A-scans, which offers consistent extraction of a segment of the signal from long signals that occur in the NDT of shafts, thus solving the time-variance problems of DWT. The performance of this new echo-gating technique is compared with other previously developed methods and the experimental results imply that we can use DWT 
more efficiently as a feature extraction scheme for ultrasonic signal classification by employing this new method in the preprocessing stage.

7. Introduction of the Integrated SVM Classifier (ISVM) which is a combined classification system efficiently employing the best characteristics of each of two SVM classifiers using two feature extraction schemes, FFT and DWT. The outcomes of a classifier based on FFT are not totally dismissed in this system even though the DWT-based classifier has been shown to be superior. This property of ISVM enables us to combine classifiers considering the misclassification cost to obtain a more informative classification for its application in the field.

8. Exploration of the diverse possibilities of heterogeneous and homogeneous ensembles by combining classifiers along the dimension of feature extraction mechanism, along the dimension of combination methods and along the dimension of type of classifier. The experimental result suggests guidelines for designing an integrated multi-classifier system for shaft test data by way of selectively employing the combining structure.

\subsection{Outline of the thesis}

The remainder of this thesis is organized as follows.

AUSC systems are pattern recognition systems. As feature extraction is central to any pattern recognition problem, Chapter 2 is concerned with the extraction of informative features from ultrasonic signals. This chapter explains various ultrasonic signal features based on time-domain or frequency-domain. We discuss two top approaches; namely, FFT and DWT. We introduce FFT_Magpha, a new method of presenting FFT coefficients. In this chapter we emphasize the importance of the phase components of FFT.

Chapter 3 compares FFT and DWT as feature extraction schemes for AUSC systems in the setting of data from long shafts. This chapter is concerned with employing ANN as a learning algorithm on AUSC systems. The comparison between two feature extraction schemes (FFT and DWT) is discussed through the experiment of the classification performance using each scheme as a feature extraction mechanism in the ANN-based classifier. 
While Chapter 4 has a similar structure to Chapter 3 in terms of comparing FFT and DWT as a feature extraction scheme, this chapter is especially concerned with employing SVM as a learning algorithm. With the experimental results, this chapter analyzes not only the overall classification performance but also the classification performance for each class of echoes. This information is important to detect if any potential exists in classifying different types of echoes using different feature extraction schemes. The initial analysis leads to Chapter 6 which is concerned with the idea of combining classifiers for AUSC systems.

Chapter 5 deals with the time-variance problem when using DWT and introduces a new gating technique which is a consistent way of gating echoes in order to use DWT more efficiently as a feature extraction scheme for ultrasonic signal classification. The effectiveness of the newly developed echo-gating method is analyzed through the comparison experiment with other previous techniques.

While the previous chapters focussed on finding the best feature extraction scheme by applying different feature extraction schemes on two main different classifiers (ANN and SVM), Chapter 6 and Chapter 7 focus on learning multiple models and combining their outputs for making a final decision. Chapter 6 particularly deals with an open issue: which generation and combination method to choose for constructing the most effective and reliable multi-model systems for our application domain. It suggests guidelines for the choice of ensemble structure for ultrasonic shaft signal classification. Persuaded by the investigation presented in Chapter 6, we design a cost-sensitive combined classification system named as ISVM in Chapter 7, which is constructed by a homogeneous approach efficiently employing the strength of each of two SVM classifiers (FSVM and DSVM) using two different feature extraction schemes (FFT and DWT). Through the experimental analysis in these two chapters, we discuss whether combining the classifiers improves the classification accuracy achieved by a single classifier when each different single classifier is constructed by using a different feature extraction scheme.

We conclude in Chapter 8 with a review of the work presented in this thesis and a discussion of future work. 


\section{Chapter 2}

\section{Feature Extraction Schemes for Ultrasonic Signals}

Preprocessing of the raw signals and extracting informative features from the preprocessed signals become the important basis to solve any signal classification problem [82]. In this chapter we review various ultrasonic signal features based on time-domain or frequencydomain. We discuss mainly two top approaches, namely, FFT and DWT. Section 2.1 explains the purpose of the preprocessing and provides a simple review of various preprocessing methods suggested in previous ultrasonic related studies. Section 2.2 discusses and compares various signal features which can be extracted from time-domain and frequencydomain with the summary of related work. In Section 2.3, FFT is discussed more comprehensively as a feature extraction scheme for AUSC systems. Our interests are the two types of components of FFT coefficients, which are magnitude and phase. We emphasize the phase component of the FFT sequence by introducing a new method of presenting FFT coefficients. Similarly, DWT is discussed as another feature extraction scheme in Section 2.4. Therefore this chapter provides some preliminary concepts and methodologies related to ultrasonic signal features. 


\subsection{Preprocessing of Ultrasonic Signals}

Preprocessing of ultrasonic signals is necessary to enhance classification performance as the relationship between ultrasonic signal characteristics and flaw classes is not straightforward. The most fundamental preprocessing work is a filtering process on acquired data to minimize the effects of noise and variation in test and sample conditions such as gain and frequency settings, time delays in sampled signals, and other minor differences in material properties.

In ultrasonic NDT inspections, transducers of different center frequencies are generally used depending on the wall thickness of materials, the desired resolution and the depth and the orientation of the flaw. Thus, a certain classifier trained by a specific set of ultrasonic signals may perform well with some test signals captured under conditions similar to that specific training set, but may not show the same performance with different test signals. In this case, the classifier cannot be used in practical field inspection. Therefore, it is required that the AUSC system recognizes signals of different frequencies and interprets them in such a manner that the overall performance is independent of the frequency of operation.

To overcome this problem, several approaches were proposed in previous work $[74,89]$. They include a method using time-scaling and a method using normalization. In the first method, signals captured under different frequencies are transformed to a reference frequency signal prior to feature extraction by simply scaling the time axis of the signal, resulting in either stretching or compressing the signal [74]. Especially in the case of stretching, the number of points is then reduced to the original value by eliminating the samples at the two ends, assuming that the discarded samples from each end of the interpolated signal do not carry any significant amount of information. In the other approach, a reflected signal from a non-defected (calibration) part is set up as a reference signal, a set of features is extracted from this signal and then the features in the flaw signals are normalized by the features in this reference signal [89].

Various feature extraction techniques are then applied on the signals treated on this preprocessing stage. As mentioned in the introduction of this thesis, one of the main aims of the thesis is to find and evaluate better feature extraction schemes for the AUSC systems for testing shafts. Therefore, a consistent and regularized preprocessing scheme is applied 
for the ultrasonic signals collected for all of the experiments made and presented in this thesis. The details will be described in the corresponding sections.

\section{$2.2 \quad$ Extraction of Signal Features}

Once the ultrasonic test signals acquired in a form of digitized data are preprocessed, we need to determine features from the raw signal by the use of various digital processing techniques. This process is named "feature extraction". Since not all features that can be extracted from ultrasonic signals for a given classification problem need to be used, due to their redundancy, a further process is needed for redundancy reduction by retaining only an informative subset of them. This stage of processing is called "feature selection". The two processes - feature extraction and feature selection - are combined and will be referred to as "feature extraction" afterwards in this thesis. Thus, the extraction of discriminatory features in the signal enhances the reduction of the length of the data vector by eliminating redundancy in the signal and compressing the relevant information into a feature vector of significantly lower dimension.

Most of the signals in practice are time domain signals in their raw format. In an AUSC system, the collected signals are presented in the format of an A-Scan, which is a plot of signal voltage (amplitude) versus time for one transducer position. Even though some defect information can be obtained from the amplitude of the reflected signal, this timeamplitude presentation is not always the best representation of the signal [58, 77]. For many signals, the signal's frequency content is important and most distinguished information is hidden in the frequency components. In order to find the frequency content of a signal, the Fourier Transform (FT) is used for transforming mathematically our view of the signal from time-based to frequency-based. A raw time domain signal is broken down into constituent sinusoids of different frequencies by FT. Consequently the frequency-amplitude representation obtained by FT presents a frequency component for each frequency that exists in the signal $[28,52]$.

A lot of previous work has proposed various sets of ultrasonic features chosen from time and frequency domains and investigated the feasibility of using the features for an ultrasonic 
flaw classification system. A typical procedure of constructing a set of feature vectors based on time domain and frequency domain is summarized in Figure 2.1.

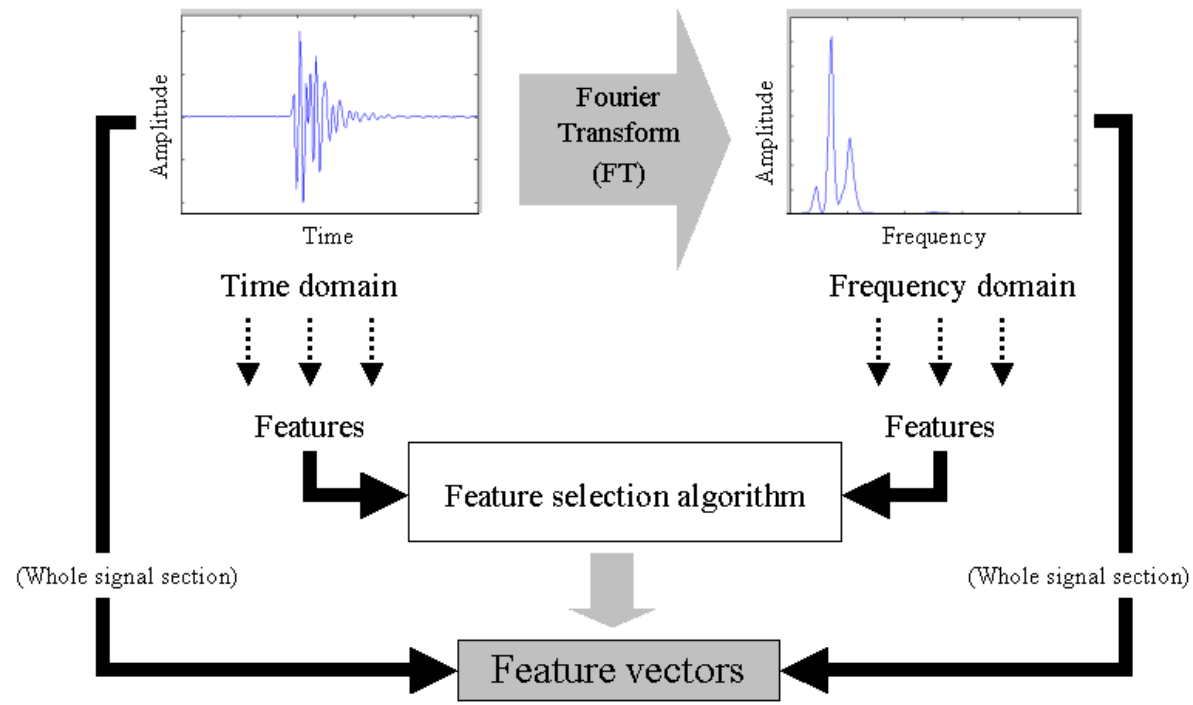

Figure 2.1: Typical feature extraction schemes based on time domain and frequency domain.

Even though the defect information has been obtained from the amplitude of the reflected signal in conventional ultrasonic testing, using only the "raw" time-domain signal data as a feature is inappropriate because a waveform changes peaks in amplitude and on time location from reading to reading (time-shift), even under ideal conditions. However, many researchers still have investigated various types of preprocessing to extract useful features from the time-domain of a signal, based on the idea that the overall waveform shape is still consistent even in every different reading [6]. The preprocessing investigated includes using a root mean square moving window and shifting back to cover time shifts, baseline subtraction to cover the variance of the background noise level and the offset of defects near to the back surface and averaging values to scale down the input feature vector. Other timedomain ultrasonic features proposed previously include principal components of signals [3] and estimates of the rectified signal envelope combined with various preprocessing methods: low-pass filtering, rectification, under-sampling, and mean-subtraction [5].

In addition to the time-domain features, various features extracted from frequency-domain have been proposed. Most frequency-domain features proposed by previous researchers are statistical parameters extracted from statistical moments of an ultrasonic frequency spec- 
trum such as mean, variance, coefficient of variance, coefficient of skewness and coefficient of kurtosis [12]. Combinations of time-domain and frequency-domain features also have been popularly used $[81,88,96]$.

This previous research has commonly traced the general guidelines of feature extraction from various domains of ultrasonic data analysis that suggest the following steps:

1. Extract as many descriptors (features) as possible from various domains.

2. Evaluate their discrimination power over the classification problem concerned.

3. Choose the best set of features.

However, there is a dilemma. Should we expand the feature set by adding new informative features for the improvement of classification performance, or decrease the number of features for the reduction of the redundancy of the feature set? Eventually, we need effective feature selection schemes to reduce this redundancy and optimize the feature set.

Various methods have been proposed and applied for the discrimination of the features $[2$, 64, 88]. Masnata and Sunseri [64] applied a Fischer linear discriminant analysis to perform a further selection from the previous extracted large set of time-domain features by maximizing discriminant information through this technique. Wilk's Lambda technique was also proposed in [2] to get a measure of data point separability in the feature domain for a specific set of descriptors and eventually to get the group of most sensitive descriptors for several classification problems. Song et al. [88] established two different feature selection schemes: "the forward selection with trial and error" and "the forward selection with criteria" to take care of the increased redundancy of the feature set properly. They also investigated the effectiveness of both approaches for the given classification problem.

On the other hand, instead of using a set of extracted features from the time and the frequency domains, an alternative idea $[23,62]$ is to use directly the whole signal section derived from the ultrasonic scans as input to the classifier. This idea is remarkable in that it demands minimum preprocessing (technically, not much feature extraction is employed at all) because it uses only the Fast Fourier Transform (FFT) technique. In particular, a comparison between two types of input data, each from the time-domain and the frequency- 
domain (using FFT) was analyzed [62]. In this case, the classification results using these two different domain features for classifying ultrasonic signals showed that frequency-domain analysis was more reliable than time-domain analysis and demanded a smaller feature size. More details of FFT and how FFT has been used as a feature extraction scheme for signal classification are presented in Section 2.3.

\subsection{Fourier Analysis and FFT}

As mentioned before, the frequency of signals is useful for analyzing signal data. The Fourier Transform (FT) decomposes a signal into a representation involving complex exponential functions of different parameters; these parameters are the so-called frequency components. FT is mathematically defined by the following two equations:

$$
\begin{aligned}
& X(f)=\int_{-\infty}^{+\infty} x(t) e^{-j 2 \pi f t} d t \\
& x(t)=\int_{-\infty}^{+\infty} X(f) e^{j 2 \pi f t} d t,
\end{aligned}
$$

where $t$ and $f$ stand for time and frequency respectively and $x$ and $X$ denote the signal at hand in time domain and in frequency domain respectively. Equation 2.1 is called the Fourier transform of $x(t)$ and Equation 2.2 is called the inverse Fourier transform of $X(f)[11,68]$.

Because a digital computer works only with discrete data, numerical computation of the Fourier transform of the signal $x(t)$ requires discrete sample values of $x(t)$, which is represented as $x(n)$. In order to deal with discrete time-series $x(n)$, the discrete Fourier transform (DFT) is used and defined by the following equation:

$$
X[k]=\sum_{n=0}^{N-1} x[n] e^{-j(2 \pi / N) k n} \quad(k=0,1,2, \ldots, N-1)
$$

whose inverse transformation is: 


$$
x[n]=\frac{1}{N} \sum_{k=0}^{N-1} X[k] e^{j(2 \pi / N) k n} \quad(n=0,1,2, \ldots, N-1) .
$$

The DFT may be executed with less computation by using a more efficient algorithm called the fast Fourier transform (FFT) [65]. These algorithms reduce the problem of calculating an N-point DFT to that of calculating many smaller-size DFTs. The only constraint in using FFT is that the input waveform must have a number of samples, each one being an integer power of two $\left(2^{n}\right)[44,63]$. Although this constraint may seem limiting, the FFT can be applied to any input through the use of zero padding.

The sequence of frequency components of a signal obtained by FFT becomes the basis for extracting the signals's frequency-domain features. A typical process for using those features for AUSC systems is to extract various features from FFT sequences, then select an optimal subset among the extracted features and finally use them for the input vector for classification. However, this feature extraction process requires a series of sub-processes for feature extraction and selection. In this thesis, we mainly focus on using the whole FFT sequence as features rather than selecting only parts of features. Section 2.3.1 describes the traditional approach for using the whole FFT sequence and Section 2.3.2 introduces a newly developed method for presenting the FFT sequence including phase information and frequency information.

\subsubsection{FFT coefficients using magnitude (FFT_Mag)}

Though the original signal sequence in time-domain consists of real numbers, the sequence of FFT of a signal is a sequence of complex numbers. Those complex numbers contain important information about the transformed sequence including its magnitude and phase. In order to use an FFT sequence practically as a feature vector, the original FFT sequence is multiplied by its complex conjugate. The multiplied sequence is no longer a sequence of complex numbers. Moreover, the last half of the multiplied sequence is the duplicate of its first half because the last half of the original FFT sequence corresponds to complex conjugates of the components in the first half of the sequence. In fact, the multiplied sequence corresponds to the power of the magnitudes of the original FFT sequence. Using 
this theory, an $n$ point long time-domain signal can be transformed to an $\frac{n}{2}$ long sequence of FFT frequency coefficients by discarding the duplicate part of the frequency coefficients (the phase coefficients are not considered in this case). These $\frac{n}{2}$ long FFT coefficients constitute frequency information and have been used popularly as FFT-based feature vectors in previous related work [23]. We name these FFT coefficients "FFT_Mag" as their physical interpretation is as the magnitude of the frequencies. With this name we will distinguish them from the variant in Section 2.3.2 where we introduce coefficients that include phase.

\subsubsection{FFT coefficients using magnitude and phase (FFT_Magpha)}

If we follow the process of the transformation in the previous section as a method to select coefficients, the phase component of the FFT sequence is excluded and only the magnitude information remains on the input-sequence for classification. In long shafts, there is reason to believe phase information is relevant, as echoes usually include a phase change. Thus, we present a new FFT-based feature extraction scheme that we call "FFT_Magpha". The proposal presents both magnitude and phase information of FFT sequences to the classifier and at the same time does not increase the dimension of the vector for classification. The main idea is to extract the magnitude component and the phase component separately from one complex FFT sequence, and rearrange them by concatenation after down-sampling to construct a final feature vector. We later show that the information loss of down-sampling is well compensated by the information brought in by the phase coefficients.

The magnitude is calculated by the same process described in Section 2.3.1. The phase component of an FFT sequence is obtained by calculating the logarithm of the original FFT sequence and then extracting only the imaginary part from it. Thus, the phase components of an FFT sequence is a vector of angles presented in radians. In order to produce smoother phase plots, we need to correct these phase angles by adding multiples of $\pm 2 \pi$ when absolute jumps between consecutive elements of the original phase sequence are greater than the default jump tolerance of $\pi$ radians. Through this calculation process, like the process of getting the magnitude component, the last half elements of the phase vector become the duplicate of its first half elements because the last half of the original FFT sequence corresponds to complex conjugates of the components in the first half of the sequence. We 
can also obtain phase sequence of length $\frac{n}{2}$ from the $n$ points in the time-domain signal by discarding the duplicated part of the sequence. The final step of the "FFT_Magpha" is to concatenate the $\frac{n}{2}$ long magnitude sequence and the $\frac{n}{2}$ long phase sequence, and this concatenated sequence becomes the final FFT coefficients. A flowchart showing the process of constructing "FFT_Mag" and "FFT_Magpha" is presented in Figure 2.2. The

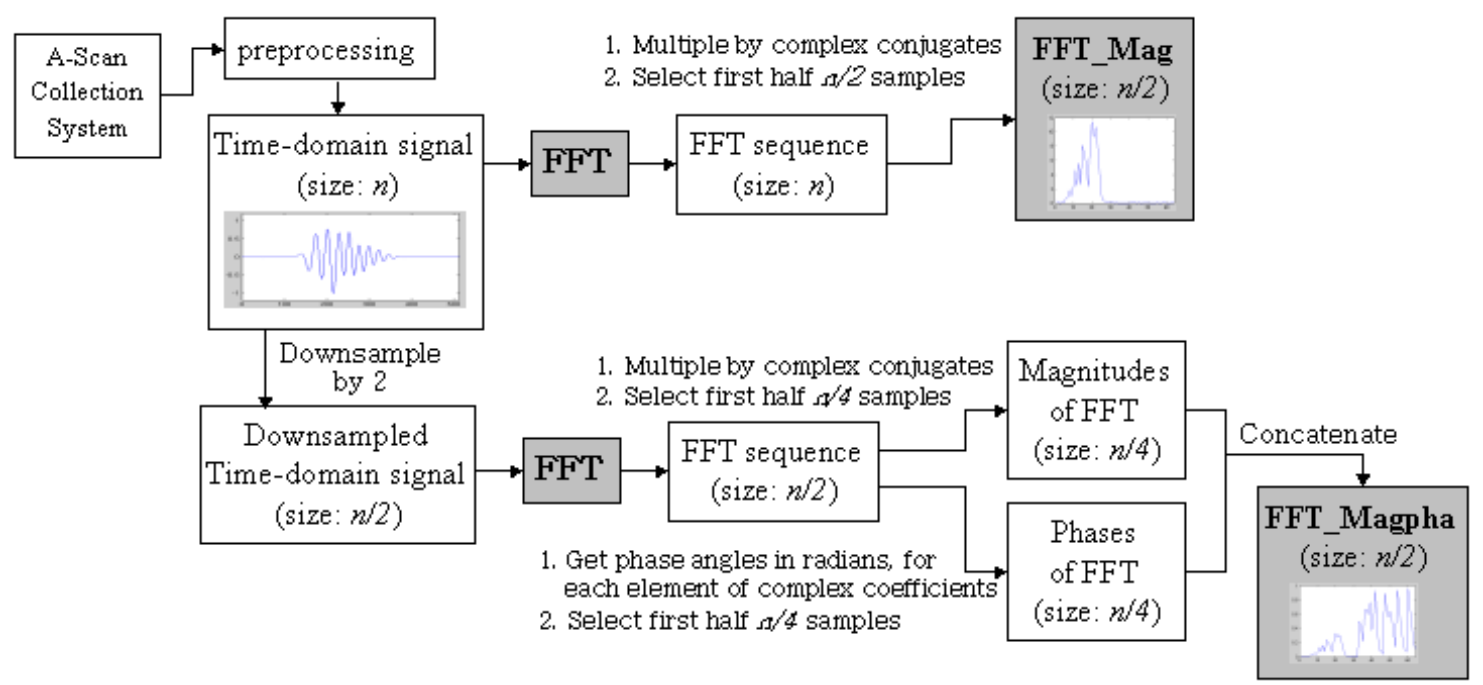

Figure 2.2: The procedure of constructing FFT_Mag and FFT_Magpha

final feature vector has the same number of elements because we apply down-sampling (by at least two) on the FFT sequence for the "FFT_Magpha" method. This adjustment of the length of the feature vectors is especially required for using them for the comparison experiment between two FFT schemes presented in Chapter 3.

It is meaningful to employ "FFT_Magpha" instead of employing "FFT_Mag" as an FFTrelated feature extraction scheme for an AUSC system. The reason is that the subject of many previous studies as well as this thesis is to investigate the feasibility of FFT as a better feature extraction scheme than other types of feature extraction schemes such as DWT. The problem is that in most previous comparison studies, only "FFT_Mag", which contains only limited components of FFT sequences, has been compared with other schemes. Therefore, it will become more fair to compare "FFT_Magpha" rather than "FFT_Mag"with other schemes like DWT. In the experiment presented in Chapter 3, the classification performances of using "FFT_Mag" and "FFT_Magpha" respectively as feature vectors to ANN 
are compared and analyzed. The result shows that "FFT_Magpha" is a more efficient FFT based feature extraction approach than "FFT_Mag". This result confirms that the phase components of FFT sequences are important information carriers and must not be ignored. From now on, we use "FFT_Magpha" as the benchmark when we refer to FFT based features.

\subsection{Wavelet Analysis and DWT}

Frequency-domain features (mainly using FFT) are generally known to surpass time-domain features in covering the problem of temporal-shift variance in the signal and in compressing the information contained in the signal into a relatively small set of descriptors. However, Fourier analysis has a serious drawback, namely, the time information is lost in transforming to frequency information. In other words, when looking at a Fourier transform of a signal, it is impossible to tell when a particular event took place. If the signal properties do not change much over time (that is, if it is what is called a stationary signal) this drawback is not very important. However, most practical ultrasonic NDT signals contain reflections from discontinuities, which result in time varying spectral characteristics of the signal. Consequently, the conventional Fourier decomposition technique is not quite appropriate to analyze the behavior of these signals.

In an effort to correct this deficiency, time-frequency representation of a signal is suggested to analyze time-localized signals with time-varying spectra, where conventional Fourier transform analysis methods are proven to be inadequate. The Short-Time Fourier Transform (STFT) is one of the time-frequency representations, and involves a technique called windowing of the signal by which the Fourier transform is adapted to analyze only a small section of the signal $[67,75]$. The STFT consequently maps a signal into a two-dimensional function of time and frequency. It is defined by Equation 2.5.

$$
\operatorname{STFT}_{X}^{(w)}\left(t^{\prime}, f\right)=\int x(t) w^{*}\left(t-t^{\prime}\right) e^{-j 2 \pi f t} d t
$$

where $x(t)$ is the time-domain signal itself, $w(t)$ is the window function, and $w^{*}$ is the 
complex conjugate of the window function $w$.

The STFT provides some information about both when and at what frequencies a signal event occurs, but we can only obtain this information with limited precision which is determined by the size of the window. While it is useful for the STFT to compromise between time and frequency information, the drawback is that once a particular size for the time window is chosen, that window size is the same for all frequencies. That is, narrow window sizes give good time resolution but poor frequency resolution while wide window sizes give good frequency resolution but poor time resolution. Most signals require a more flexible approach where we can vary the window size to determine more accurately either time or frequency.

In order to overcome the dilemma of the resolution problem of the STFT, the Wavelet Transform (WT) was developed and applied in the area of signal processing. WT adapts a windowing technique with variable-sized regions, in which the use of long time intervals is allowed where more precise low-frequency information is required and shorter regions are used where high-frequency information is required [21, 27]. Figure 2.3 illustrates the common formats for displaying a signal and contrasts the WT view with the time-based view, the frequency-based view and the STFT view.

More precisely, while Fourier analysis consists of breaking up a signal into sine waves of various frequencies, wavelet analysis decomposes a signal into shifted and scaled versions of the original (or mother) wavelet. Here a wavelet is a waveform of effectively limited duration that has an average value of zero, while sinusoids, which are the basis of Fourier analysis, do not have limited duration and extend from minus to plus infinity. Due to this theory, wavelets tend to be irregular and asymmetric while sinusoids are smooth and predictable. The WT in a continuous time scenario is defined in the following equation:

$$
W T_{x}^{\psi}(\tau, s)=\Psi_{x}^{\psi}(\tau, s)=\frac{1}{\sqrt{|s|}} \int x(t) \psi^{*}\left(\frac{t-\tau}{s}\right) d t
$$

where the transformed signal $W T_{x}^{\psi}(\tau, s)$ is a function of two variables, $\tau$ and $s$, representing translation and scale parameters, respectively, and $\psi(t)$ is the transforming function called the mother wavelet. The wavelet transform represents the correlation between the signal 


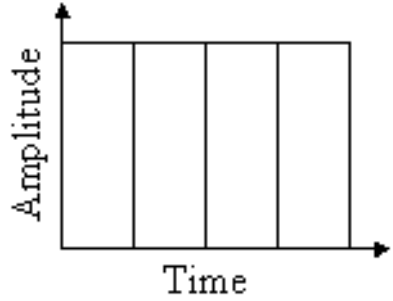

Time Domain

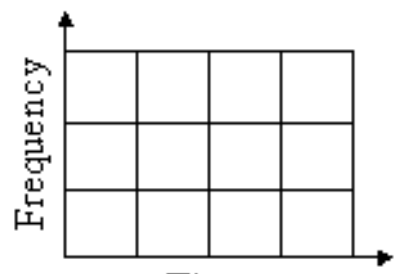

Time

STFT

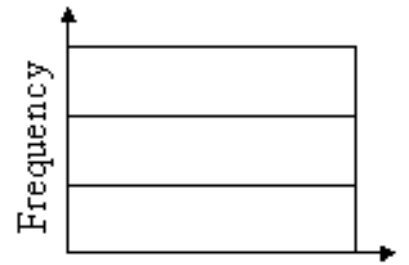

Amplitude

Frequency Domain

(Fourier)

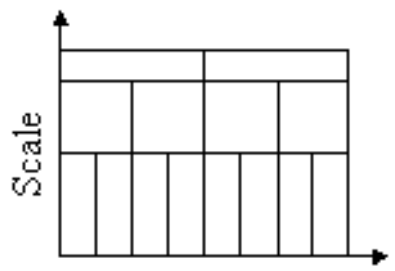

Time

Figure 2.3: Four different views of a signal depending on different domains: time-domain, frequency-domain, STFT and Wavelet analysis.

$x(t)$ and scaled versions of a prototype function (mother wavelet). The scaling of the prototype function involves contraction and dilation of the signal, and the translation involves shifting this function along the time axis.

One major advantage afforded by the WT is the ability to perform local analysis, that is, to analyze a localized area of a large signal. Based on this advantage, wavelet analysis is capable of revealing aspects of data like trends, breakdown points, discontinuities in higher derivatives, and self-similarity. Furthermore, wavelet analysis can often compress or denoise a signal without appreciable degradation. These advantages of the wavelet method also have been shown practically in the extraction of information of flaws from ultrasonic Lamb waves where the received signal is naturally noisy $[55,79]$.

Especially, Discrete Wavelet Transform (DWT) has been widely used in the ultrasonic signal analysis as a fast algorithm to obtain the wavelet transform of a discrete time signal $[35,50]$. This method comes from the idea of calculating wavelet coefficients at only a subset of scales and positions rather than at every possible scale. Moreover, DWT employs a much more 
efficient method. This is by so called dyadic scales and positions arranged as powers of two. An efficient way to implement this scheme using filters was developed by Mallat [60] and the algorithm is known as a two-channel subband coder. Figure 2.4 illustrates the one dimensional DWT decomposition algorithms. More details of this algorithm follow next in Section 2.4.1

\subsubsection{DWT coefficients}

The DWT analyzes the signal by decomposing it into its coarse approximation and its detailed information, which is accomplished by using successive highpass and lowpass filtering operations in the frequency domain. The original signal $x[n]$ is first passed through a half-band highpass filter $g[n]$ and a lowpass filter $h[n]$, where $g[n]$ and $h[n]$ are quadrature mirror filters (QMF) of each other. Various filters have been developed to be used in DWT computation, and among them Daubechies wavelets have found widespread use [26]. After the filtering, half of the samples of the two output signals are discarded by downsampling, because the signals now have a bandwidth of $\pi / 2$ radians instead of $\pi$. This constitutes one level of decomposition and is expressed mathematically as:

$$
\begin{aligned}
y_{\text {high }}[k] & =\sum_{n} x[n] \cdot g[2 k-n], \\
y_{\text {low }}[k] & =\sum_{n} x[n] \cdot h[2 k-n],
\end{aligned}
$$

where $y_{\text {high }}[k]$ and $y_{\text {low }}[k]$ are the outputs of the highpass and lowpass filters, respectively, after downsampling by 2 . The above procedure is repeated for further decomposition of the lowpass filtered signals. The highpass filtered signals constitute the first DWT coefficients. The DWT coefficients at different levels are concatenated, starting with the last level (coarsest) coefficients. This decomposition algorithm is illustrated in Figure 2.4. For a signal consisting of 256 samples, for example, the first level (highest resolution) contains 128 samples, the second level contains 64 , the third level contains 32 , and so on.

The DWT also provides a very effective signal compression and data reduction scheme. Because the energies of most signals are concentrated in a certain frequency band, all other frequencies are represented by very low amplitudes in the transform's domain, and can be discarded with little or no loss of information. In this respect, DWT not only can be 


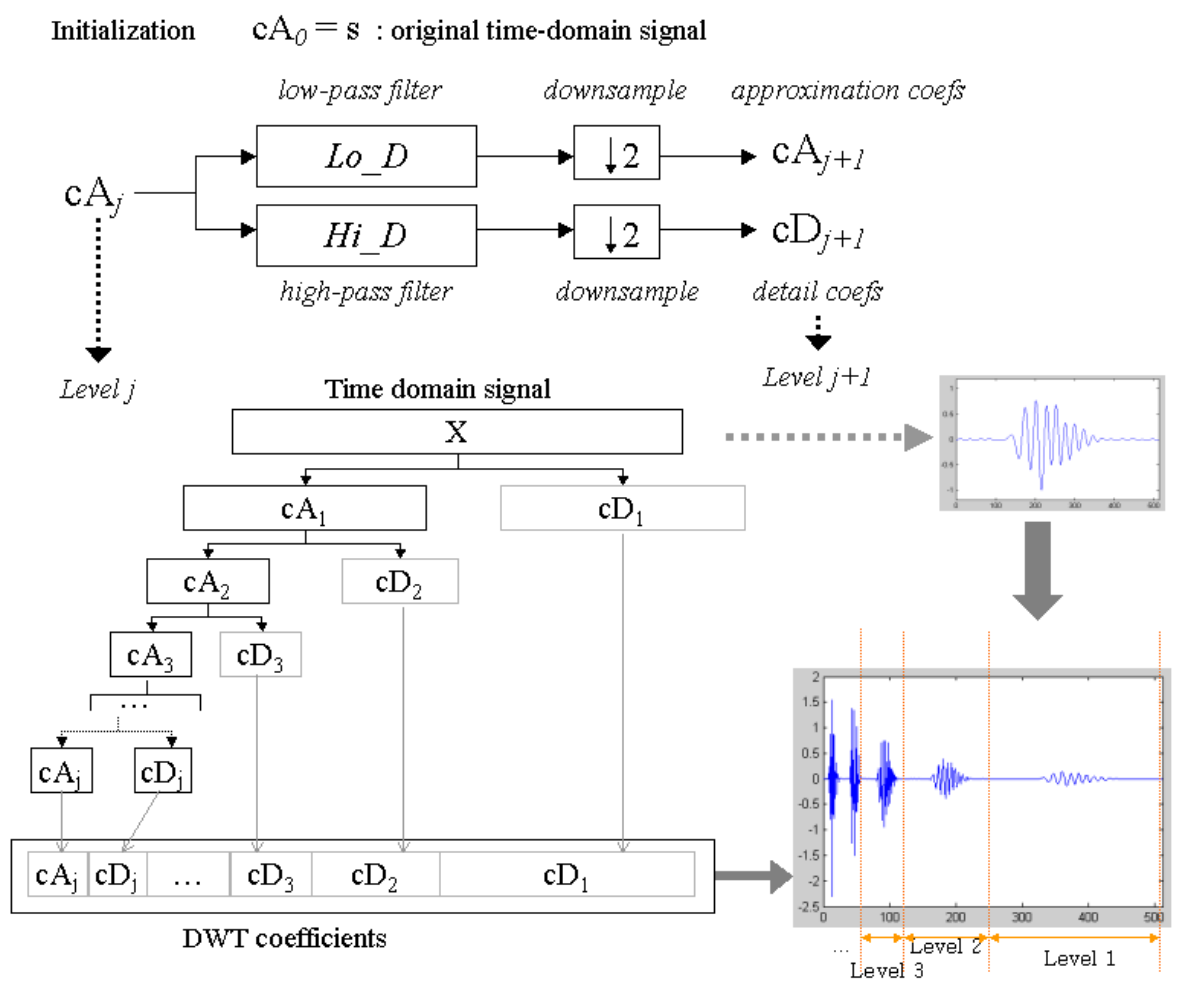

Figure 2.4: One-dimensional DWT decomposition algorithm. 
used as an efficient feature extraction scheme for the signal classification, but also provides significant data reduction, thereby reducing the computational burden considerably. Furthermore, this data reduction also may improve the classification accuracy because the excluded samples are most likely to correspond to noise.

\subsubsection{DWT as a feature extraction scheme}

Most of the recent work on ultrasonic flaw classification employs the DWT as part of its feature extraction scheme. There have been proposals of a method of ultrasonic signal analysis that is based on a selection process of coefficients provided by the DWT. This method ultimately shows that DWT provides a very effective signal compression and data reduction scheme.

Simone et al. presented three different feature extraction techniques using the Discrete Gabor Transform (DGT), coefficients of DWT and the clustered DWT (first, we cluster the wavelet coefficients using a clustering algorithm and then we use the energy of each cluster as a feature) for the classification of ultrasonic NDE signals acquired from nuclear power plants [86]. Artificial Neural Networks (ANN) was trained and tested using the three different feature extraction techniques above, and the results, measured by classification accuracy, demonstrate the usefulness of the clustered DWT method over the two other well known techniques, DGT and DWT. Obaidat et al., in their experiment, used a raw A-scan and compared two classification results [69]. They conclude that the DWT provides not only a feature extraction scheme but also significant data reduction, thereby reducing the computational complexity. The superiority of the DWT to FFT as a feature extraction scheme became evident in other research also [74], where different preprocessing techniques using coefficients of DWT and FFT are compared and performance is measured by ANN classification accuracy. Others [78, 90] also demonstrate the feasibility of DWT as a suitable feature extraction scheme for the analysis of ultrasonic NDT signals.

However, most previous comparison studies of different feature extraction schemes (especially between the use of FFT coefficients and the use of DWT coefficients) have focussed only on classifying short ultrasonic signals taken from plate or pipe surfaces where there are no side walls and no mode-converted echoes to contend with. These mode-converted echoes 
cause very important ultrasonic signature to be discriminated in the testing of lengthy shafts as mentioned in the introduction of this thesis. To address such a need, this thesis aims to report the investigation into whether DWT can outperform FFT at extracting features in ultrasonic signals from shafts and support the claim in previous studies from similar scenarios on the superiority of DWT coefficients over FFT coefficients as features for AUSC systems. In Chapter 3 we report on the comparison between DWT and FFT through the classification performance of ANN. We report on the use of SVM in Chapter 4.

Even though DWT coefficients are considered as useful features for input into classifiers due to their effective time-frequency representation of not-stationary signals, DWT exhibits a time-variance problem that has resulted in reservations about its wide acceptance. In other words, DWT coefficients can display large variance depending on how flaw signals are captured and gated from the original A-scans. We deal with this time-variance problem of DWT coefficients in Chapter 5 with more detail and introduce a new technique to derive a preprocessing method for time-domain signals, by which DWT coefficients can be used as a feature extraction scheme more reliably. 


\section{Chapter 3}

\section{FFT vs DWT using ANN Classifiers}

\subsection{Introduction}

Like all applications dealing with pattern recognition and classification matters, the main concern within the AUSC research community is the extraction of the set of features so that classification is efficient and accurate. Among the various ultrasonic feature extraction schemes previously proposed, the FFT scheme remains very well known and useful for extracting frequency-domain signal features [23, 62]. More recent studies on ultrasonic flaw classification employ the DWT scheme as part of their feature extraction because the DWT provides effective signal compression as well as time-frequency presentation $[69,86]$.

We chose features in order to apply classifiers that sort out signals. There are a number of classification algorithms. Researchers have investigated their feasibility through various studies that compare different characteristics of the classifiers [2]. The relationship between ultrasonic signal characteristics and their defect class is not straightforward. Therefore, we cannot expect a simple function to properly model the classification; for example, a linear discriminant would certainly be inappropriate. Many researchers of AUSC-related topics consider Artificial Neural Networks (ANNs) attractive because they constitute a model with 
the ability to make non-linear decision boundaries. Now, ANNs use the back-propagation algorithms in order to heuristically optimize their performance, starting from a random configuration of weights. This rather efficient method to train the network has resulted in classifiers that outperform other conventional pattern recognition algorithms (and even in some cases other approaches to apply ANNs for ultrasonic signal classification) [62]. The popularity of ANNs prompts researchers to seek better sets of features for AUSC. For example, fewer features directly imply a smaller neural network and thus fewer weights, reducing the possibility of over-fitting or of back-propagation converging to a local optima of the Total Squared Error. Therefore, much effort has been invested in comparing several feature extraction techniques and then evaluating the classification performance of a number of variants of ANN-based classifiers. The related previous studies are summarised in Section 3.2. Typically, the experiment consists of training with the new feature set and comparing it with another feature set. In the same line, the community has performed studies that compare the FFT and the DWT in order to identify their ability and potentially their superiority as feature extraction schemes. At least for short signals, most results indicate a superiority of the DWT scheme over the FFT scheme [74, 78, 90].

However, most research involving ANNs for analyzing ultrasonic signals has focused only on classifying short signals taken from plate or pipe surfaces and distinguishing corrosive surfaces from intact ones [2][90]. In all cases, there were no sidewalls and no mode-converted echoes to contend with. These mode-converted echoes cause very important ultrasonic signature to be discriminated in the testing of lengthy shafts as mentioned before. To address such a need, an initial test [23] suggested that ANNs can be used with some success to discriminate not only between the spectral signature of echoes arising from different types of reflectors such as cracks or actual features of the shaft, but also between straight compression wave echoes and shear wave mode converted echoes.

In this chapter, we report our investigation into whether DWT can outperform FFT at extracting features in ultrasonic signals from shafts. The claims in previous studies from similar scenarios on the superiority of DWT coefficients over FFT coefficients as features for the ultrasonic signal classification is subject to debate. The reason is that most previous comparison studies used only the magnitude component of the transformed signals using FFT and their phase components were naturally excluded through the process of using FFT 
coefficients as feature vectors for ANN. Therefore, in order to make a fairer comparison between FFT and DWT, we extracted phase components as well as magnitude components of FFT coefficients and formed a new set of FFT feature vectors which effectively represent both magnitudes and phases of FFT coefficients. The classifier will be ANNs to discriminate the different types of echoes. While the ANN architecture and parameters will be the same, we investigate whether faster training, better accuracy or more consistency is possible if the pattern vectors are obtained using DWT rather than FFT. That is, we analyze the performance of feature extraction by FFT and by DWT using the resulting pattern vectors as input to training ANNs.

The rest of this chapter is organized as follows. Section 3.2 briefly describes how ANNs have been previously used for AUSC-related studies. Section 3.3 explains the overall procedure of our experiment and details the steps involved in the experiment. That is, it describes the procedure of preprocessing and constructing a database for our experiment, and applying ANNs tests. Section 3.4 analyzes the experimental results and compares and discusses the performance of the FFT and the DWT as feature extraction schemes. Conclusions of these experiments appear in Section 3.5.

\subsection{Related Work}

Due to the ability to generate complex decision boundaries in the multidimensional feature space, ANNs are known to be well suited when the relationship between the input space and the output space is highly nonlinear or unknown [81]. Therefore, many researchers have been interested in employing ANNs for ultrasonic flaw classification because of the fact that the relationship between ultrasonic signal characteristics and defect class producing the signal is not straightforward [43]. R. Polikar et al. compared the performance of the ANN classifications with that obtained using conventional clustering techniques such as the K-means clustering algorithm [74]. Through this comparison, ANNs show superior classification performance in the case of complex feature spaces.

In many practical applications, the most popularly used paradigm of ANNs is the multilayered neural network using a back-propagation algorithm. A number of previous stud- 
ies $[5,12,16,64]$ showed the success of a multi-layered neural network for generating the nonlinear decision surfaces partitioning the feature space appropriately for classifying signals. Also, F.W. Margrave et al. compared and evaluated three different types of ANN architectures: multi-layered perceptron, Kohonen network and Learning Vector Quantisation (LVQ) network, for the classification of ultrasonic defects, and the result shows that the multi-layered perceptron architecture using the back-propagation training algorithm performs most reliably in both the time-domain and the frequency-domain [62].

On the other hand, the multi-layered neural network using back-propagation algorithm is problematic due to the trial-and-error determination of network architectures, lengthy training time and opaqueness of the decision making process to the users. Some alternatives using non-backpropagation ANN-based techniques are proposed in several previous studies $[6,43]$. W. E. Bond et al. applied two different ANN algorithm; the backprogapation algorithm and a newly-developed supervised ANN algorithm SMART2 for the ultrasonic flaw classification of aircraft parts, and compared their classification performance [6]. The results there indicated once more a considerably longer training time required for the backprogapation algorithm and proved that SMART2 was useful for evaluating and understanding the data. In addition, a combination of neural networks and fuzzy-logic for different aspects of ultrasonic data analysis has been proposed [43]. Here, a multi-layer perceptron is used for defect classification while fuzzy-logic is used for acceptance criteria for ultrasonic weld evaluation.

The Probabilistic Neural Network (PNN) [91] is a representative alternative as it has all the advantages of neural networks while excluding the typical disadvantages of back-propagation neural networks $[83,88]$. Given the fact that the architecture of a PNN can be directly determined by the provided flaw classification problem, the training of a PNN classifier can be completed instantaneously [105]. These researches also suggested applying a Bayes decision strategy to make the classification performance of PNNs consistent. B. Sadoun also supported PNN as a flaw classifier in his study and compared PNN with various other ANN paradigms including backprogapation networks, radial basis function network, general regression neural network and LVQ network [80].

Even though PNN has advantages as an alternative paradigm of ANNs to the multi layered neural networks, PNN has drawbacks which are commonly admitted [33, 48, 105]. The first 
disadvantage of PNN is that it requires higher memory demands during the execution, thus the execution of the trained network for applying new test data becomes slow. The other drawback is that its efficiency depends strongly on its initial training data. It means that PNN need to be trained with the correct proper data in order to achieve a good efficiency. Both of those two main disadvantages of PNN cannot be simply ignored when we consider PNN as the classifier for the shaft test data. The main application area of this study, which is developing an efficient classifier for ultrasonic shaft signals, is NDT (non-destructive test) for shaft inspection without disassembling shafts or disrupting their operation. Therefore, the inspection using this application mostly occurs in industrial fields using a portable in-situ testing system where the trained classifier is installed. For the purpose of this application, the slow execution of PNN classifiers, caused by high memory demands, will be problematic. Also, as mentioned in Section 1.2, the main issue in classifying ultrasonic shaft signals is that there are mode-converted echoes, thus A-scan signals taken from shafts are more complicated and unexpected than A-scans from other machine parts producing shorter and simpler signals. Consequently, it is hard to gain the clear representative data as the initial training data for the classifier.

Though the various architecture and paradigms of ANN have been proposed for ultrasonic flaw classification before, most research involving ANNs for analysing ultrasonic signals has focussed only on classifying short signals, where there are no mode-converted echoes to contend with. These mode-converted echoes are the most important signature to be discriminated in the inspection of lengthy shafts. There was only one previous analysis so far which treated this mode-conversion problem for shaft test data [23] and it suggested that ANNs using back-propagation algorithm can be used with some success to discriminate those special types of echoes. The selection of ANN architecture and paradigms that we used in this thesis is much inspired by that initial study.

\subsection{Experiment}

\subsubsection{Overall Process}

The experimental setting consists of three main processes. 
The first process is data collection. We acquired A-scan signals from various shafts with various lengths and characteristics such as non-cylindrical shapes or having holes and/or gears. To construct the database for our experiment, we captured signal echoes from the whole range of ultrasonic A-scan signals, segmented them in a regular size and normalized them. In order to build training data sets, we also recorded whether the signal originated from a crack or not and whether it was a primary or secondary (mode-converted) echo. We used $S H A F T E S T^{T M 1}$ as a tool to capture these A-scans and build up an initial database of required echoes.

Once we structured an initial database, we could start on the second process. We mapped the database vectors into their corresponding feature domain vectors. We used three different feature extraction techniques. First, FFT with magnitude component. We named this FFT_Mag. Second, FFT with magnitude and phase components together. We called this FFT_Magpha. The third feature extraction technique applied is DWT. These three techniques extract more informative features than simply using directly the signal amplitude as the vector for classification. Through the three feature extraction schemes, we obtained three different sets of training data.

Finally, the third step was training different ANN models using the batch backpropagation algorithm separately for those three different training sets. We validated the classification performance with ten repetitions of cross-validation. Figure 3.1 summarizes the whole procedure of our experiment as a flow chart. A more detailed description is presented in the following subsections.

\subsubsection{Preprocessing and Feature Extraction}

We collected data from eight different shafts ranging between $100 \mathrm{~mm}$ and $1300 \mathrm{~mm}$ long. Five of these shafts contained cracks and the rest were clean shafts. Mode-converted echoes were captured from these shafts, except for one $100 \mathrm{~mm}$ calibration block which did not produce mode-converted echoes because of its geometry. ${ }^{2}$

\footnotetext{
${ }^{1}$ This is a trade mark of CCI Pope.

${ }^{2}$ For this size shaft $(75 \mathrm{~mm}$ diameter $\times 100 \mathrm{~mm}$ length $)$, there is no geometric possibility for the shear waves to reflect and convert back to compression waves before they are simply absorbed, because the shear waves are generated at $33^{\circ}$.
} 


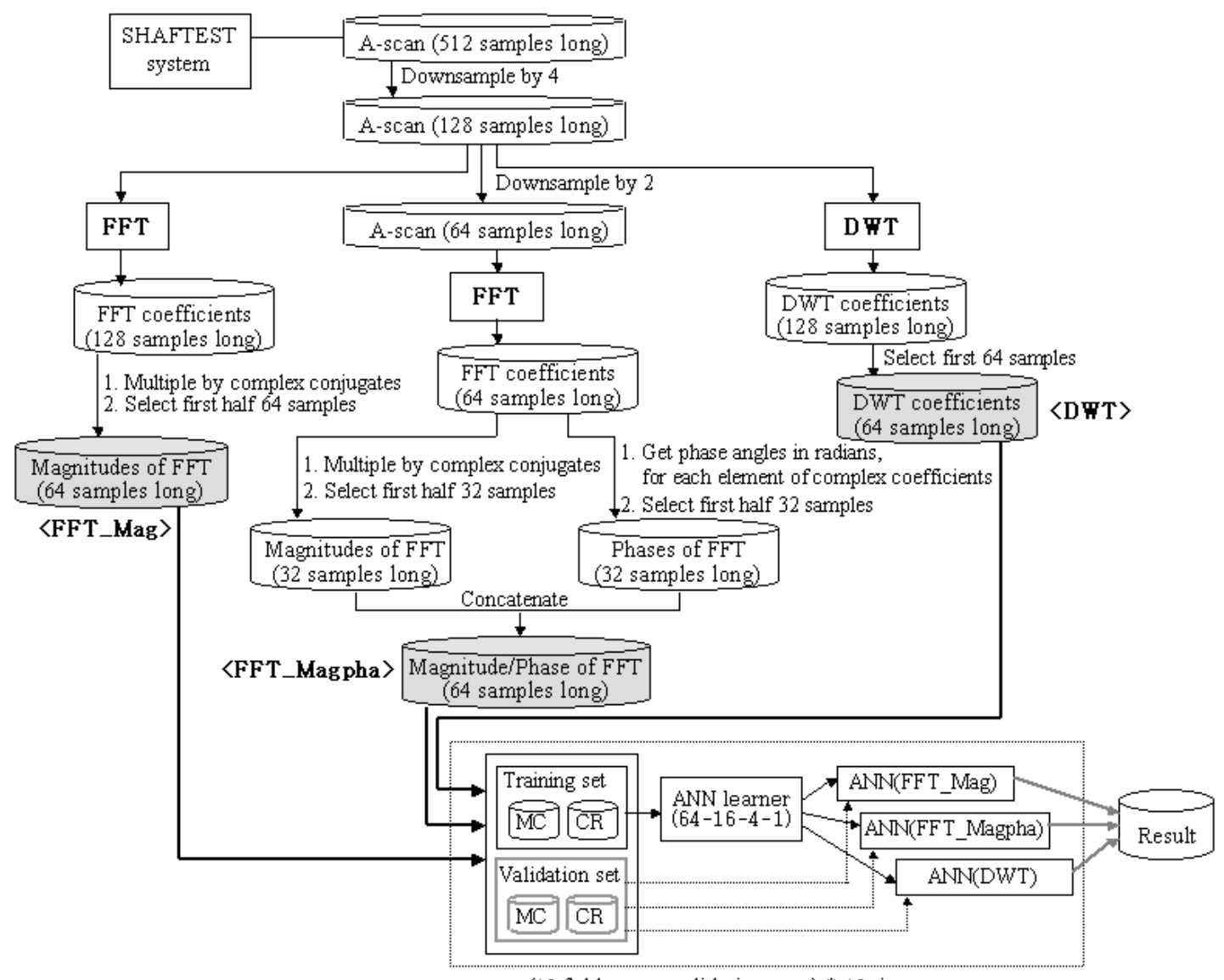

(10 folds cross validation tests) $* 10$ times

Figure 3.1: An overall procedure diagram for the experiment for ANNs classification. 
More than 1000 signals, each consisting of a segment of 512 data points, were acquired from these shafts at a $100 \mathrm{MHz}$ sampling rate using a probe at $5 \mathrm{MHz}$ frequency and normalized in amplitude to a maximum of 1 in order to avoid the variance in gain setting parameters. Whether the echoes were caused by $\operatorname{CRack}(\mathrm{CR})$, Mode-Conversion(MC) or Backwall Reflection(BR), was also recorded. Among these three causes of echoes, BR is considered to be more easily distinguished than the other two factors CR or MC [23]. Practically, one of the issues of concern in ultrasonic shaft inspection is that the signal echoes caused by CR can be confused by fainted echoes caused by MC and vice versa. Therefore, in our experiments, different feature extraction schemes are compared by the classification performance in classifying the received signal echoes just as those from CR or those from MC. For this purpose, each 60 signals were chosen randomly from CR data and MC data among the sample pool for the experiment. Thus, a data set with 120 time-domain signals in total became the basis for further processing.

The next step was converting the time domain data into different domain feature sets which would later become the input to the ANNs. For our purpose, the original data was converted into the frequency domain using the FFT and also into the time-frequency domain using the DWT. Chapter 2 presented the concepts and the methods associated with the FFT and the DWT. We down-sampled the original time domain data to reduce the dimensions of the input vector for the ANNs. We took 128 points before conversion by the FFT or the DWT. As explained in Chapter 2, the sequence resulting from the FFT of a signal is a sequence of complex numbers representing the magnitude and phase of the transformed sequence. As the last half of the sequence of FFT corresponds to complex conjugates of the components in the first half of the sequence, the FFT of 128-points-long signal yielded 64 magnitude components and 64 phase components. These 64 magnitude components of 120 FFT signals with their known classification ( $\mathrm{CR}$ or $\mathrm{MC}$ ) became one of the three different feature sets for our experiments and we named it "FFT_Mag" as mentioned before. Correspondingly, we converted the 128-data-point-long signal by the DWT and also compressed it to 64 samples by discarding the last 64 coefficients which do not contain much information but mainly noise. These converted signals named "DWT" were also stored as another feature set. For our experiment, we applied Daubechies wavelets [26] for filtering. The other feature set named "FFT_Magpha" comprises both magnitude components and phase components of FFT sequences. In order to make the dimension of this feature set exactly the same 
as the two other feature sets, we down-sampled the original 512-point-long (time domain) data into 64 points and then transformed it using the FFT. We computed 32 magnitude components and 32 phase components separately from the 64 complex elements of the FFT and concatenated them to make 64 dimensional feature vectors.

\subsubsection{ANN Classification}

The construction of the neural networks and training was performed using the Stuttgart Neural Network Simulator (SNNS) [106]. This simulator comes with a batch job mode that can be programmed to perform the task automatically and record the results. This greatly simplifies the lengthy task.

Before starting the experiment, the training was put to the test in order to determine suitable network parameters and a training algorithm (between two backpropagation algorithms provided by SNNS: standard backpropagation and batch backpropagation). Some variant algorithms to standard back propagation, such as the momentum backpropagation, introduce extra parameters, such as the momentum term which helps prevent the network from getting trapped in local minima area and converging to a zero error. Other tools include introducing noise to the input to help generalize the network performance, but thie seemed to have no effect on training and performance, perhaps due to the fact that there is already much variance and noise in the sample sets. Finally it was determined that the batch backpropagation method was the most stable in training the sample data. This training method involves looking at all the training data before making any network adjustments, and repeating this until the network error is small. Standard backpropagation makes network adjustments after each input sample is examined. Training still occurred, but in this case was much more erratic.

Various test runs were conducted using different parameters to roughly deduce their effect on training ability and training times. Increasing the learning parameter seemed to affect the rate at which the networks trained, although at the expense of training stability. A value of 0.2 seemed to provide reliable and stable training and so was chosen to be the value for all the networks tested in the experiment. The tolerance factor is another parameter we need to select to specify the maximum difference between a teaching value and the output value 
of the network. We set the threshold to 0.5 which is a default provided by the simulator. We also selected the topological order as the update mode of the network.

We also did a number of pre-experimental tests in order to select the number of hidden layers and the size of the hidden layer. As we constructed 64 dimensional feature vectors, a number of network architectures with 64 input nodes were selected as candidates. They were 64-64-1, 64-32-1, 64-16-1, 64-32-16-1, 64-16-8-1 and 64-16-4-1, representing the number of input, hidden (two numbers in the case of two hidden layers), and output units. The training of these candidate networks was put to the test and we finally chose 64-16-4-1 structure for our experiments as it was comparatively better in terms of the performance result and the slow convergence to overfitting.

Once we determined the architecture and parameters of ANNs, we shuffled the input samples (preprocessed in a way described in Section 3.3.2) and randomly divided them up into 10 sets. In turn, 9 of these were used to train the network, and the remaining set was used to validate the network. This was repeated with all ten possible combinations and furthermore, the process was repeated 10 times to get an average of the networks' training ability by assigning 10 randomly different initial weights to the network. This 10 -fold cross-validation test was carried out on three ANNs, which are trained by three feature sets: FFT_Mag, FFT_Magpha and DWT. The performance of the three different schemes can be compared using the result of each cross-validation test.

For the efficient training process, we set the Mean Square Error (MSE) limit to 0.001 for stopping the training process. We also set the epoch limit to 200000 in case the training failed to converge. To overcome the overfitting problem during the process of training ANNs, the error over the validation data were monitored and forced the training to stop on the epoch that produced the lowest error over the validation data. We plotted the MSE both over the training data and over the validation data in every training process, in order to monitor how the MSE varies with the number of epochs and to decide when to stop the training process before overfitting.

Through the SNNS Batchman job we programmed, the phaseal results during the process of training ANNs were recorded as a log in every 100 epochs for each test run. Simultaneously, we also monitored the variation of MSE through the plotted graph over the training data 
and the validation data for each test run. In order to decide the moment of overfitting more reasonably, we monitored the graphs of MSE during the whole period of training (under the limit of 200000 epochs as maximum) even when we already found the first moment of overfitting. Because it is not always obvious when the lowest error on the validation set has been reached, this comprehensive monitoring method helps to avoid the mistaken conclusion that the network has reached its lowest validation set error [66]. Once we decided the moment of the lowest error on the validation set, we looked up the results log, found the accuracy on the epoch just before the overfitting started and then considered it as the accuracy of the ANN model on that test run.

\subsection{Results and Analysis}

To test the performance of each feature extraction scheme, we recorded three result sets. Each result set corresponds to applying FFT_Mag, FFT_Magpha and DWT, and it is derived from each of the ten times of 10-fold cross validation tests (a total of one hundred tests for each network). The derived information consists of the following indicators.

1. The percentage of correct classification over the validation set.

2. The number of epochs required to train to the given error rate or to the lowest validation error rate before overfitting happens.

Table 3.1 lists the result values for each cross validation test set. The presented values for each test (from the 1st row to the 10th row of Table 3.1) are the averages calculated for ten runs of test by setting up different initial weights on ANNs. An overall average is also calculated and put on the 11th row of Table 3.1. We also calculated the corresponding standard deviation divided by this mean value (relative standard deviation) both over cross validation test sets and over different weighting runs for each test sets. They are presented on this table as RSD_1 and RSD_2.

The visual comparison between the classification performance of using three different feature extraction schemes is offered by Figure 3.2, which shows histograms presenting relative values of the results shown in Table 3.1. 
Table 3.1: Accuracy and number of required epochs for three different feature extraction schemes: FFT_Mag vs FFT_Magpha vs DWT.

\begin{tabular}{|c|c|c|c|c|c|c|}
\cline { 2 - 7 } & \multicolumn{2}{c|}{ FFT_Mag } & \multicolumn{2}{c|}{ FFT_MagPha } & \multicolumn{2}{c|}{ DWT } \\
\cline { 2 - 7 } & $\begin{array}{c}\text { Accuracy } \\
\text { (\%) }\end{array}$ & Epoch & $\begin{array}{c}\text { Accuracy } \\
\text { (\%) }\end{array}$ & Epoch & $\begin{array}{c}\text { Accuracy } \\
\text { (\%) }\end{array}$ & Epoch \\
\hline Test 1 & 91.0 & 92750 & 92.7 & 7444 & 90.8 & 9200 \\
\hline Test 2 & 90.3 & 174600 & 91.2 & 22261 & 92.4 & 18140 \\
\hline Test 3 & 90.5 & 95000 & 91.4 & 26304 & 90.8 & 15000 \\
\hline Test 4 & 89.6 & 84000 & 91.5 & 8000 & 91.2 & 5400 \\
\hline Test 5 & 89.9 & 17400 & 90.8 & 10657 & 92.5 & 11935 \\
\hline Test 6 & 89.1 & 155600 & 89.9 & 6200 & 92 & 13400 \\
\hline Test 7 & 90.7 & 13800 & 92.4 & 46235 & 92.4 & 12120 \\
\hline Test 8 & 89.5 & 176000 & 90.1 & 25600 & 90.3 & 24400 \\
\hline Test 9 & 89 & 55000 & 93.1 & 4000 & 91.9 & 17000 \\
\hline Test 10 & 91.9 & 100100 & 90.5 & 32286 & 91.9 & 16730 \\
\hline Average & 90.2 & 96425 & 91.4 & 18899 & 91.6 & 14333 \\
\hline RSD_1(\%) & 1.0 & 60.8 & 1.2 & 73.5 & 0.8 & 36.5 \\
\hline RSD_2(\%) & 53.2 & 7.1 & 51.8 & 54.7 & 22.9 & 18.1 \\
\hline
\end{tabular}

-RSD_1 : Relative Standard Deviation over ten different sets of results by applying ten-fold cross-validation tests.

-RSD_2 : Relative Standard Deviation over ten different sets of results by setting up ten different initial weights for ANNs. 


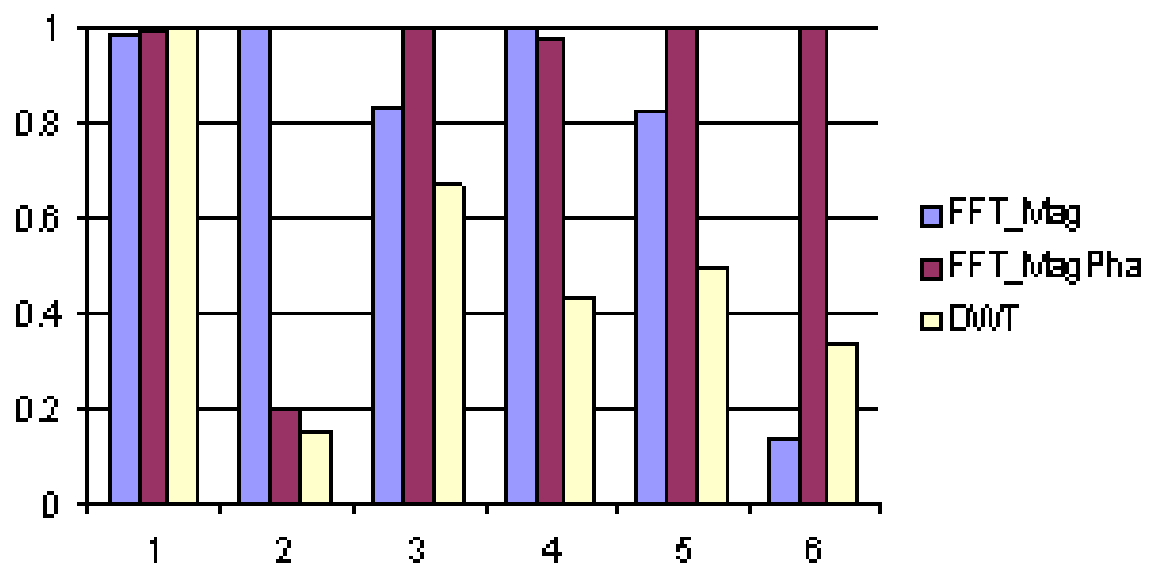

1. The percentage of conect classification overtest data

2. Number of epochs required.

3. Relative standard deviation of the classification results for the lotest sets used inthe ooss validation test.

4. Reltive standard deviation of the classification results for the 10 sets of test by setting $\Psi$ different initial weights on AlNSs.

5. Relative standard deviation of the required epochs forthe 10 test sets used inthe coss validation test.

6. Reltire standard deviation of the requined epochs for the 10 sets of test by setting $\Psi$ different initial weights on AHWs.

Figure 3.2: Relative comparison of performance of ANNs using three different feature extraction schemes: FFT_Mag vs FFT_Magpha vs DWT. 
In order to judge the statistical significance of the test results, we conducted ANOVA [19, 20] tests for the results at the $\mathrm{p}=0.05$ level. The ANOVA test results are summarized in Table 3.2 and their full results are in Appendix A.1. The statistical significance of the results is determined by comparing the $\mathrm{F}$ value produced through $\mathrm{F}$-test with its corresponding F-test critical value. That is, if $\mathrm{F}$ value is bigger than the critical value, the evidence of statistical significance is produced and then the test results can be supported.

Table 3.2: A summarized result of the ANOVA test conducted for the results presented on Table 3.1.

\begin{tabular}{|c|c|c|c|}
\hline \multicolumn{2}{|c|}{ Groups } & \multicolumn{2}{c|}{ F-test } \\
\hline Feature Extraction Method & Result & F value & $\begin{array}{c}\text { F-test } \\
\text { Critical Value }\end{array}$ \\
\hline \multirow{2}{*}{ FFT_Mag, FFT_MagPha, DWT } & epoch & 17.47 & \multirow{2}{*}{3.354} \\
\cline { 2 - 3 } & accuracy & 6.94 & \\
\hline
\end{tabular}

The results from our experiments with three different feature sets suggest the following pairwise comparison. First, does phase information count for something on long signals? That is, using only the magnitude of the FFT sequences as a feature set rather than using the magnitude and phase together as FFT based feature sets (but reduced sampling rate). Secondly, does DWT buy more information than phase information? That is, a comparison between feature extraction schemes using FFT and DWT.

Because we separated the magnitudes component and phase component of complex FFT sequences and rearranged them by concatenation, we can compare this feature extraction technique (FFT_Magpha) with the more generally used FFT based feature extraction technique (FFT_Mag). Our experiments show that the FFT_Magpha provided the better result in classification performance. This experimental result is also supported by its ANOVA test result in Table 3.2. Moreover, clearly a smaller number of epochs is required for convergence (for equivalent error tolerance) in the validation set when using the FFT_Magpha scheme. This result implies that FFT_Magpha is a more efficient FFT based feature extraction approach than FFT_Mag. Therefore, in order to investigate whether DWT can outperform FFT as a feature extraction scheme in this application, it is more appropriate to compare the feature extraction schemes using DWT with the FFT_Magpha approach than with the 
FFT_Mag. We can also say that FFT_Magpha provides the benchmark for the FFT-related feature extraction scheme.

The DWT feature extraction scheme shows a level of classification accuracy similar to that of FFT_Magpha for validation data. However, in terms of the speed of training neural networks, DWT is faster than FFT_Magpha. This result implies that DWT represents a considerable saving in training time. In addition, the significantly small standard deviation between test results and between different runs across the 10-fold cross validation test using DWT, demonstrates that the DWT provides more stability as a feature extraction method than the FFT does.

\subsection{Remarks}

The aim of the investigation presented in this chapter is to establish if DWT-based feature extraction for the ultrasonic shaft signal classification could be more beneficial than FFTbased feature extraction. Other reported previous studies [40, 74] have compared the DWT based features with the FFT with limited feature components. Typically, those previous reports considered only short signals and they paid little attention to the phase components of FFT sequences. In this chapter, an approach to represent both the magnitude and phase of the FFT sequences in a feature vector is suggested and these newly proposed FFT based feature sets were compared not only with the conventional FFT feature sets but also with DWT based feature sets.

More precisely, three feature extraction schemes are extensively tested in this application. Namely, FFT magnitude (FFT_Mag), FFT magnitude and phase combination (FFT_Magpha) and DWT were used to represent the signal data feature. All parameters of the ANN classifiers are kept constant to focus on the feature extractions scheme. Thus, the data features have the same number of components and are presented to ANNs with the same architecture and parameters. The effectiveness of all feature extraction schemes were compared by their classification accuracy for ultrasonic shaft signal echoes. From the analysis of the results the following conclusions can be drawn.

- If the "phase" components of FFT sequences, which can usually be ignored in the 
process of extracting the frequency components, are represented in the FFT based feature vectors, the precision as well as their training and execution time are highly enhanced.

- With respect to classification accuracy, the DWT-based feature extraction method also provides results as good as the FFT_Magpha method.

- the DWT also showed more reliability by producing comparatively stable results in different runs of cross validation tests. This implies that the DWT has potential as feature extraction scheme for training ANNs with arbitrary training data and using the networks for in-field ultrasonic shaft signal classification.

DWT coefficients have some well-known problems, which include that they are not invariant to shifts in the signals and that DWT coefficients yield the relatively large size of the transformed sequences [61, 73]. Even though the experiment in this chapter used DWT without addressing these well-known problems, our experimental results suggest that there are likely benefits to be gained by using DWT as a feature extraction scheme in ultrasonic shaft signal classification. Moreover, we can only expect better results if these problems are addressed. Thus, we expect to find further improvements in classification accuracy with adapted DWT coefficients or more improved feature extraction schemes based on the DWT. 


\section{Chapter 4}

\section{FFT vs DWT using SVM Classifiers}

\subsection{Introduction}

To identify more effective and reliable sets of features for AUSC, we expand the exploration of the FFT and the DWT schemes. These are two top feature extraction schemes. In order to extract feature sets, large numbers of applications employ these two different preprocessing techniques: namely, the use of coefficients from the FFT output or the use of coefficients from the DWT output. Researchers have compared classification performance, as we have discussed before. Especially, for analyzing feature extraction in more complex ultrasonic signals from shafts, we investigated whether the DWT outperforms the FFT in Chapter 3, and this investigation established experimentally that DWT had stronger potential as a feature extraction scheme for feeding ANNs. The experimental results reported in Chapter 3 also show that training data could be selected with higher flexibility and then used in these ANNs for in-field ultrasonic shaft signal classification.

However, this raises the issue about the synergy created by the DWT as a feature selector and the ANN as the classifier; namely, is this a feature extraction that is too much fit for ANN and not useful for other classifiers? That is, can the superiority of DWT be validated 
by the comparison of its classification performance with FFT's only through ANN classifiers? ANNs have been popularly employed as classifiers for AUSC due to their ability of making non-linear decision boundaries. However, considering the many difficulties inherent in the ANN learning paradigm (such as generalization control, overfitting and parameter tuning) we shall be careful in claiming DWT's predominance.

In order to confirm the potential of DWT as an efficient feature extraction scheme for ultrasonic shaft test signals, we made a new comparative experiment involving the Support Vector Machine (SVM) approach instead of ANN models. SVM has gained a strong reputation for its generalization control capability, thus avoiding overfitting, and more confidence can be placed in comparison results using SVM modelling than in those using ANN modelling, especially when there are only a limited number of training examples.

The aim of this chapter is to report results of our investigation into whether DWT can outperform FFT at extracting features in ultrasonic signals from shafts through SVM classification. Because of the properties of SVM (like risk minimization), we see this as stronger validation of the superiority of DWT. The classification results from both schemes (FFT and DWT) are also analyzed to investigate whether different feature extraction schemes affect the classification performance in different classes. The analysis and investigation deliver results that indicate which schemes work best for a class. This information sets the baseline for later work (subsequent chapters). For example, before our experiments it was unknown whether one scheme of feature extraction would produce more accurate classification for a certain type of echo than another scheme. It also was unclear if the roles reverse for other types of echoes. As a consequence of this, we will be in a position to build more advanced hybrid classifiers using both feature schemes in later chapters.

We organize the rest of this chapter as follows. Section 4.2 briefly describes SVM as a learning algorithm. Section 4.3 explains the overall procedure for our experiment and details the steps involved in the experiment. It describes the procedure of preprocessing and constructing a database for our experiment, and the application of the SVM learning model. Section 4.4 analyzes the experimental result of both schemes and compares and discusses their performance, followed by conclusions from this experiment in Section 4.5. 


\subsection{SVM Classifier}

The SVM is a machine learning tool for performing various tasks related to supervised classification or regression. In particular, SVM classifiers learn a particular classification function from a set of labelled training examples. The training set consists of $\mathrm{n}$ training examples, with each example described by a $d$-dimensional vector, $x \in \mathbf{R}^{d}$, labelled as belonging to one of two categories, $y \in\{-1,1\}$ referred to as the "positive class" and the "negative class". Following training, the result is an SVM that is able to classify a previously unseen and unlabelled instance, $x$, into a category based on examples learnt from the training set $[7,22]$.

Geometrically, the SVM classifier aims to construct a hyperplane that divides $\mathbf{R}^{d}$ in two, with all training examples in the positive class on one side of the hyperplane, and all examples in the negative class on the other side. Although there may exist an infinite number of hyperplanes that correctly separate the training data, the best SVM classifier is obtained by finding the hyperplane with the maximum "margin". The margin is defined as the distance between the closest training examples in the positive and negative classes to the separating hyperplane. The training examples that determine the margin are known as "support vectors". A trained Support Vector Machine classifies unlabelled points according to the side of the hyperplane on which they fall [13].

In a typical training set, there will be some examples that unavoidably fall in the wrong side of the hyperplane's decision boundary. In this situation, the separating hyperplane is found by simultaneously maximizing the margin between the two classes while minimizing the penalty associated with the misclassifications in the training set. The optimum hyperplane, defined by $(w \cdot x)+b=0$, is found by solving the following quadratic programming problem:

$$
\begin{array}{r}
\min _{\omega, b, \xi} \frac{1}{2}\|\omega\|+C \sum_{i=1}^{n} \xi_{i} \\
\text { subject to } \quad y_{i}\left(x_{i} \cdot \omega+b\right) \geq 1-\xi_{i} \\
\xi_{i} \geq 0 \quad i=1, \ldots, n
\end{array}
$$

where $\xi, i=1, \ldots, n$ are slack variables introduced to allow for examples that fall on the 
wrong side of the hyperplane, and $C$ is a positive parameter that controls the trade-off between maximizing the margin and minimizing the training error.

While the original optimal hyperplane algorithm [99] was a linear classifier, nonlinear problems can be handled by using a kernel function to map the data into a different space where a hyperplane can be used to do the separation [7]. In other words, the inner-product kernel $K\left(x_{i}, x_{j}\right)$ is used to construct the optimal hyperplane in the feature space without considering the feature space itself in explicit form. The concept of a kernel mapping function allows SVM models to perform separations even with very complex boundaries. Many kernel mapping functions can be used and four basic kernels which have been commonly used are as follows:

- linear: $K\left(x_{i}, x_{j}\right)=x_{i}^{T} x_{j}$

- polynomial: $K\left(x_{i}, x_{j}\right)=\left(\gamma x_{i}^{T} x_{j}+r\right)^{d}, \gamma>0$

- radial basis function $(\mathrm{RBF}): K\left(x_{i}, x_{j}\right)=\exp \left(-\gamma\left\|x_{i}-x_{j}\right\|^{2}\right), \gamma>0$

- sigmoid: $K\left(x_{i}, x_{j}\right)=\tanh \left(\gamma x_{i}^{T} x_{j}+r\right)$

where $\gamma, r$ and $d$ are kernel parameters.

Based on the theory of SVM as explained above, SVM provides efficient and powerful classification algorithms which are capable of dealing with high dimensional input features, and with theoretical bounds on the generalization error and sparseness of the solution provided by statistical learning theory. Classifiers based on SVM have few parameters requiring tuning, are simple to implement, and are trained through optimization of a quadratic cost function. Furthermore, SVM based solutions are sparse in the training data, and are defined only by the most informative training points. 


\subsection{Experiment}

\subsubsection{Overall Process}

This chapter has a similar structure to Chapter 3. It too compares the FFT and the DWT as feature extraction schemes. The overall experimental setting for this chapter also consists of similar processes to the processes in Chapter 3. However, some detailed processes are different from the experiment in the previous chapter and especially, this chapter concerns the use of SVM as a learning algorithm. The flowchart of the experiment for this chapter is in Figure 4.1.

The first process starts from selecting signal segments of interest from the whole ultrasonic A-scan signals. In order to apply a consistent way of signal segmentation which is necessary for suppressing time-variance problems of DWT, we used a systematical echo-capturing method with zero-padding. The time-variance problem of DWT and the method we used to solve that problem are discussed with more details in Chapter 5. In order to build training data sets, we also recorded whether the signal originated from a crack or not and whether it was a primary or secondary (mode-converted) echo. Once an initial database had been built, the data was mapped to feature domains. Two different feature extraction techniques $\left(\mathrm{FFT}^{1}\right.$ and $\left.\mathrm{DWT}\right)$ were applied to extract more informative features from the selected signal features. Because we apply two different feature extraction techniques, we generate two different sets of feature training data. We use these two sets of feature training data for training two separate SVM models. Both SVM models use Radial Basis Function $(\mathrm{RBF})$ as their kernels and their classification performances are validated through tenfold cross-validation. We describe more details of the experimental steps in the following subsections.

\footnotetext{
${ }^{1}$ The FFT here is the approach that includes phase information as we established earlier in Section 2.3.2. This results in the best alternative in the FFT suite.
} 


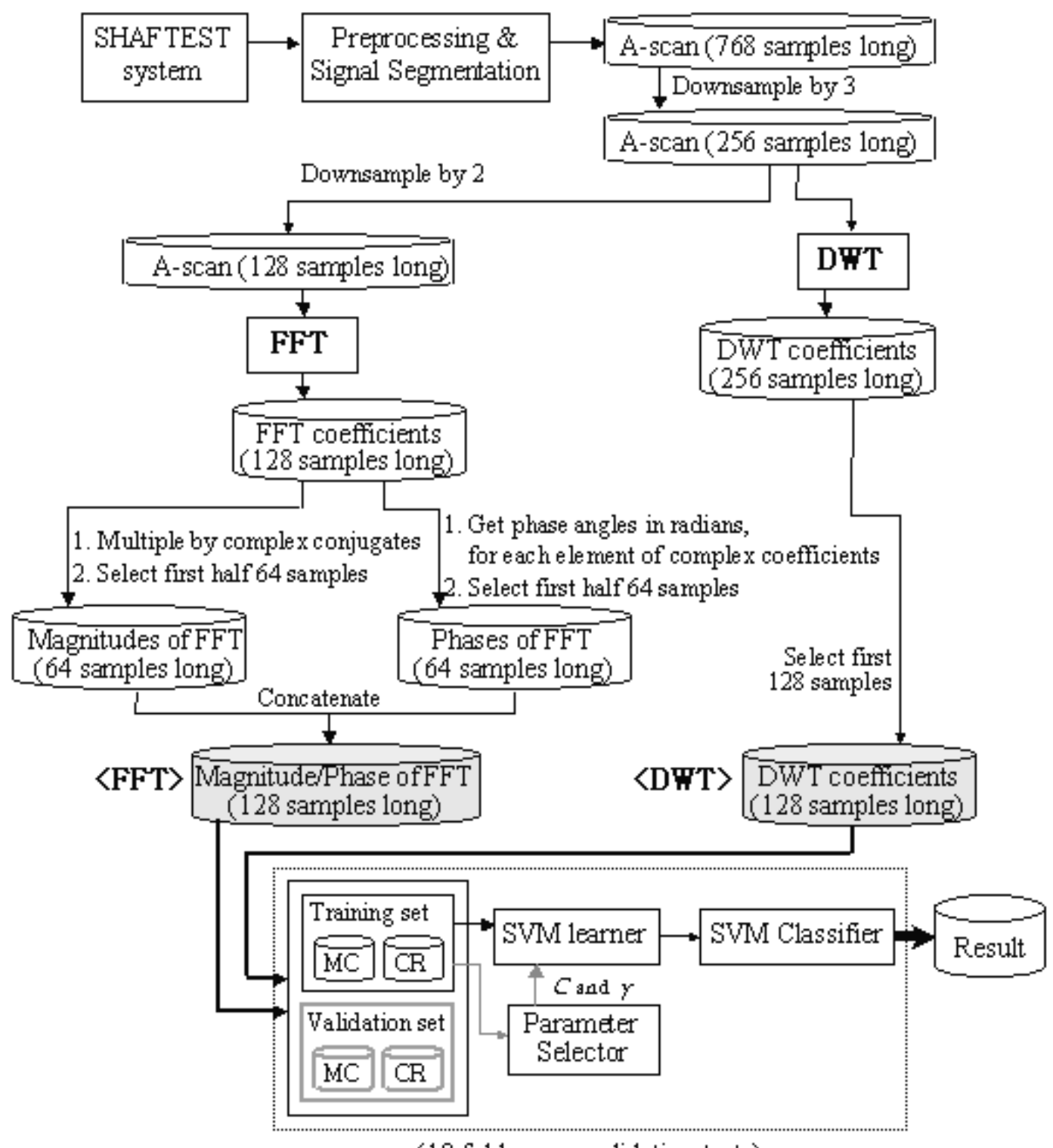

(10 folds cross validation tests)

Figure 4.1: An overall procedure for the experiment for SVM classification. 


\subsubsection{Preprocessing and Feature Extraction}

We collected A-Scan data using a probe set to $2 \mathrm{MHz}$ frequency from eight different shafts ranging between $100 \mathrm{~mm}$ and $1300 \mathrm{~mm}$ long. Five of these shafts contained cracks and the rest were clean shafts. We were able to capture mode-converted echoes from all of these shafts except for one $100 \mathrm{~mm}$ calibration block.

Even though the overall procedure for collecting A-Scan data is nearly the same as described in Chapter 3, we used a $2 \mathrm{MHz}$ ultrasonic probe to fire and receive the pulse this time, while a $5 \mathrm{MHz}$ probe had been used when collecting data for ANN experiments. This is due to the fact that these two groups of experiments were conducted at different times and the tool for capturing A-Scan signals (ShafTest ${ }^{T M}$ ) was updated into the new version during the gap period. The new system does not support the use of $5 \mathrm{MHz}$ probe any more, so we could not avoid inconsistency between the two experiments.

In addition, more effort was made to construct a varying range of ultrasonic shaft signals than before. Firstly, we changed the grouping of the shafts from which A-Scan data are to be collected. Three old plain shape shafts were replaced with three new shafts which had been used in a real field and had more complicated features (e.g. spines, holes or abnormal cracks). When we collected A-Scans for constructing this new A-scan data pool, we also made efforts to remove some redundancy of training data that existed in previous data. Though we can not say the redundancy which the previous data set contained was due to collecting exactly same A-Scans, a number of A-scans were captured in a very close position thus had no big difference in terms of their signal features. By removing such redundancies or duplicates, we built more varying range of data thus expected that the newly constructed A-scan database could reflect better the variance of shaft signal features.

For the selection of the signal's region of interest that could effectively solve time-variance problems in DWT, we developed a methodology for gating a signal section to single out an echo (detailed in Chapter 5). In this way, we determined a border of echo-only area for every signal echo using a consistent systematic rule, and the effects of the background noise was minimized by excluding the neighboring background grass area through zero-padding. Through this process, we first produced a set of time-domain signal vectors with length 768 sample points and we down-sampled them into 256 samples to reduce the dimension of the 
input vector (pattern) for the SVM classifier.

We recorded the class for every pattern vector, whether the echo was caused by Crack $(\mathrm{CR})$, Mode-Conversion (MC) or Backwall-Reflection (BR). As mentioned in Chapter 3, type BR is considered to be more easily distinguished than CR or MC [23] and moreover, the main concern in the field of shaft inspection is that the signal echoes caused by CR can be confused by fainted echoes caused by MC and vice versa. Therefore, in our experiments, different feature extraction schemes are compared by the classification performance in classifying the received signal echoes just as those from CR or MC. For this purpose, we chose randomly $50 \mathrm{CR}$ signals and $69 \mathrm{MC}$ signals from the sample pool to use in the experiment. Thus, a data set with a total of 119 time-domain signals became the basis for further processing.

The next step was converting the time-domain data into different domain feature sets which would later become the input to the SVM classifiers. For our purpose, we converted time-domain signal data into the frequency domain using the FFT and also into the time-frequency domain using the DWT. As mentioned in Chapter 3, there are some difficulties in evaluating claims in earlier research from similar scenarios on the superiority of DWT coefficients over FFT coefficients as features for the ultrasonic signal classification. The reason is that most previous comparison studies used only the magnitude component of the FFT-transformed signals, and their phase components were excluded through the process of using FFT coefficients as feature vectors for a classifier. To make sure we did not fall into this trap as well, we developed an FFT-based feature extraction scheme, namely "FFT_Magpha", that includes phase information. We showed this was superior while using exactly the same dimensionality reduction as in Chapter 3. This means we have a state-ofthe-art FFT method for our application. We found this variant of FFT most effective in that it attaches phase information to each selected magnitude component. Because of the success of this design of FFT feature vector, as the most effective FFT variants, it is the one we use for comparison between FFT and DWT as feature extraction schemes for SVM classification of ultrasonic shaft signals.

For the comparison study in this chapter, the gate-selection preprocessing delivers vectors whose components are 256 values of long time-domain data. We down-sampled them into 128 values and then transformed them by the FFT. The sequence FFT of a signal is a sequence of complex numbers which consists of magnitude and phase of the transformed 
sequence. As the last half of the sequence of FFT corresponds to complex conjugates of the components in the first half of the sequence, the FFT of 128 values (points of a discrete signal) yields 64 magnitude components and 64 phase components. The concatenated sequences of magnitude components and phase components becomes the 128 dimensional pattern vector for SVM classification.

On the other hand, we also converted the 256 values that constitute the time-domain signal patterns by the DWT. The procedure of applying the DWT scheme for the experiment in this chapter coincides with the procedure in Chapter 3. That is, it starts from decomposing discrete time signals into their coarse and detail information by passing them through a high-pass and a low-pass filter respectively. This decomposition is repeated several times only for low-pass filtered signals. We concatenate the outputs from high-pass filters in each decomposition level. Since these will be the DWT coefficients, we start with the coarsest coefficients. This is reasonable since these last coefficients of the DWT indicate little information and represent mainly noise. These vectors with length 128 values of DWT coefficients are stored as the DWT features. For our experiment, we also applied Daubechies wavelets [26] for filtering.

\subsubsection{SVM Classification}

As mentioned before, this chapter aims at investigating whether the DWT is superior to the FFT as a feature extraction scheme for the ultrasonic shaft test signals using the SVM classification approach. In Chapter 3, we reported that the DWT outperformed the FFT at extracting features in ultrasonic signals from shafts by comparing their classification performance using ANN classifier models. However, it is important to have enough training patterns for effective ANN classifier learning. For a more reasonable comparison between FFT and DWT, therefore, it is necessary to employ a more efficient classifier in order to avoid the danger of overfitting inherent in high dimensional feature spaces. Because of their risk minimization and the solution in dual space, SVM classification is a very good choice to determine, as far as feature extraction methods are concerned, whether DWT is generally superior.

SVM has gained wide acceptance as a powerful supervised learning algorithm based on sta- 
tistical learning theory and it has shown remarkably robust performance in several pattern recognition applications $[38,42,72]$. The most important property SVM offers is the generalization control capability, the avoidance of overfitting, which is a very important issue in defect detection systems like an AUSC system for shaft test [24].

Among various kernels proposed, for our experiment, we employed RBF kernels for constructing SVM classifiers. The RBF kernel non-linearly maps samples into a higher dimensional space, so it, unlike the linear kernel, can handle the case when the relation between class labels and attributes is nonlinear. Furthermore, previous researchers suggest that the linear kernel can be considered as a special case of RBF [45] and the sigmoid kernel behaves like RBF for certain parameters [56]. Another reason for selecting RBF kernel is the number of hyperparameters which influences the complexity of model selection. The polynomial kernel has more hyperparameters than the RBF kernel. In addition, the RBF kernel has fewer numerical difficulties than other kernels. The value of $K_{i j}$ is retained in a range of $0<K_{i j} \leq 1$ in the RBF kernel function. However, in polynomial kernels, the values may go to infinity $\left(\gamma x_{i}^{T} x_{j}+r>1\right)$ or zero $\left(\gamma x_{i}^{T} x_{j}+r<1\right)$ while the degree is large. Moreover we must note that the sigmoid kernel is not valid (i.e. not the inner product of two vectors) under some parameters [100].

The next procedure after the selection of kernel function is selecting parameters required for the kernel function. Two parameters: a penalty parameter $C$ and kernel parameter $\gamma$ are required for RBF kernels. It is not known beforehand which $C$ and $\gamma$ are the best for one problem. Consequently we need to do some kind of model selection using parameter searching. Among several previously suggested methodologies for searching the best parameters for classification problems, we used a grid searching algorithm [37] where pairs of $C$ and $\gamma$ are tried and the one with the best 10-fold-cross-validation accuracy is picked. For this parameter searching method, we used a grid searching tool grid.py which is provided through LIBSVM interface [15] that is a software for support vector classification. By using the grid searching tool, we tried pairs $(C, \gamma)$, where $C=2^{-5}, 2^{-4}, \cdots, 2^{15}$ and $\gamma=2^{-15}, 2^{-14}, \cdots, 2^{5}$, over each set of feature data (FFT patterns and DWT patterns). The procedure for constructing these two different feature sets is described in Section 4.3.2.

Following the parameter searching method, we set $C$ to the value 2.0 and $\gamma$ to the value 0.125 for the DWT-based patterns while the value 32.0 was set for $C$ and 0.0078125 for 
$\gamma$ in the case of FFT-based patterns. The results of the grid searching are graphically presented in Appendix B. Different SVM parameters are used in building each model using FFT or DWT as a feature extraction method. We tried to find the more data-dependent parameters which showed the best performance for each different pattern (FFT patterns and DWT patterns), rather than selecting one common parameter set. We believe that the comparison of two classifiers built using those locally-best-parameter sets is fairer than the comparison of two classifiers constructed using one common parameter set.

The input samples were shuffled and randomly divided up into 10 sets. In turn, 9 of these were used to train the SVM classifier, and the remaining set was used to validate the constructed SVM classifier. We used OSU-SVM [59], which is a SVM toolbox for the MATLAB numerical environment, to construct SVM classifiers and test them. This 10 fold cross-validation test was carried out on two SVM models, which are trained by two feature sets: FFT and DWT. The performance of the two different schemes can be compared by the result of each cross-validation test.

\subsection{Results and Analysis}

As mentioned in Section 4.3.2, the data sets used in the experiment using ANNs (Chapter 3) and in the experiment using SVMs (presented in this chapter) are in fact different. The data set used for the ANN experiment is much more limited while the data set used for the SVM experiment is more realistic, larger and richer in its classes and scenarios. Moreover, the ANN experimental results (accuracy) were gained through a rather manual procedure in order to observe comprehensively the moment of overfitting for each ANN test. ${ }^{2}$ The manual procedure for gaining the accuracy for each ANN test did not include the way of gaining results for each class thus the results of ANN experiments (presented in Table 3.1) do not include the class results. ${ }^{3}$

On the other hand, to compare and analyze the performance of each feature extraction

\footnotetext{
${ }^{2}$ The treatment of the over-fitting problem and the procedure performed to finding the optimal architecture of ANN models is described in Section 3.3.3.

${ }^{3}$ We designed the procedure in that way at the time when we conducted the ANN experiments, because the main purpose of the experiment of Chapter 3 was gaining and comparing the overall performance of ANNs using FFT and DWT and the difference between class results were not considered importantly then.
} 
scheme for ultrasonic shaft signal classification using SVMs, we gained not only the overall classification results but also the class results. That is, we recorded the classification accuracy for each of 10 test runs as well as the average accuracy, and also analyzed how many instances of each class (CR and MC) were classified correctly. We summarize the classification results (as accuracy percentages) in Table 4.1. Figure 4.2 offers a visual comparison between the classification performance of the two different feature extraction schemes. It displays the corresponding histograms of relative values for the results shown in Table 4.1. In order to judge the statistical significance of the test results, we also conducted ANOVA testsfor the results at the $\mathrm{p}=0.05$ level. The test results are summarized in Table 4.2 and the full results are in Appendix A.2.

Table 4.1: Accuracy in classifying each class (CR and MC) for two different feature extraction schemes: FFT vs DWT.

\begin{tabular}{|c|c|c|c|c|c|c|}
\hline & \multicolumn{3}{|c|}{ FFT } & \multicolumn{3}{|c|}{ DWT } \\
\hline & CR & $\mathbf{M C}$ & Overall & CR & $\mathbf{M C}$ & Overall \\
\hline Test 1 & 57.7 & 100 & 79.4 & 92.3 & 78.4 & 84.1 \\
\hline Test 2 & 83.3 & 100 & 86.7 & 90.2 & 75 & 88 \\
\hline Test 3 & 60.7 & 94.3 & 79.4 & 92.9 & 85.7 & 84.1 \\
\hline Test 4 & 57.9 & 90 & 61.9 & 100 & 82 & 87.5 \\
\hline Test 5 & 72.5 & 91 & 80 & 81 & 90 & 84.4 \\
\hline Test 6 & 62.5 & 100 & 82.5 & 91.7 & 84.6 & 87.3 \\
\hline Test 7 & 73.1 & 91.9 & 84.0 & 100 & 86.5 & 92.1 \\
\hline Test 8 & 63.6 & 93.5 & 81 & 100 & 75 & 81.3 \\
\hline Test 9 & 76.2 & 72.8 & 75 & 90 & 100 & 93.8 \\
\hline Test 10 & 64.3 & 91.7 & 79.7 & 92.9 & 77.8 & 84.4 \\
\hline Average & 67.2 & 92.5 & 79 & 93.1 & 83.5 & 86.7 \\
\hline
\end{tabular}

Even though we do not aim at a comparison between the ANNs' performance (with the early version of data set) and SVMs' performance (with the improved version of data set) in this thesis, we also conducted an SVM experiment using the old data set which had been used for the ANN experiment in Chapter 3 and the results are shown in the shaded sections 


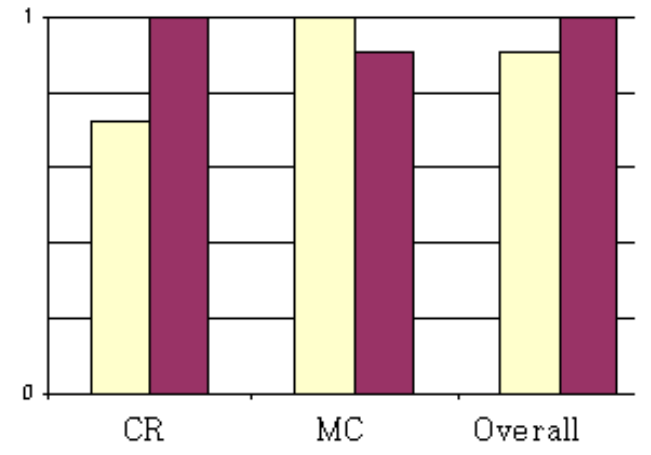

FFT

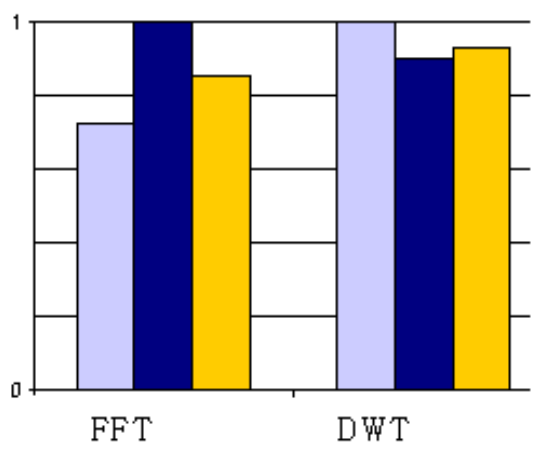

$\square \mathrm{CR}$

MC $\square$ Overall

Figure 4.2: Relative comparison of performance using two different feature extraction schmes: FFT vs DWT.

Table 4.2: A summarized result of the ANOVA test conducted for the results presented on Table 4.1 .

\begin{tabular}{|c|c|c|c|}
\hline \multicolumn{2}{|l|}{ Groups } & \multicolumn{2}{|r|}{ F-test } \\
\hline $\begin{array}{c}\text { Feature Extraction } \\
\text { Method }\end{array}$ & Class & F value & $\begin{array}{c}\text { F-test } \\
\text { Critical Value }\end{array}$ \\
\hline FFT & \multirow{2}{*}{$\mathrm{CR}, \mathrm{MC}$} & 46.74 & \multirow{5}{*}{4.414} \\
\hline DWT & & 9.82 & \\
\hline \multirow{3}{*}{ FFT, DWT } & CR & 61.96 & \\
\hline & $\mathrm{MC}$ & 6.62 & \\
\hline & Overall & 9.8 & \\
\hline
\end{tabular}


of Table 4.3. This table also includes the corresponding ANN test results extracted from Table 3.1. These results confirm differences in the feature extraction methods, while the classifier is now SVM.

Table 4.3: The classification performance of ANNs and SVMs using three different feature extraction schemes with the earlier version of the test data set

\begin{tabular}{|c|c|c|c|c|c|c|}
\cline { 2 - 7 } & \multicolumn{2}{c|}{ FFT_Mag } & \multicolumn{2}{c|}{ FFT_MagPha } & \multicolumn{2}{c|}{ DWT } \\
\cline { 2 - 7 } & ANN & SVM & ANN & SVM & ANN & SVM \\
\hline Test 1 & 91.0 & 91.4 & 92.7 & 95.2 & 90.8 & 87.8 \\
\hline Test 2 & 90.3 & 91.7 & 91.2 & 87.7 & 92.4 & 87.6 \\
\hline Test 3 & 90.5 & 90.2 & 91.4 & 94.0 & 90.8 & 94.4 \\
\hline Test 4 & 89.6 & 88.3 & 91.5 & 92.9 & 91.2 & 96.4 \\
\hline Test 5 & 89.9 & 88.2 & 90.8 & 87.4 & 92.5 & 95.5 \\
\hline Test 6 & 89.1 & 81.5 & 89.9 & 97.3 & 92 & 90.4 \\
\hline Test 7 & 90.7 & 92.3 & 92.4 & 90.4 & 92.4 & 98.7 \\
\hline Test 8 & 89.5 & 88.2 & 90.1 & 92.3 & 90.3 & 96.4 \\
\hline Test 9 & 89 & 86.1 & 93.1 & 91.4 & 91.9 & 97.9 \\
\hline Test 10 & 91.9 & 92.3 & 90.5 & 91.7 & 91.9 & 96.7 \\
\hline Average & 90.2 & 89.0 & 91.4 & 92.0 & 91.6 & 94.2 \\
\hline
\end{tabular}

Through the analysis of the experimental results, we gain several noteworthy points as follows:

- As shown in Table 4.1, the SVM classification performance of DWT is superior to that of FFT in every different test run. This matches the result of the previous comparison applying ANN models. Even though the experimental settings are different from those for Table 4.1, the SVM results shown in Table 4.3 also present similar phenomenon in terms of the differences in the feature extraction methods. ${ }^{4}$ It implies that DWT is a

\footnotetext{
${ }^{4}$ It is noticed that the SVMs' performance presented in Table 4.1 is comparatively lower than what presented in Table 4.3. We assume that it is caused by the difference in the test data set used for two experiments. A number of diverse types of A-Scans (provided from shafts that had been used in the real mining field) were added for the test data set used in the later experiment (Table 4.1).
} 
more reliable feature extraction scheme; that is, it can be employed for constructing a better classifier for in-field ultrasonic shaft signal tests.

- As shown in Table 4.1 and Figure 4.2, we can observe some differences for specific classes of echoes when reflecting upon the classification result from using each feature extraction scheme. DWT shows better performance in classifying CR than MO, while FFT shows a preference for the other way around. That is, using DWT one rarely gets a CR incorrectly classified (either a CR as something else or something else as a $\mathrm{CR}$ ). Symmetrically, using FFT one rarely gets an MO incorrect.

- This result can provide useful prior knowledge when constructing a hybrid AUSC system for testing shafts using a combined feature extraction scheme. In this system, the FFT, in spite of lower accuracy for overall classification, could complement the decisions based on DWT features. Consequently, this result becomes a motivation of the hybrid classifier approach presented in Chapter 6 .

\subsection{Remarks}

Experimental evidence presented in Chapter 3 concluded that DWT provides faster and more reliable feature extraction for ANN classifiers for long shaft test signals. In the current chapter, we demonstrate that DWT is not only highlighting more adequate vectors for ANN, it is more general and in particular it also improves the performance of Support Vector Machines (SVM). The results reported here dissipate any doubt that the DWT feature extraction methodology is too far suited for ANN. In fact, we have shown that the DWT features are indeed superior because they provide better classification with SVM, for which statistical properties indicate robustness in its construction. Also, we have observed a slight facility when specific classes of echoes are concerned with different combinations of feature extraction and classifier. This leads into a hybrid classifier that will improve overall performance by giving more weight to the more trustworthy sub-classifier. 


\section{Chapter 5}

\section{Echo Gating Techniques for Ultrasonic A-Scans}

\subsection{Introduction}

The literature suggests that the DWT coefficients of ultrasonic test signals are useful features for input into classifiers due to their effective time-frequency representation of nonstationary signals. We demonstrated this potential for using DWT in the case of ultrasonic shaft test data in Chapter 3 and Chapter 4. However, there is little enthusiasm for using DWT coefficients as a feature set because wavelet coefficients are variant to shifts in measured time-domain signals. To overcome this time-variance problem, the most general method in use today is to calculate the total energy values of all signal sequences in each coefficient level [73, 95]. This method, however, ignores the shape of the energy distribution in each level of DWT coefficients and thus uses only sums of energies in each level as features for classification. This implies potentially very large information loss.

Therefore, as another approach to overcome the time-invariance problem of DWT coefficients, in this chapter, a new technique to derive a preprocessing method for time-domain signals is presented. The main idea of this new technique is retaining the shape of energy distributions while simultaneously retaining a consistent horizontal (time-domain) position 
on the targeted signal echo. In order to implement this approach, two alternatives are suggested. The first employs a systematic rule in capturing (gating) a signal echo to provide an automated and consistent feature collection. The second singles out only an echo from the whole A-scan signal. This second method, especially, provides the solution to detecting low energy level echoes which are likely to be hidden by background noise of ultrasonic signals.

For long A-scans on shafts, one must perform gating to capture a segment for analysis. This adds special importance to our method, since it accomplishes the horizontal positioning of Ascans and the systematic signal-capture. The selection of this position should be consistent because this decision usually becomes the first stage of an automatic ultrasonic classification system which all further processing relies upon.

Several methods for consistent selection and horizontal positioning in ultrasonic A-scans have been previously explored, but most methods are fit for the analysis of short signals taken from plate surfaces or pipe surfaces that have no side walls and no mode-converted echoes to contend with $[74,89]$. In many cases, mode-converted echoes emerging from the special geometric properties of shafts are likely to appear multiple times during a short time period. Thus, the multiple secondary echoes may be very close to each other in an A-scan signal. In this case, gating an echo section with regular width is impractical. Once more, the approach suggested in this chapter aims at providing a new method for deciding the horizontal positioning for A-scan signal echoes, which can be applied robustly to echoes with different widths and to echoes in close proximity to each other.

In this chapter, we introduce such a new method for selecting the horizontal region of interest, and report on the investigation into whether this new method outperforms other generally used methods for extracting features in ultrasonic signals from shafts. In order to test the new method, the signal sections selected by various methods are transformed by two different DWT methods and the resulting features are used to train two different types of classifiers: artificial neural networks (ANNs) and support vector machines (SVMs). We compare and analyze the classification performance of each trained classifier.

The rest of this chapter is organized as follows. Section 5.2 details the issues in gating a signal echo which will be used for further processing. Section 5.3 introduces the new method for determining an echo border to single out only one signal echo. Section 5.4 explains the 
procedure for the experiment to compare the effectiveness of the newly developed method with other generally used methods. That is, it describes the procedure of preprocessing and feature extraction for the experiment. Section 5.5 analyzes the experimental results and compares and discusses their performance issues. This is followed by conclusions from these experiments in Section 5.6.

\subsection{Problems}

The very first step of an AUSC system is to capture A-scans. These captured A-scan waveforms are usually displayed in a window. Current practice must classify each echo, and to do so it singles out each echo from A-scans using gates. This builds up the database of such echoes that when classified will characterize the corresponding different types of flaws. Gating is necessary for reducing the size of the data. This is a type of dimension reduction that makes it feasible to classify each echo. Figure 5.1(a) shows an example of time-gating on a digitally captured ultrasonic A-scan. The gate is presented by two vertical lines on the figure. Figure 5.1(b) shows an enlarged look of the signal segment singled out by the gate presented in Figure 5.1(a) and this signal segment will be used as a signal pattern.

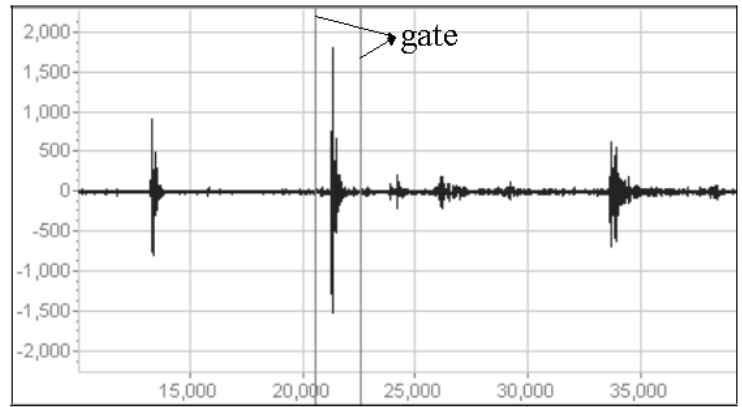

(a)

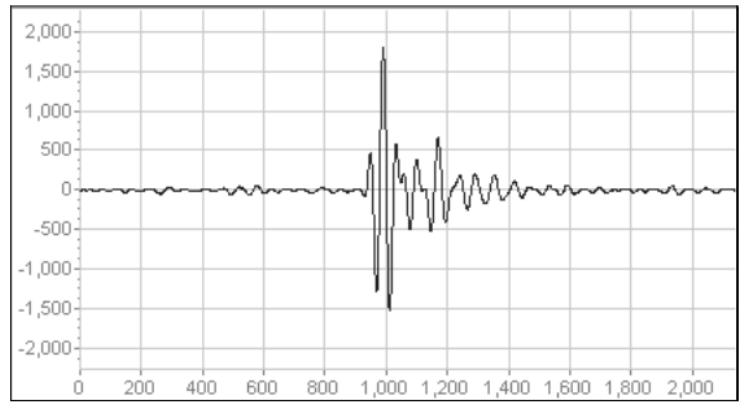

(b)

Figure 5.1: (a) An example of a signal echo gated on the captured ultrasonic A-scan signal. (b) A signal echo singled out.

A general rule for gating a subsection from the originally captured A-scan is to gate segments of A-scans with regular size. The segment must be long enough to have one signal echo in each segment. Thus, it must be long enough that the entire echo can be passed to the classifier with little information loss. It must be short enough to reduce the size of 
the data and the dimension of the classifier. This is the dilemma to be solved here. The gated areas of A-scans can usually be positioned to include echo sections of high amplitude and background grass sections with comparatively low amplitude. The reliability and consistency of positioning and selection of the horizontal position of the gated window are important issues because all further feature extraction process relies on it. A method for horizontal positioning must systematically generate the training set for learning a classifier and replicate the extraction of features (from unseen patterns) that will be fed to the classifier.

Having a consistent method for gating is necessary especially when DWT coefficients are used as features. DWT coefficients can display large variance depending on how flaw signals are gated. An example of this problem is presented in Figure 5.2.

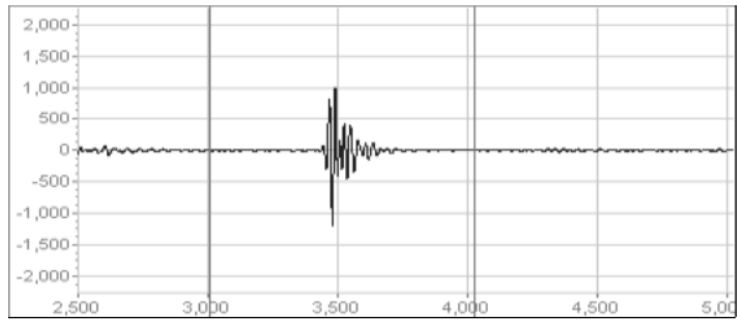

(a)

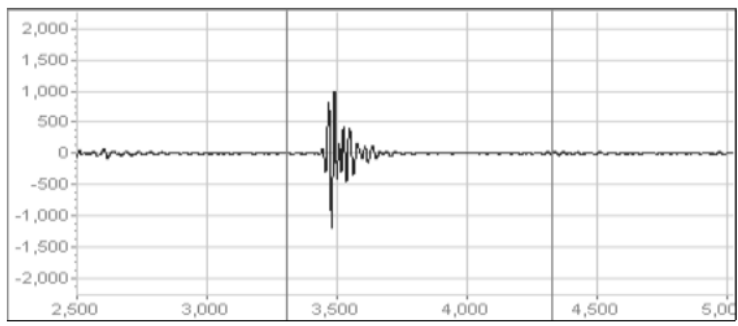

(c)

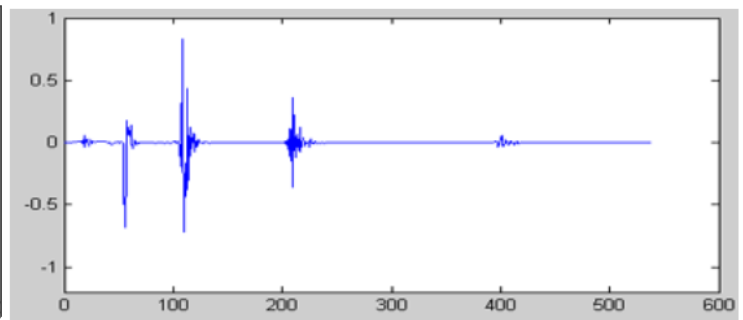

(b)

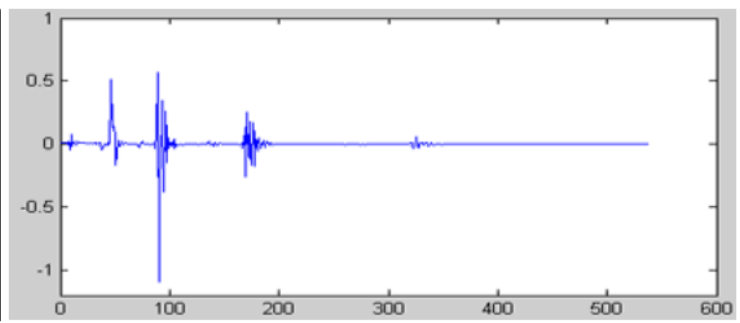

(d)

Figure 5.2: Two different gating methods ((a) and (b)) applied to one flaw signal and their corresponding DWT coefficients ((c) and (d)).

Various efforts have been suggested and applied to overcome the time-invariance problem exhibited by DWT. The best known idea obtains sums of total energy for each coefficient level and passes these as features. This method still has a problem in that the summed energy in each level can vary significantly depending on the horizontal positioning of the gated window [95]. Although this idea of using the summed energy of several members of coefficients in each level has been around for some time, it does not provide a satisfactory 
answer to the problem of which members of coefficient levels are to be selected. The clustered-DWT (CDWT) method was introduced [73] as a solution to this problem. This method applies an unsupervised clustering method to decide which members are to be summed. Even though the clustered-DWT method is reasonable, using irregular signal gating as base for obtaining its source coefficients can be hazardous.

The generally consistent method for A-scan signal gating is to gate a signal section in which the signal peak is positioned in the center. This positioning method works well when the energy distribution of the targeted signal echo has a smooth bell shape. However, this is not a suitable method for dealing with skewed distributional echoes or echoes with more than one peak. Especially, it is problematic in cases where a series of small size echoes are positioned very closely. In this case, regular gating size results in the inclusion of more than one echo. This case is especially frequent when the multiple secondary echoes (mode-converted echoes) are present in shaft test signals. Unfortunately, the challenge is to distinguish this case from the very similar situation where a fainted echo from a real crack exists among several of these closely positioned echoes.

Ideally, one would like to gate exactly the segment that includes the echo energy (excluding exactly the background grass). To realize this goal two main problems are faced:

1. How to decide (in the time dimension) when the echo starts and at which point it ends.

2. If it were possible to decide reliably on starting and ending points, then the width of each gate (segment) would vary for each selected echo. This creates input vectors of different dimension for the classifiers because the selected signal is the basis for construction of the input vectors.

We suggest the application of zero-padding to homogenize the selected echoes to the same width.

To achieve a reliable method for extracting the segment where the echo's energy is manifested, the background noise needs to be treated properly since it is usually distributed irregularly on the entire A-scan. As displayed by Figure 5.3, a flaw echo or mode-converted 
echo can have very small amplitude compared to an initial pulse or back-wall echo. These small amplitude echoes are not easily detected, especially in environments with high background noise. It is generally recommended to normalize a flaw signal, setting the maximum peak to 1 , as a remedy for avoiding the variance in gain settings. However, in environments with high noise, this also increases the background noise. In many cases, this masks the echo among the noise and thus fails to detect the echoes corresponding to flaws.

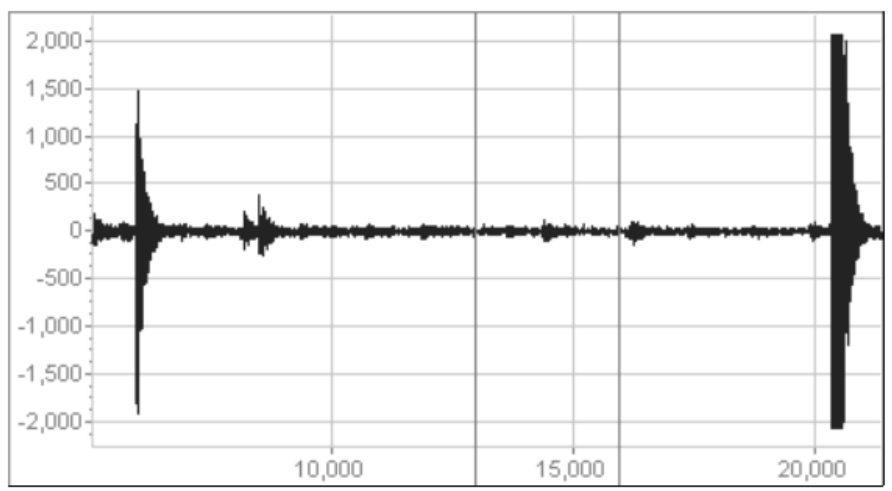

(a)

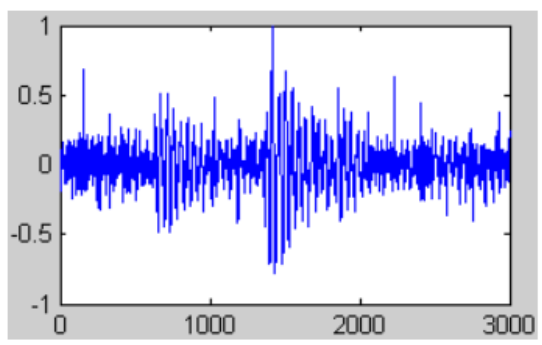

(b)

Figure 5.3: An example of a small amplitude echo and its normalized representation. A flaw echo or a mode converted echo can exhibit very small amplitudes compared to the 'Initial Bang' echo or the back-wall echo; so these small amplitude echoes may not be detectable. (a) An example of a small amplitude echo. (b) A normalized representation of the gated echo.

Therefore, an ideal procedure for extracting an echo-only section from the originally captured A-scan (not including the grass area) in a noisy environment is first to suppress background noise using a noise filtering method, and then to apply a specific decision rule for finding a starting point and ending point for an echo.

\subsection{Echo Border Decision Method}

The technique introduced in this chapter consists of two phases. The first phase is to detect echoes among the input which is a long A-scan captured in digital form. In this chapter, a method to find echoes is described based on the thresholding method which typically has been applied to remove noise. Actually in later phases the noise is removed 
by alternative methods. In particular, the second phase receives as input the original background-noise-added A-scan, with decisions of where echoes have been detected. This second phase produces echo-only segments. These segments are determined by applying a procedure to single out (isolate) echoes. We explain the entire procedure shortly, but we present a graphic flowchart in Figure 5.4. It should be noted that this process can be automated and as a consequence implemented in such a way that it could be embedded into an automatic NDT tool, thus reducing manual operation.

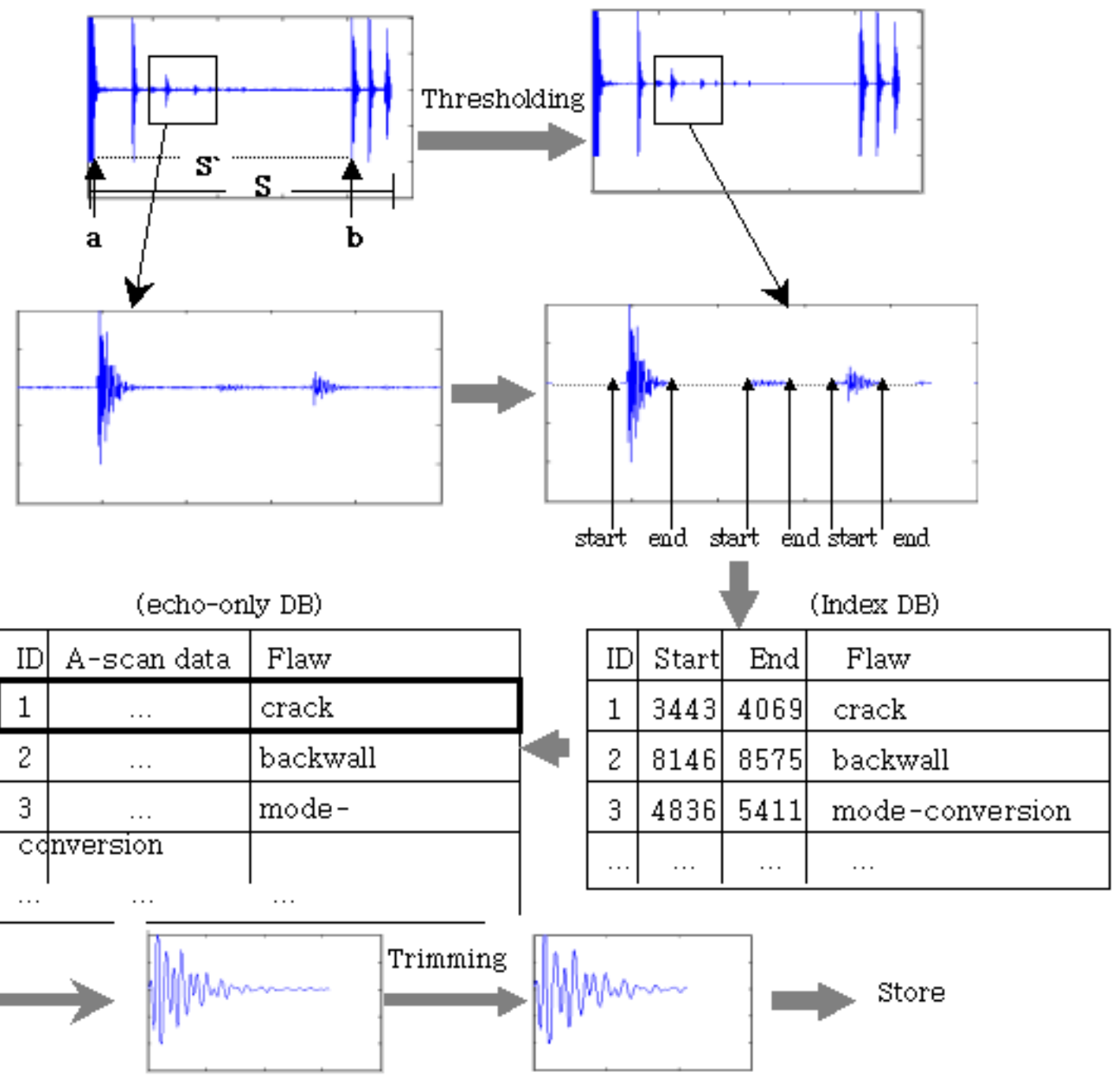

Figure 5.4: The flow-chart for the method to decide echo borders.

The gating method proposed in this chapter has the following steps. 
1. Capture A-scan signals (the time period must be long enough to include an initial pulse (IP) and at least one back-wall echo (BE)). We denote the captured A-scan signal vector by $S=(1,2, \cdots, m)$ where $m$ is the total size of the A-scan.

2. Search the position of maximum amplitude including the IP and the BE. The maximum amplitude found within the first half part of S is considered as IP. In the same way, find the maximum amplitude within the last half part of $\mathrm{S}$ and consider its position as BE. If more than one maximum amplitude exists for each half range, choose the rightmost point as IP and the leftmost point as BE. Let the searched position for $\mathrm{IP}$ and BE be $a$ and $b$ respectively, then a signal section between $a$ and $b$ inclusively is $S^{\prime}=(a, a+1, a+2, \cdots, b)$.

3. Calculate the thresholding parameter that is to be used for filtering noise from the original A-scan data. One method that is suitable for DWT is chosen and applied. The method determines a thresholding parameter thr as follows:

$$
t h r=\sqrt{2 \times \log (n)} \times c,
$$

where $c$ is an estimate of level noise and $n$ is equal to $\left\|S^{\prime}\right\|$ (the length of the signal between IP and BE).

4. Threshold the originally captured A-scan $S$ with the thresholding parameter thr and let the thresholded A-scan be $t h \_S$.

5. Scan $t h \_S$ and if more than 50 continuous zeros are present, assume two separate echoes to exist before and after the sequence of zeros. Store the start point and end point of each separated echo. If the purpose is to construct the training data sets for a classifier, the human expert must also record whether the echo originates from a crack, mode-conversion or the back-wall. These stored records are named "index DB" in Figure 5.4 and become an index for the next step.

6. Construct a database of signal echoes which have original A-scan data between the start point and end point corresponding to the index DB constructed at Step 5.

7. Trim the long head or tail with low amplitude of each signal echo. Assuming that a trimming rate is set to $x$ (measured as a percentage), each signal echo is reduced from its edge until the total amount of energy contained in the removed edge values 
reaches $x \%$ of the total energy of the signal echo. Then construct final records of the trimmed signal echoes and their corresponding flaw types. These finally stored signal echoes become a base for further feature extraction.

\subsection{Experiment}

\subsubsection{Experimental setting}

The experimental setting consisted of two main processes. Firstly, signal segments of interest from the whole ultrasonic A-scan signals were selected using five different schemes. These five schemes were Random positioning (RA), Central peak positioning (CP), Systematic echo capturing and preservation of original neighboring grass (SO), Systematic echo capturing method with zero-padding (SZ) and Main energy computing (ME). The detailed description of each method is in the next subsection. On the signal data preprocessed by five different methods, we applied two different feature extraction techniques (DWT versus CDWT) to extract more informative features from the selected signal features. The next step of the overall experiment was to construct ANN-based classifiers and SVM-based classifiers trained by five different feature sets.

For the initial data collection, we acquired A-scan signals from a number of shafts with various geometries and lengths. To construct the database for the experiment, signal echoes were captured and segmented by the five different schemes. Whether the signal originated from a crack, mode-conversion or back-wall was also recorded. Once we had built an initial database holding the corresponding five different schemes, we mapped the vectors in the database to the feature domain based on two different feature extraction techniques (the DWT and the CDWT), generating ten different sets of training data. We used these ten sets of training data for training respective ANN models and SVM models and we validated classification performance through cross-validation. A detailed description of the experiment follows. 


\subsubsection{Preprocessing and Feature Extraction}

A-scan signals were acquired from seven shafts by using $2 \mathrm{MHz}$ frequency. A training data set with 43 crack echoes, 55 mode-converted echoes and 52 back-wall echoes was used. The main purpose of this experiment was to investigate if the newly developed method for gating a signal section and singling out an echo plays an effective role in overcoming the time-variance problems of DWT. For this purpose, five different schemes for the selection of the signal's region of interest were applied and compared. These are listed as follows.

\section{Random positioning (RA).}

No special rule is applied in the way of capturing. The time window (gate) segments of A-scan have a regular size so as to include the EXTENT of an echo and, therefore, its point of highest amplitude. However, it may pick parts of other echoes or a large proportion of data before or after the echo. For the experiments reported in this paper, 768 data points were segmented for each pattern and then down-sampled into 256 points before the conversion to DWT coefficients in order to reduce the dimension required in the $\mathrm{ANN}$.

2. Central Peak positioning (CP).

This is a typically used rule in many other ultrasonic signal tests. This extracts a signal section in which the signal peak is positioned in the center. As mentioned in Section 5.2, this rule can be problematic when applied to signal echoes with skewed energy distribution. In the same way as RA, we produced input vectors of length 256 for further processing; that is, we applied the same level of down-sampling.

3. Systematical echo capturing method with preservation of original neighboring grass (SO).

The start point $(s)$ and the end point $(e)$ of echo-only sections were decided by using the method described in Section 5.3. Then signal segments which start from the point $s$ to $(s+767)$ on the original A-scan signal were extracted and finally down-sampled to 256. The position for gating was decided systematically but unnecessary neighboring noised section may be included.

4. Systematical echo capturing method with zero-padding (SZ). 
The border of the echo-only section is decided in the same way as $\mathbf{S O}$, but the difference from SO is not to attach the background neighbor section but to perform zero-padding when the size of the selected echo-only section is smaller than 768 . In the same way as other methods, the 768 points signal data was down-sampled for the next step in order to serve as input into the classifier. The main property of this method is that the neighboring background grass section is excluded as much as possible, thus the background noise has less effect on the signal classification.

5. Main energy capturing (ME).

After the echo-only area was extracted, 256 signal points were selected starting from the central point in terms of energy distribution.

The next step converted each of these five sets of time domain data into different domain feature sets which would later become the input vectors to the ANNs or SVMs. For this purpose, the time domain data selected by the various schemes explained above were all transformed into the DWT coefficients using Daubechies wavelets [26]. Note that we presented a detailed description of this transform in Section 2.4.

The same experimental process was also performed using Clustered-DWT (CDWT) [73]. This technique was suggested as a potential approach to overcome the time-variance problem that DWT coefficients have. CDWT uses the energy of the different bands to cluster the coefficients. In particular, the unsupervised learning method clusters the coefficients on the basis of the average energies at different scales and within the same scale. The Euclidian norms of clusters are used as features for the classification.

We produced five time-domain pattern sets. We converted signals through five different schemes (RA, CP, SO, SZ and ME) so that a vector of 256 sample points constituted the data vectors for classification. Note that these are still long signal patterns of DWT features. We compressed these DWT coefficients into 128 values by discarding the last 128 coefficients which do not contain as much information but mainly noise. We stored these vectors of length 128 and with DWT coefficients as DWT features to build the feature set. These vectors of DWT coefficients constitute feature sets that we further mapped to cluster energy features by the clustering method [73], and which also became CDWT feature sets. As the number of clusters for five pattern sets were all different, the largest number of 
clusters being 64 , we needed to add extra zeros at the end of other smaller dimensional feature sets to homogenize the dimension to 64 .

\subsubsection{ANN Classification}

For the construction of the ANN model, fully connected feed-forward neural networks with two hidden layers were used for classifying the shaft signals into cracks, mode-conversion or back-wall. We trained them using the back-propagation algorithm in batch mode. As there are two typed feature vectors (the 128 dimensional DWT feature vectors and the 64 dimensional clustered-DWT vectors) using five different preprocessing schemes, we designed two different neural network architectures having 128 and 64 input nodes, respectively. For the DWT feature sets, an ANN architecture was designed having two hidden layers with 64 nodes and 16 nodes and an output layer with 3 nodes. The ANN for clustered-DWT had 16 and 8 nodes in two hidden layers and same types of output nodes as DWT. We set the learning rate to 0.2 and we applied topological order as the update mode of the networks.

We shuffled samples and randomly divided them up into 10 sets. In turn, we used 9 of these to train a network and we used the remaining set to validate the network. We repeated the procedure with all ten possible combinations, and furthermore, we repeated the process 10 times to obtain an average of the network training ability by assigning 10 different sets of initial weights to the network. We carried out this 10-fold cross-validation test on ten ANNs trained with ten feature sets: five DWT-feature sets and five clustered-DWT-feature sets. All of these five different variants corresponded to the five different schemes (RA, CP, $\mathrm{SO}, \mathrm{SZ}$ and ME). We compared the performance of ten different schemes using the result of each cross-validation test.

The Mean Square Error (MSE) limit was set to 0.001 for stopping the training process, which resulted in efficient training. The epoch limit was set to 200000 for those occasional cases where training failed to converge. To overcome the over-fitting problem during the process of training ANNs, the error over the validation data was monitored and the training was forced to stop on the epoch that produced the lowest error over the validation data. We plotted the MSE, both over the training data and over the validation data in every training process. The monitoring was based on this plot which displayed how the MSE varied with 
the number of epochs. The point of lowest error on the validation set is difficult to identify without this plot.

\subsubsection{SVM classification}

As mentioned in Chapter 4, SVM has gained wide acceptance as a powerful supervised learning algorithm based on statistical learning theory. The remarkably robust performance of SVM is also shown in Chapter 4 through some experiments. Therefore, in addition to ANN models, we used SVM models in order to investigate whether the new method for selecting the horizontal region of interest introduced in Section 5.3 outperformed other generally used methods for extracting features in ultrasonic signals from shafts.

For the experiment reported in this chapter, we employed RBF kernels for constructing SVM classifiers. The RBF kernel approach provides nonlinear mapping, requires comparatively small numbers of hyper-parameters and has fewer numerical difficulties. Two parameters: a penalty parameter $C$ and kernel parameter $\gamma$ are required for RBF kernels. Among several previously suggested methodologies for searching the best parameters for designing SVM models [34, 46, 84], a grid searching algorithm [37] was used in this experiment. Using this method, pairs of $C$ and $\gamma$ were tried and we picked the one with the best 10-fold-crossvalidation accuracy. This method of searching for parameters is the same as presented in Chapter 4 and the detail of the grid searching method we used is described in Section 4.3.3. The results of grid searching for 10 different groups of training data (DWT-RA, DWTCP, DWT-ME, DWT-SO, DWT-SZ, CDWT-RA, CDWT-CP, CDWT-ME, CDWT-SO and CDWT-SZ) are graphically summarized in Appendix B. Following this method, we set $C$ and $\gamma$ for ten different sets of training patterns as shown on Table 5.1

In the same way as the comparison test using ANN classification, we carried out 10-fold cross-validation tests on ten SVM models, which we trained through ten feature sets. We compared classification accuracy (performance) of those different schemes using the result of each cross-validation test. 
Table 5.1: Parameter setting for SVM models.

\begin{tabular}{|c|c|c|c|c|}
\hline & \multicolumn{2}{|c|}{ DWT } & \multicolumn{2}{|c|}{$\begin{array}{c}\text { Clustered DWT } \\
(\text { CDWT })\end{array}$} \\
\hline & $C$ & $\gamma$ & $C$ & $\gamma$ \\
\hline RA & 1 & 0.125 & 8 & 0.5 \\
\hline CP & 32 & 0.03125 & 128 & 0.0078125 \\
\hline ME & 1 & 0.03125 & 32 & 0.00049 \\
\hline SO & 1 & 0.125 & 32 & 0.125 \\
\hline $\mathrm{SZ}$ & 8 & 0.03125 & 1 & 0.0078125 \\
\hline
\end{tabular}

\subsection{Results and Analysis}

We compared the results of feature extraction algorithms using 10-fold cross-validation tests (a total of one hundred tests for each network configuration). We summarized the results using percentage of accuracy in Table 5.5. Figure 5.5 offers a visual comparison between the classification performance of the ten different feature extraction schemes. This picture shows histograms presenting relative values of the results shown in Table 5.5. The statistical significance of those test results is validated through ANOVA test $(\mathrm{p}=0.05)$ which is summarized in Table 5.5. The full test results are listed in Appendix A.3.

The following points are noteworthy based on the results of the experiment.

- As seen through the classification result (Table 5.5) and its ANOVA test result (Table 5.5), different preprocessing methods affected the classification result of every types of classifier (ANN-DWT, ANN-CDWT, SVM-DWT or SVM-CDWT).

- Among the five suggested preprocessing methods, the RA method showed the worst classification performance on both classifier models (ANNs and SVMs). This result confirms the well known time-variance problem of DWT coefficients. In contrast, the comparatively high performance of $\mathrm{CP}, \mathrm{SO}$ and $\mathrm{SZ}$ indicates that applying a systematic rule for deciding a signal's section of interest is effective to overcome the time-variance problem.

- The performance result for the CP method shows that this systematic rule still deserves to be popularly used. However, the performance can be improved by using a 
method such as SO or SZ by isolating out the echo section.

- SZ is clearly at the top among the five different schemes. This result implies that use of echo sections that exclude their neighboring grass sections, brings out a positive effect. In particular, using the SZ method reduces the risk of not being detected among the background noise. This result also shows that there is potential in the reduction of features (and thus, classifier's dimension).

- The relatively low performance of ME implies that the signal sections extracted based on the central position of energy value do not always encapsulate the most important time-domain signal features. Most shaft signal flaw echoes cannot be approximated by well-defined bell curves, so in this case, the central energy positioning is somewhat meaningless.

- There are not a significant difference in the classification performance between classifiers using DWT and those using CDWT. Table 5.5 presents that the statistical significance (between DWT and CDWT) exists only on the result of ANN-RA, ANNSO and SVM-RA among 10 different possible types of classifiers. Considering that the superiority of CDWT to DWT has been proved by other previous study [73], our experimental result implies that the new preprocessing methods help to construct more improved sets of DWT patterns.

\subsection{Remarks}

In this chapter, we introduced a new technique to derive a preprocessing method for timedomain A-scans signals. This technique offers consistent extraction of a segment of the signal from long signals that occur in the NDT of shafts. We compared and evaluated using two different classifiers namely ANNs and SVMs. We achieved this by supplying feature sets generated with our new preprocessing method and recording their classification performance over repeated cross-validation. Their classification performance was also compared and evaluated with other alternative techniques and this investigation established experimentally that DWT coefficients can be used as a feature extraction scheme more reliably by using our new preprocessing technique. The analysis of the experiment results shows a rather 
Table 5.2: Classification performance of DWT and clustered-DWT feature extraction schemes using five different preprocessing methods

\begin{tabular}{|c|c|c|c|c|c|c|c|c|c|c|}
\hline & \multicolumn{10}{|c|}{ ANN } \\
\hline & \multicolumn{5}{|c|}{ DWT } & \multicolumn{5}{|c|}{ CDWT } \\
\hline & $\mathbf{R A}$ & $\mathbf{C P}$ & ME & SO & SZ & RA & $\mathbf{C P}$ & ME & SO & SZ \\
\hline Test 1 & 48.4 & 81.1 & 78.9 & 77.6 & 89.5 & 73.6 & 81.1 & 74.8 & 89 & 87.6 \\
\hline Test 2 & 53.7 & 85.3 & 76.8 & 84.2 & 86.8 & 74.4 & 82.1 & 70.4 & 80 & 91.8 \\
\hline Test 3 & 56.8 & 85.3 & 72.6 & 76.3 & 86.8 & 69.4 & 83.4 & 72.6 & 82.8 & 87.6 \\
\hline Test 4 & 53.7 & 82.1 & 72.6 & 76.3 & 85.5 & 76.8 & 87.2 & 70.8 & 89 & 81.6 \\
\hline Test 5 & 48.4 & 80 & 76.8 & 73.7 & 75.8 & 71.8 & 83 & 80 & 80.3 & 86.3 \\
\hline Test 6 & 63.2 & 100 & 63.2 & 82.1 & 82.1 & 82.8 & 81.6 & 70.4 & 88.8 & 88.8 \\
\hline Test 7 & 48.4 & 69.5 & 88.4 & 72.6 & 100 & 76.8 & 94.8 & 78.4 & 81.6 & 91.8 \\
\hline Test 8 & 63.2 & 72.6 & 72.6 & 88.4 & 86.8 & 56.8 & 88.8 & 79.7 & 91.8 & 88.8 \\
\hline Test 9 & 50.5 & 94.7 & 88.4 & 72.6 & 92.9 & 75.6 & 75.6 & 63.2 & 80 & 84.2 \\
\hline Test 10 & 37.6 & 75.9 & 69.5 & 72.6 & 89.5 & 69.6 & 81.6 & 76.8 & 87.6 & 94.8 \\
\hline \multirow[t]{4}{*}{ Average } & 52.4 & 82.7 & 76 & 77.6 & 87.6 & 72.8 & 83.9 & 73.7 & 85.1 & 88.3 \\
\hline & \multicolumn{10}{|c|}{ SVM } \\
\hline & \multicolumn{5}{|c|}{ DWT } & \multicolumn{5}{|c|}{ CDWT } \\
\hline & $\mathbf{R A}$ & $\mathbf{C P}$ & ME & SO & SZ & RA & $\mathbf{C P}$ & ME & SO & SZ \\
\hline Test 1 & 68.8 & 82.6 & 87.5 & 96.2 & 96.2 & 68.8 & 87.5 & 93.8 & 87.5 & 89.4 \\
\hline Test 2 & 75 & 87.5 & 75 & 93.8 & 87.5 & 87.5 & 82.6 & 68.8 & 96.8 & 90.6 \\
\hline Test 3 & 68.8 & 75.7 & 75 & 93.8 & 93.8 & 75 & 68.8 & 75 & 93.8 & 93.8 \\
\hline Test 4 & 60 & 100 & 86.7 & 84.7 & 100 & 73.3 & 93.3 & 93.3 & 93.3 & 86.7 \\
\hline Test 5 & 81.3 & 100 & 93.8 & 86.7 & 97.2 & 81.3 & 93.8 & 93.8 & 83.2 & 83.2 \\
\hline Test 6 & 69 & 86.9 & 75.6 & 94.2 & 94.8 & 69 & 94.8 & 75.6 & 94.8 & 96.9 \\
\hline Test 7 & 68 & 92.4 & 79 & 89.3 & 92.4 & 74.8 & 74.2 & 88.2 & 92.4 & 92.4 \\
\hline Test 8 & 74.8 & 89.5 & 81.4 & 92.4 & 88.8 & 88.8 & 83.3 & 85.4 & 92.4 & 96.1 \\
\hline Test 9 & 69.6 & 86.9 & 86.9 & 89 & 86.9 & 86.9 & 86.9 & 85.2 & 86.9 & 96.9 \\
\hline Test 10 & 79.4 & 92.4 & 82.6 & 96.2 & 92.4 & 94.6 & 96.2 & 92.4 & 96.2 & 89.6 \\
\hline Average & 71.5 & 89.4 & 82.3 & 91.6 & 93 & 80 & 86.1 & 85.1 & 91.7 & 91.6 \\
\hline
\end{tabular}




\begin{tabular}{|c|c|c|c|c|c|c|c|}
\hline \multirow{2}{*}{$\begin{array}{l}\text { Preprocessing } \\
\text { method }\end{array}$} & \multirow{2}{*}{ Classifier } & \multirow{2}{*}{$\begin{array}{c}\text { Feature } \\
\text { extraction } \\
\text { scheme }\end{array}$} & \multicolumn{5}{|c|}{ relative values of classification accuracy } \\
\hline & & & 0.2 & 0.4 & 0.6 & 0.8 & 1 \\
\hline \multirow{4}{*}{ SZ } & \multirow{2}{*}{ SVM } & CDWT & & & & & \\
\hline & & DWT & & & & & \\
\hline & \multirow{2}{*}{ ANN } & CDWT & & & & & \\
\hline & & DWT & & & & & \\
\hline & & & & & & & \\
\hline \multirow{4}{*}{$\mathrm{SO}$} & \multirow{2}{*}{ SVM } & CDWT & & & & & \\
\hline & & DWT & & & & & \\
\hline & \multirow{2}{*}{$\mathbf{A N N}$} & CDWT & & & & & \\
\hline & & DWT & & & & & \\
\hline & & & & & & & \\
\hline \multirow{4}{*}{ ME } & \multirow{2}{*}{ SVM } & CDWT & & & & & \\
\hline & & DWT & & & & & \\
\hline & \multirow{2}{*}{$\mathbf{A N N}$} & CDWT & & & & & \\
\hline & & DWT & & & & & \\
\hline \multirow{4}{*}{$\mathbf{C P}$} & \multirow[b]{2}{*}{ SVM } & CDWT & & & & & \\
\hline & & DWT & & & & & \\
\hline & \multirow{2}{*}{ ANN } & CDWT & & & & & \\
\hline & & DWT & & & & & \\
\hline & & & & & & & \\
\hline \multirow{4}{*}{$\mathbf{R A}$} & \multirow{2}{*}{ SVM } & CDWT & & & & & \\
\hline & & DWT & & & & & \\
\hline & \multirow{2}{*}{ ANN } & CDWT & & & & & \\
\hline & & DWT & & & & & \\
\hline
\end{tabular}

Figure 5.5: Comparison of performance of different feature extraction techniques. 
Table 5.3: A summarized result of the ANOVA test conducted for the results presented in Table 5.5

\begin{tabular}{|c|c|c|c|c|}
\hline \multicolumn{3}{|c|}{ Groups } & \multicolumn{2}{|r|}{ F-test } \\
\hline Classifier & $\begin{array}{c}\text { Feature Extraction } \\
\text { Method }\end{array}$ & $\begin{array}{c}\text { Preprocessing } \\
\text { Method }\end{array}$ & F value & $\begin{array}{c}\text { F-test } \\
\text { Critical Value }\end{array}$ \\
\hline \multirow{2}{*}{$\mathrm{ANN}$} & DWT & \multirow{4}{*}{$\mathrm{RA}, \mathrm{CP}, \mathrm{ME}, \mathrm{SO}, \mathrm{SZ}$} & 32.92 & \multirow{4}{*}{2.579} \\
\hline & CDWT & & 18.13 & \\
\hline \multirow{2}{*}{ SVM } & DWT & & 23.44 & \\
\hline & CDWT & & 4.16 & \\
\hline \multirow{5}{*}{$\mathrm{ANN}$} & \multirow{10}{*}{ DWT, CDWT } & $\mathrm{RA}$ & 39.37 & \multirow{20}{*}{4.414} \\
\hline & & $\mathrm{CP}$ & 0.14 & \\
\hline & & $\mathrm{ME}$ & 0.57 & \\
\hline & & $\mathrm{SO}$ & 10.82 & \\
\hline & & SZ & 0.10 & \\
\hline \multirow{5}{*}{ SVM } & & $\mathrm{RA}$ & 6.01 & \\
\hline & & $\mathrm{CP}$ & 0.76 & \\
\hline & & $\mathrm{ME}$ & 0.64 & \\
\hline & & $\mathrm{SO}$ & 0.001 & \\
\hline & & $\mathrm{SZ}$ & 0.53 & \\
\hline \multirow{10}{*}{$\mathrm{ANN}, \mathrm{SVM}$} & \multirow{5}{*}{ DWT } & RA & 37.29 & \\
\hline & & $\mathrm{CP}$ & 3.19 & \\
\hline & & $\mathrm{ME}$ & 3.96 & \\
\hline & & $\mathrm{SO}$ & 42.17 & \\
\hline & & $\mathrm{SZ}$ & 4.99 & \\
\hline & \multirow{5}{*}{ CDWT } & $\mathrm{RA}$ & 4.06 & \\
\hline & & $\mathrm{CP}$ & 0.44 & \\
\hline & & $\mathrm{ME}$ & 11.87 & \\
\hline & & $\mathrm{SO}$ & 10.86 & \\
\hline & & $\mathrm{SZ}$ & 2.93 & \\
\hline
\end{tabular}


large set of evidence that the new approach to extracting a meaningful section for an echo among a large A-scan is effective in obtaining information that can be converted robustly by DWT into features for classification by ANNs or SVMs. 


\section{Chapter 6}

\section{Ensembles for Ultrasonic Signal Classifiers}

\subsection{Introduction}

In order to enhance the classification performance more accurately and reliably, two main issues are to identify the better set of features and to identify the more suitable learning algorithm. As presented in previous chapters of this thesis, our research has aimed at selecting better sets of features with physical significance (Refer to Chapter 2 and Chapter 5) or exploring candidate classifiers (Refer to Chapter 3 and Chapter 4) for ultrasonic shaft signal classification. The most competitive feature extraction approaches are Fast Fourier Transform (FFT) and Discrete Wavelet Transform (DWT). Artificial Neural Networks (ANN) and Support Vector Machines (SVM) are the top two approaches to build classifiers in this field. Therefore, we focussed on finding the best single classifier model (between ANN and SVM) and determining the best-selected feature extraction scheme (between FFT and DWT).

In this chapter, however, we learn multiple models from the shaft test data and combine their outputs for making a final decision for classification. Figure 6.1 graphically shows the difference between two methods of constructing an AUSC system using two feature 
extraction schemes (FFT and DWT) and two learning algorithms (SVM and ANN). The system Type I is constructed by combining decisions from multi-classifiers and the system Type II is by choosing the best single classifier among multi-classifiers. The motivation for the ensemble of classifiers (Type I in Figure 6.1), rather than choosing the best single classifier (Type II in Figure 6.1) was that the FFT might reflect physical properties that are different from those the DWT conveys. We suspected that including the FFT as another informant of the decision process, even if the accuracy using the DWT was shown to be superior, should improve accuracy. If we only relied on the system Type II, we would totally dismiss the outcomes of a classifier trained by an FFT feature set as the accuracy using the DWT has been shown to be superior.

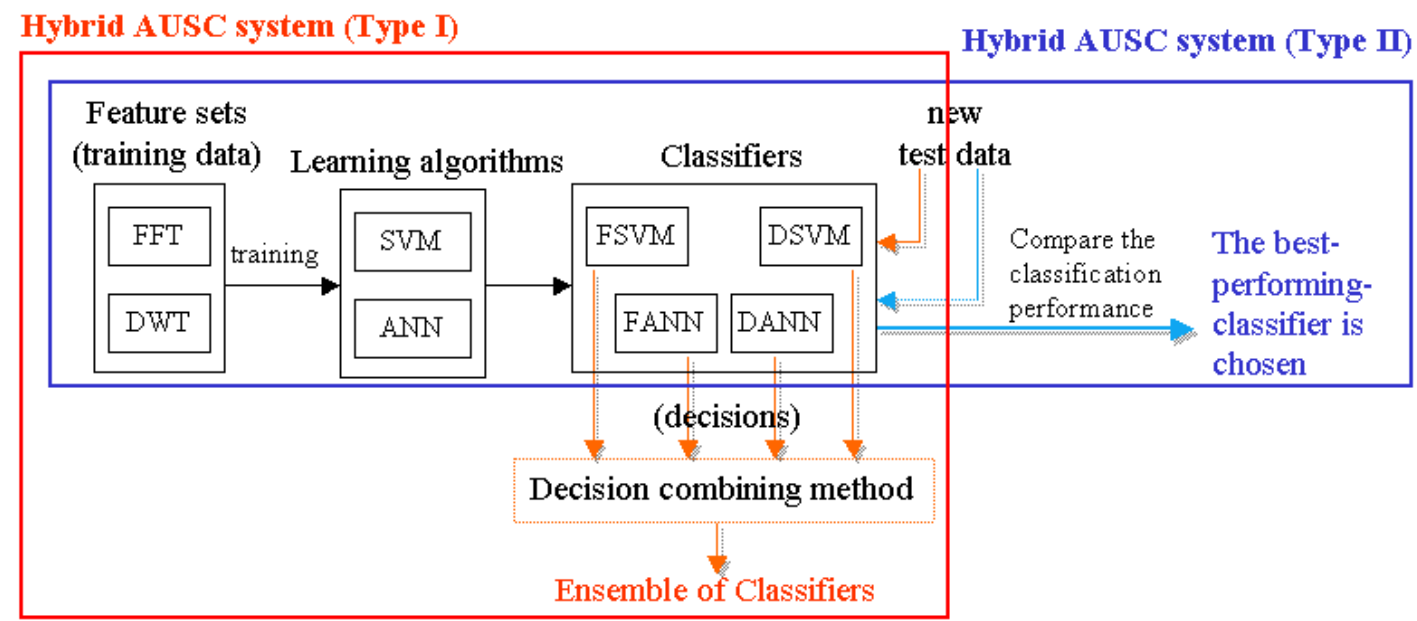

Figure 6.1: Constructing methods of two different AUSC systems using two feature extraction schemes (FFT and DWT) and two learning algorithms (SVM and ANN): The system Type I is constructed by combining decisions from multi-classifiers and the system Type II is by choosing the best classifier among multi-classifiers.

This chapter aims to confirm the observation that there seem to be uncorrelated errors among the variants explored in previous chapters, and therefore an ensemble of classifiers will achieve better discrimination accuracy. We explore the diverse possibilities of heterogeneous and homogeneous ensembles, combination techniques, feature extraction methods and classifier types and provide guidelines to which combinations result in superior performance. We also explore the effect of combining multiple models not only on the overall classification performance but also on classifying each class. The analysis on the investiga- 
tion results obtained becomes the basis for the construction of an integrated multi-classifier model using both feature extraction schemes (FFT and DWT) effectively.

Our presentation continues in Section 6.2 with a brief summary of previous studies for "combining classifiers" and also provides a motivation of observations for attempting ensembles of classifiers for improving classification systems for shaft test data. Section 6.3 describes our empirical evaluation, including the description of how we generated and combined multiple models and how we evaluated the classification performance of each ensemble. Section 6.4 analyzes the experimental result from various combination schemes and compares and discusses their performance. Conclusions of these experiments appear in Section 6.5.

\subsection{Combining Multi-Classifiers}

Combining classifiers improves the accuracy achieved by a single classifier when different classifiers implicitly represent different useful aspects of the input data [25, 29, 36]. Techniques for combining multiple classifiers must solve two issues: 1) how to generate multiple models (model generation) and 2) how to combine the prediction of the multiple models to produce an overall classification (model combination). There have been various approaches for model generation and for model combination [29, 31, 98].

We can summarize two different approaches for generating multiple models. Figure 6.2 presents the procedure of those methods of model generation. One approach is applying a single algorithm repeatedly to different versions of the training data (homogeneous approach). For the homogeneous approach, there are two ways of manipulating different versions of the training data: either by different feature extraction schemes [71] or by partitioning a data set into multiple sets $[9,10]$. The other approach for generating multiple models is applying different learning algorithms to the same data to build multiple classifiers (heterogeneous approach) [4]. There are also various techniques for combining the predictions obtained from the multiple classifiers, such as voting, stacking and cascading. ${ }^{1}$

\footnotetext{
${ }^{1}$ We will not discuss those methods further in this chapter, because this chapter mainly focuses on seeking for the most suitable model-generating paradigm using two feature extraction schemes (FFT and DWT) and two learning algorithms (ANN and SVM) for the ensemble approach for ultrasonic shaft signal classification.
} 

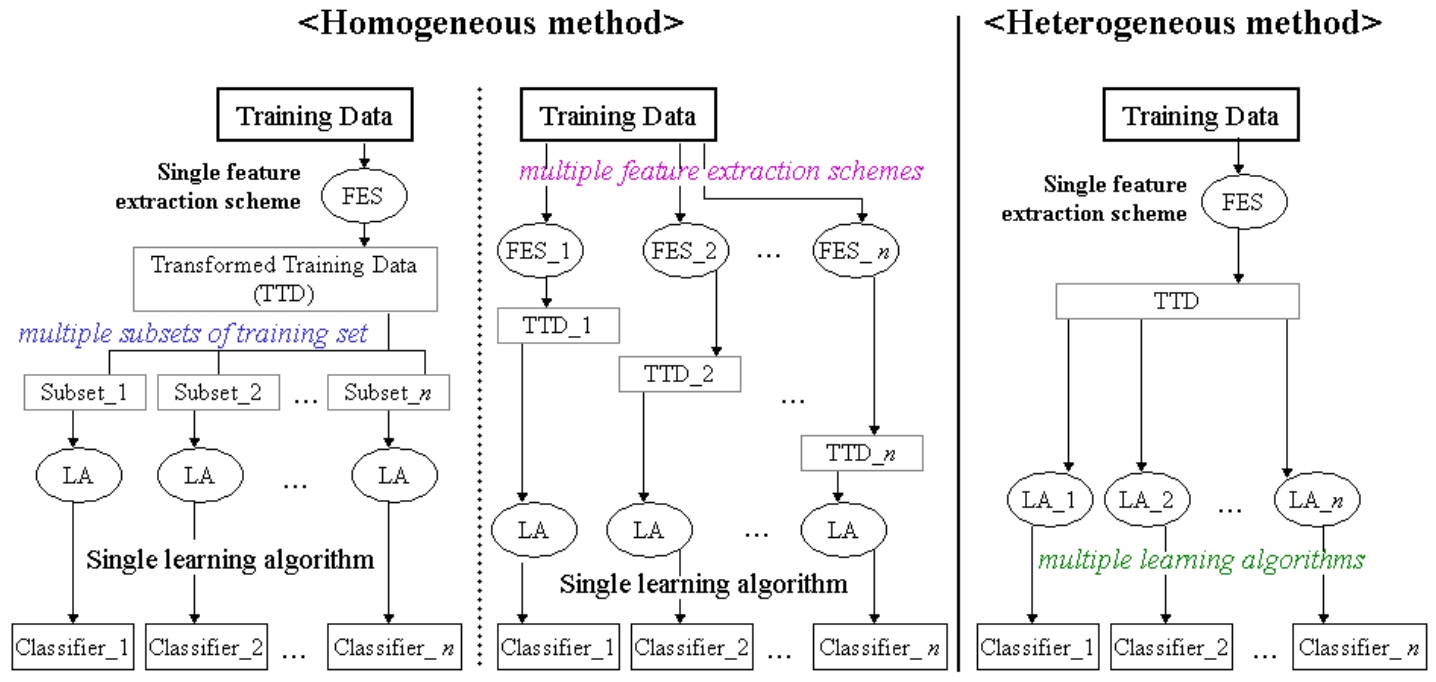

Figure 6.2: Methods of generating multi-classifiers for ensembles.

Therefore, in order to construct an effective multi-classifier system, we need to decide a scheme for model generation as well as a combination method for decision-making. Since we design an ensemble of models using two different feature sets, constructed by FFT and by DWT, we will not apply such schemes as boosting or bagging $[9,32]$ where only one feature extraction scheme is used. The diversity of techniques for generating and combining models raises the issue of which method for generating and combining multiple models to choose for constructing the most effective and reliable multi-model systems for our application domain. The theory suggests generating a set of models that are diverse in that they make errors in different ways $[41,54,102]$.

Through this chapter, we wish to investigate the classification performance by multi-models. Is better performance obtained when participant classifiers are trained by FFT features or DWT features? We also generate multiple models using different learning algorithms (SVM and ANN), and decide which combination paradigm is more suitable. Therefore, in this chapter, we report results of our investigation into which method for model generation offers more improvement on the accuracy achieved by a single classifier. We generate heterogeneous and homogeneous models and combine the outputs of those multi-classifiers by three widely known combining techniques: Bayesian Combination (BC), Distribution Summation (DS) and Likelihood Combination (LC). We analyze the types of errors on each combina- 
tion model in order to gain insight into appropriate combining strategies. The details of our investigation follow in Section 6.3.

\subsection{Experiment}

The whole experimental setting consists of the following steps:

1. We first mapped shaft inspection data into feature domains using two feature extraction schemes (FFT and DWT).

2. We trained them through SVM models and ANN models using 5-fold cross-validation learning and recorded their performance as single models.

3. We combined single models across two dimensions: 1) combining the decisions of the FFT model and the DWT model trained by a single learning paradigm and 2) combining the decisions of the SVM model and the ANN model with the same feature scheme. We applied 3 combining methods for each combination.

4. We compared the classification accuracy results from the three combined models with the results of using a single model.

Note that the first and second steps constitute a procedure for generating multiple models. The third step is the procedure for combining those multiple models. Figure 6.3 graphically summarizes those two procedures (model generation and model combination) of our experiment. Figure 6.4 presents the whole procedure in our experiment and the details are presented in following subsections.

\subsubsection{Generating multiple classifiers}

The procedure for the construction of a database for the experiment is similar to those we used in other experiments for previous chapters of this thesis. That is, we acquired A-scan signals from eight various shafts, ranging between $100 \mathrm{~mm}$ and $1300 \mathrm{~mm}$ in length, using 


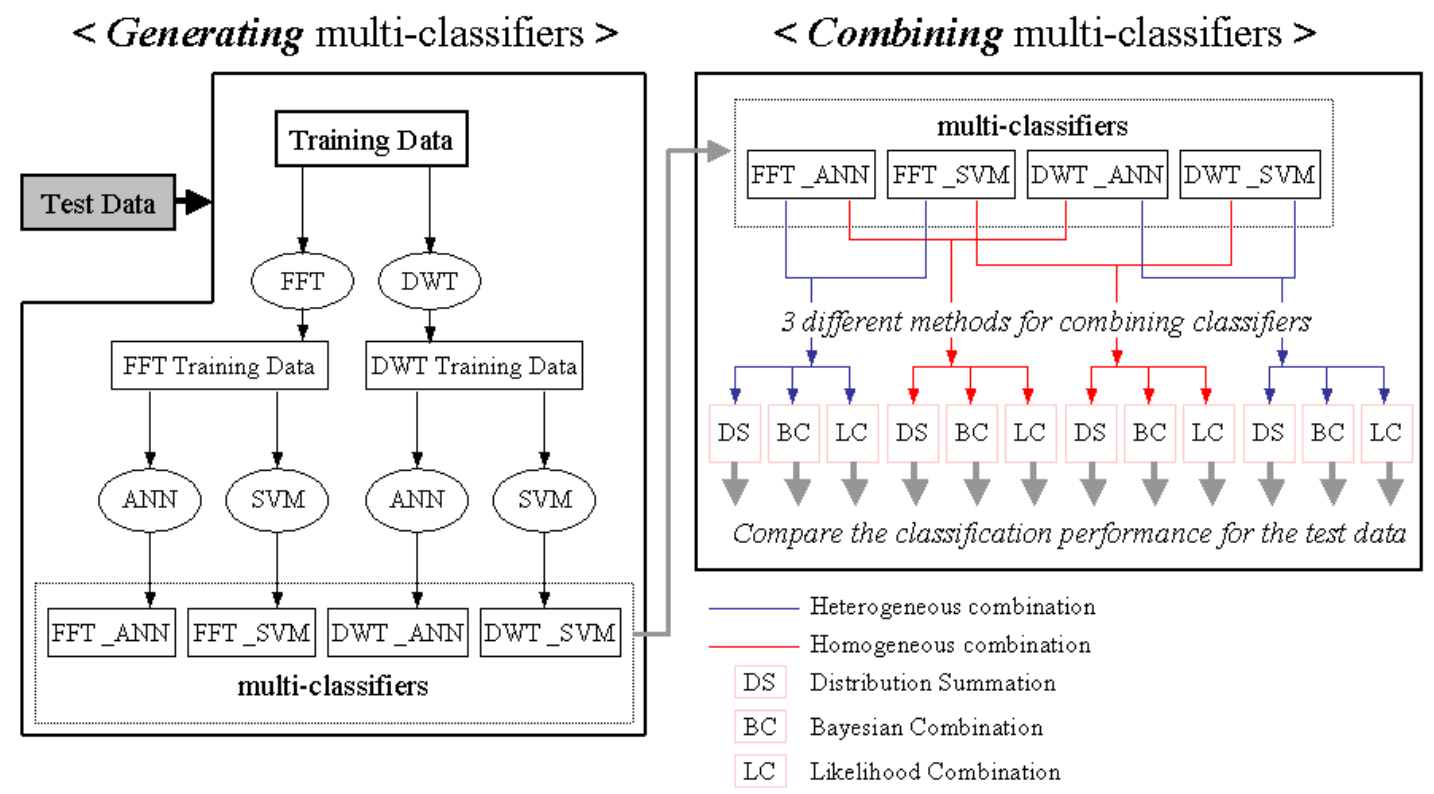

Figure 6.3: Diagram of our experiment presenting two main procedures: model-generation and model-combination.

a probe with frequency set to $2 \mathrm{MHz}$. Five of these shafts contained cracks and the rest were clean. Mode-converted echoes were captured from these shafts, except for one $100 \mathrm{~mm}$ calibration block that did not produce mode-converted echoes because of its geometry. We extracted more than 1000 signal segments of interest from the whole ultrasonic Ascan signals. We also recorded whether the echoes were caused by Crack (CR), ModeConversion (MC) or Design Features (DF). As mentioned before, one of the issues of concern in ultrasonic shaft inspection is that the signal echoes caused by CR can be confused by fainted echoes caused by MC and vice versa. For our experiment in this chapter, we chose 60 signals randomly from $\mathrm{CR}$ data as well as 60 random signals from $\mathrm{MC}$ data among the sample pool. Thus, a data set with a total of 120 time-domain signals became the basis for further processing.

In order to apply a consistent way of signal segmentation, which is necessary for suppressing time-variance problems with the DWT, we used a systematic echo capturing method with zero padding (SZ). This preprocessing technique has been introduced and described in Chapter 5. Using this gating method, we captured the 768 values of long time-domain vectors, and down-sampled them into 128 values for input into the FFT. We concatenated 


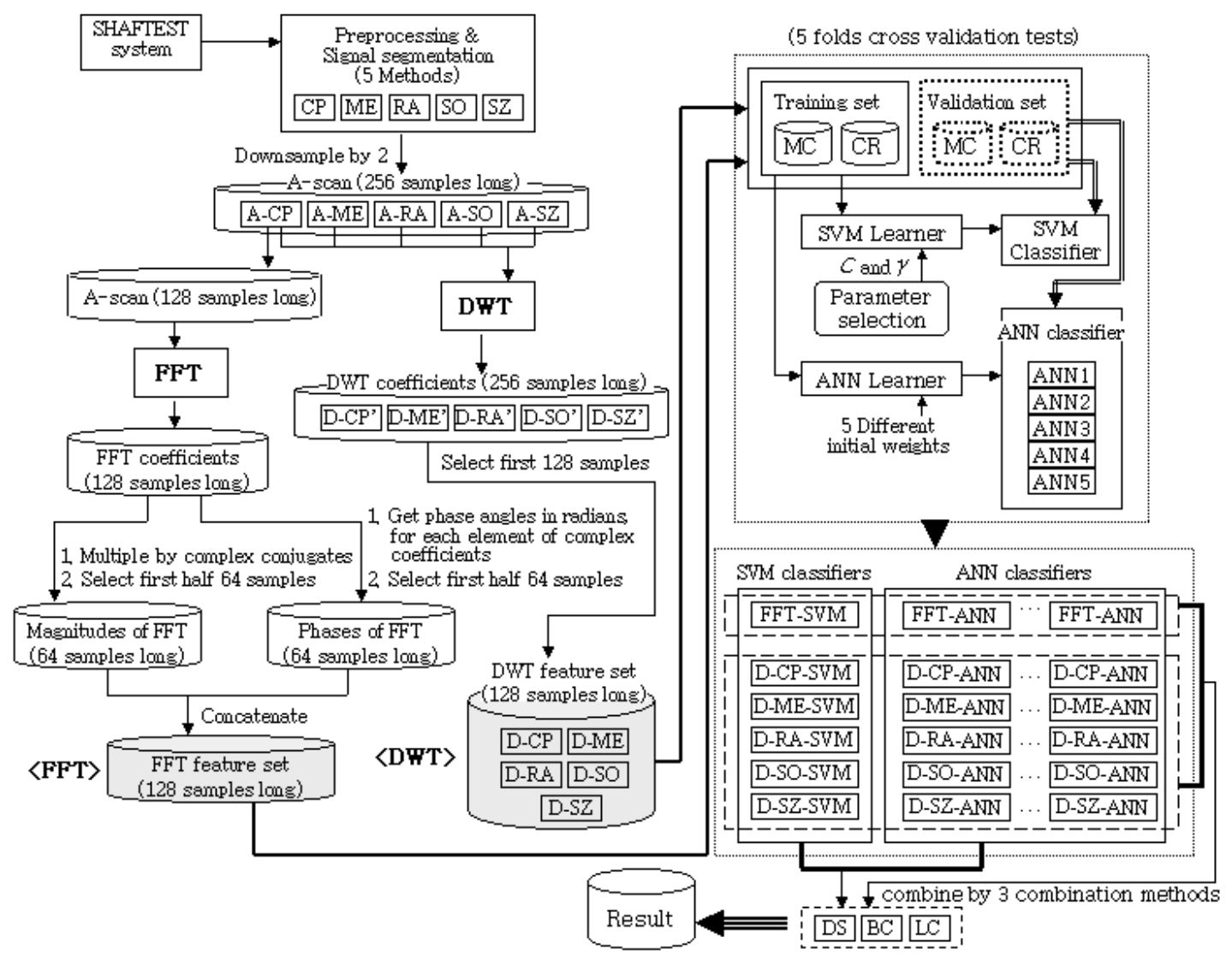

Figure 6.4: Overall procedure of our experiment for building an ensemble of classifiers. 
the sequences of magnitude components and phase components (FFT coefficients) into 128 dimensional pattern vector for classification. In parallel, we compressed the 768 values representing the DWT coefficients into 128 samples by discarding the last 128 coefficients (supposing them not to contain much information but mainly noise). We stored the 128 long vectors of DWT coefficients as the DWT feature set. For our experiment, we applied Daubechies wavelets [26] for filtering.

Also, we considered four other different schemes for the selection of the signal's region of interest; namely, Central Peak positioning (CP), Main Energy capturing (ME) Random Positioning (RA) and Systematical echo capturing method with the preservation of Original neighboring grass (SO). We verified these four methods as well as our own-developed method SZ as preprocesing techniques for ultrasonic signals in Chapter 5. The experimental results showed that the classifiers trained by DWT data using these four methods showed weaker performance than the DWT-based classifier using SZ. Despite their comparative weakness, we included these weak classifiers as members of multi-classifiers.

We used fully connected feed-forward ANNs with 128 input nodes, two hidden layers with 64 nodes and 16 nodes and an output layer with 2 nodes for classifying the shaft signals into cracks (CR) or mode-converted echoes (MC). ${ }^{2}$ We trained using the back-propagation algorithm in batch mode and the topological order as the update mode of the networks. The learning rate was 0.2 and the Mean Square Error limit was 0.01 for stopping the training process. The epoch limit was 200,000 for those occasional cases where training failed to converge. The input samples were randomly divided up into 5 sets. In turn, we used 4 of these to train the network, and the remaining set to validate the network. This was repeated with all five possible combinations, and furthermore, the process was repeated 5 times to get the diversity of the network's training ability by assigning 5 different initial weights to the network. As the result of this process, we produced 150 ANN models trained by six different feature sets; one FFT feature set and five DWT feature sets which were preprocessed using five different schemes (RA, CP, SO, SZ and ME).

The network topology (128-64-16-2) selected for this experiment is different from the topology (128-64-16-1) selected for other ANN experiments in Chapter 3. We selected the two-

\footnotetext{
${ }^{2}$ For the decision of the class, the weight values of two output nodes were compared and the class were considered as $\mathrm{MC}$ if the first value is bigger than the second one and as $\mathrm{CR}$ vice versa.
} 
output-node topology because here we start with extending to ensembles of classifiers. The experiment presented in this chapter is the first step for exploring and applying combining paradigms for the shaft signal classification, in order to gain some fundamental idea of the difference in the classification results according to the difference in the combining methods (homogeneous and heterogeneous). In this experiment, only decisions (whether MC or CR) of each classifier were combined. However, the factors for combining can be extended by incorporating the final weight value of every output node of the ANN, especially when we apply the stacking method. This is one of our future work extended from this thesis and this is mentioned in Section 8.2.

For SVM classifiers, we employed RBF kernels because they provide nonlinear mapping, require comparatively small numbers of hyper parameters and offer fewer numerical difficulties. RBF requires a penalty parameter $C$ and kernel parameter $\gamma$. We used a grid-searching algorithm [37] where pairs of $C$ and $\gamma$ are tried and the one with the best 10-fold-crossvalidation accuracy was picked. The result of the grid searching was the values $4,16,1,2$, 1 and 8 for $\mathrm{C}$ corresponding to the six feature sets FFT, DWT-CP, DWT-ME, DWT-RA, DWT-SO and DWT-SZ. The respective $g$ values are 1/32, 1/32, 1/32, 1/8, $1 / 8$ and 1/32. Again, we used the 5-fold cross-validation test on six SVM models, which are trained by six feature sets. Thus, we manipulated 30 individual SVM classifiers.

\subsubsection{Combining multiple classifiers}

The purpose of our experiment was to empirically find the combination that is most fruitful. Thus, we investigated the impact on classification performance of combinations of multiple classifiers trained by different feature schemes or different learning paradigms. We explored three combining methods, namely Bayesian Combination (BC) [92, 104], Distribution summation (DS) [1, 18] and Likelihood Combination (LC) [1, 30].

In a Bayesian combination method, weights are established proportional to each individual classifier's past performance that are computed by its posterior probability using Bayes' theorem. In the Distribution summation method, distributions with each individual model are presented as a vector that records how many training data are correctly classified for each class. These vectors of multiple models are combined using vectorial addition for making 
the combined decision. The likelihood combination method is a weighted combination in which the Naive Bayes Algorithm is applied to learn weights for classifiers. The details of these three methods are presented in Appendix $\mathrm{C}$ and the result weights calculated through each method for our experiment are shown in Appendix D.

We combined the outputs of two individual models under two streams. The first stream was to combine FFT models and DWT models trained by the same learning algorithm (ANN or SVM). We produced one FFT (data set) type classifier and five different DWT (data sets) type classifiers. Thus, there were five FFT-DWT combinations. Learning with SVM or with ANN from the feature-combination results in 10 ensembles. These are presented in the first two rows in Table 6.1 and Figure 6.5. The second stream was to combine one SVM model with one ANN model trained by one same feature set (FFT or DWT). Thus, we had 6 ensembles corresponding to the third row in Table 6.1 and Figure 6.5. All the combinations were carried out by three different combining methods (the 3 columns within each major column in Table 6.1 and Figure 6.5). The classification accuracy for each class is also recorded separately from the overall accuracy.

\subsection{Results and Analysis}

In order to investigate if the combined model performs more accurately in classifying input data than a single model does, we compared the decision error rate of the combined model with the decision error rate of each individual participant model. Table 6.1 shows the comparison of the decision error rate between combined models and single classifier models by calculating the difference of both error rates. $c(A, B)$ indicates a combined classifier of two individual classifiers model $A$ and model $B$. The figures in rows (1) to (3) indicate the difference of error rates between the combined model and the first single model participant while those in (4) to (6) are the difference of error rates between the combined model and the second participant. Among these six rows, (1) and (4) are the results for classification performance for overall data. On the other hand, (2) and (5) are result figures for classifying CR while (3) and (6) are for classifying MC. Figure 6.5 graphically presents this comparison result, and displays the amount of improvement in the classification performance in a form of bar charts. The Bars where the combination is an improvement point downwards while 
if a single classifier remains better, the bar points upwards. We also computed a value $\phi_{e}$ which indicates the "fraction of correlated errors" [1] and is also listed in Table 6.1 and Figure 6.5. The value of $\phi_{e}$ is generally used to measure the degree to which the errors made by models of the ensemble are correlated.

Table 6.1: Comparison of the performance of combined models. $c(A, B)$ indicates a combined classifier of two individual classifiers model $A$ and model $B$. The figures presented in (1) to (3) are the differences of error rates between the combined model and the first single model participant, and those in (4) to (6) are corresponding figures between the combined model and the second participant. Among the six rows, (1) and (4) are the results for overall, (2) and (5) are for classifying CR and (3) and (6) are for classifying MC

\begin{tabular}{|c|c|c|c|c|c|c|c|c|c|c|c|c|c|c|c|c|c|c|}
\hline Learning algorithm & \multicolumn{15}{|c|}{ SVM } & & & \\
\hline Ensemble & \multirow{2}{*}{\multicolumn{3}{|c|}{$\frac{\text { C(FFT,DWT-CP) }}{3.75}$}} & \multicolumn{3}{|c|}{$C$ (FFT,DWT-ME) } & \multicolumn{3}{|c|}{ C(FFT,DWT-RA) } & \multirow{2}{*}{\multicolumn{3}{|c|}{$\frac{C(F F T, D W T-S O)}{7.5}$}} & \multicolumn{3}{|c|}{ C(FFT,DWT-SZ) } & & & \\
\hline$\phi_{e}$ & & & & \multicolumn{3}{|c|}{11.33} & \multicolumn{3}{|c|}{12.58} & & & & \multicolumn{3}{|c|}{5} & & & \\
\hline Combining method & DS & \begin{tabular}{l|l}
$\mathrm{BC}$ \\
\end{tabular} & LC & DS & $\mathrm{BC}$ & \begin{tabular}{|l|}
$\mathrm{LC}$ \\
\end{tabular} & DS & $\mathrm{BC}$ & LC & DS & $\mathrm{BC}$ & LC & $\mathrm{DS}$ & $\mathrm{BC}$ & LC & & & \\
\hline (1) & 0.006 & 0.006 & 0.006 & -0.04 & -0.018 & -0.018 & 0.010 & 0.01 & 0.033 & -0.069 & -0.047 & -0.047 & -0.04 & -0.04 & -0.04 & & & \\
\hline (2) & -0.095 & -0.175 & -0.175 & 0.018 & -0.119 & -0.119 & 0.058 & 0.035 & -0.051 & 0.007 & -0.182 & -0.182 & -0.022 & -0.13 & -0.13 & & & \\
\hline (3) & -0.05 & -0.077 & -0.077 & -0.037 & -0.088 & -0.063 & 0.014 & 0.013 & -0.013 & -0.048 & \begin{tabular}{l|l|}
-0.102 \\
\end{tabular} & -0.102 & -0.048 & -0.088 & -0.088 & & & \\
\hline (4) & -0.103 & -0.103 & -0.103 & -0.044 & -0.022 & -0.022 & -0.119 & -0.119 & -0.096 & 0 & 0.022 & 0.022 & -0.093 & -0.093 & -0.093 & & & \\
\hline (5) & 0.206 & 0.126 & 0.126 & 0.075 & -0.062 & -0.062 & -0.017 & -0.04 & -0.126 & 0.08 & -0.109 & -0.109 & 0.109 & 0 & & & & \\
\hline (6) & 0.014 & -0.013 & -0.013 & -0.012 & -0.063 & -0.038 & -0.077 & -0.078 & -0.103 & 0.027 & -0.027 & -0.027 & -0.024 & -0.063 & -0.063 & & & \\
\hline Learning algorithm & \multicolumn{15}{|c|}{ ANN } & & & \\
\hline Ensemble & \multirow{2}{*}{\multicolumn{3}{|c|}{$\frac{C(\text { FFT,DWT-CP) }}{3.78}$}} & \multicolumn{3}{|c|}{ C(FFT,DWT-ME) } & \multicolumn{3}{|c|}{$C$ (FFT,DWT-RA) } & \multirow{2}{*}{\multicolumn{3}{|c|}{$\frac{C \text { (FFT,DWT-SO) }}{7.27}$}} & \multicolumn{3}{|c|}{ C(FFT,DWT-SZ) } & & & \\
\hline$\phi_{e}$ & & & & \multicolumn{3}{|c|}{3.78} & \multicolumn{3}{|c|}{12.65} & & & & \multicolumn{3}{|c|}{6.03} & & & \\
\hline Combining method & DS & $\mathrm{BC}$ & LC & DS & $\mathrm{BC}$ & \begin{tabular}{|l|}
$\mathrm{LC}$ \\
\end{tabular} & DS & $\mathrm{BC}$ & LC & DS & $\mathrm{BC}$ & LC & DS & $\mathrm{BC}$ & LC & & & \\
\hline (1) & -0.104 & -0.178 & -0.163 & -0.11 & -0.178 & -0.174 & -0.093 & -0.091 & -0.087 & -0.111 & \begin{tabular}{|l|}
-0.128 \\
\end{tabular} & -0.124 & -0.071 & -0.135 & -0.125 & & & \\
\hline (2) & 0.045 & 0 & 0.004 & 0.032 & -0.013 & -0.01 & 0.128 & 0.107 & 0.107 & 0.109 & 0.023 & 0.031 & 0.125 & 0.024 & 0.016 & & & \\
\hline (3) & -0.064 & -0.106 & -0.096 & -0.072 & -0.111 & -0.106 & -0.01 & -0.013 & -0.01 & -0.034 & -0.073 & -0.067 & -0.015 & -0.069 & -0.067 & & & \\
\hline (4) & -0.021 & -0.095 & -0.079 & -0.043 & -0.111 & -0.107 & -0.225 & -0.223 & -0.219 & -0.202 & -0.219 & -0.215 & -0.082 & -0.146 & -0.136 & & & \\
\hline (5) & 0.127 & 0.082 & 0.085 & 0.132 & 0.087 & 0.09 & -0.069 & -0.09 & -0.09 & 0.08 & -0.006 & 0.002 & 0.04 & -0.061 & -0.069 & & & \\
\hline (6) & 0.016 & -0.026 & -0.016 & 0.013 & -0.026 & -0.021 & -0.172 & -0.175 & -0.173 & -0.075 & -0.113 & -0.108 & -0.057 & -0.112 & -0.109 & & & \\
\hline Learning algorithm & \multicolumn{3}{|c|}{ FFT } & \multicolumn{3}{|c|}{ DWT-CP } & \multicolumn{3}{|c|}{ DWT-ME } & & DWT-RA & & & DWT-SZ & & & DWT-SO & \\
\hline Ensemble & $C(\mathrm{~S}$ & VM,AN & & $C(\mathrm{~S}$ & $\mathrm{VM}, \mathrm{AN}$ & & $C(\mathrm{~S}$ & $\mathrm{VM}, \mathrm{AN}$ & & $C(\mathrm{~S}$ & VM,AN & & & $\mathrm{VM}, \mathrm{AN}$ & & $C(\mathrm{~S}$ & VM,AN & \\
\hline$\phi_{e}$ & & 13.13 & & & 9.25 & & & 4.32 & & & 22.63 & & & 9.17 & & & 14 & \\
\hline Combining method & DS & $\mathrm{BC}$ & LC & DS & $\mathrm{BC}$ & LC & DS & $\mathrm{BC}$ & LC & DS & $\mathrm{BC}$ & LC & $\mathrm{DS}$ & $\mathrm{BC}$ & LC & DS & $\mathrm{BC}$ & LC \\
\hline (1) & 0.017 & 0.039 & 0.039 & -0.064 & -0.064 & -0.064 & -0.01 & 0.019 & 0.019 & -0.029 & 0.009 & 0.009 & 0.009 & 0.013 & 0.013 & -0.015 & -0.01 & -0.01 \\
\hline (2) & -0.056 & -0.095 & -0.095 & 0.093 & 0.048 & 0.048 & -0.091 & -0.118 & -0.118 & 0.02 & 0.001 & 0 & -0.014 & -0.037 & -0.012 & 0.043 & 0.015 & 0.015 \\
\hline (3) & -0.022 & -0.025 & -0.025 & -0.002 & -0.015 & -0.015 & -0.058 & -0.052 & -0.052 & 0.002 & 0.009 & 0.009 & 0 & -0.008 & 0.003 & 0.005 & -0.005 & -0.005 \\
\hline (4) & -0.137 & -0.115 & -0.115 & -0.025 & -0.025 & -0.025 & -0.093 & -0.065 & -0.065 & -0.187 & -0.149 & -0.149 & -0.305 & -0.301 & -0.301 & -0.128 & -0.124 & -0.124 \\
\hline (5) & 0.056 & 0.017 & 0.017 & -0.015 & -0.059 & -0.059 & 0.064 & 0.036 & 0.036 & \begin{tabular}{|l|}
0.01 \\
\end{tabular} & -0.01 & -0.01 & -0.004 & -0.026 & -0.001 & -0.059 & \begin{tabular}{|l|}
-0.088 \\
\end{tabular} & -0.088 \\
\hline (6) & -0.06 & -0.063 & -0.063 & -0.023 & -0.037 & -0.037 & -0.038 & -0.032 & -0.032 & -0.107 & -0.099 & -0.099 & -0.154 & -0.161 & -0.151 & \begin{tabular}{|l|}
-0.1 \\
\end{tabular} & -0.11 & -0.11 \\
\hline
\end{tabular}

The following points are noteworthy from the experimental result.

- Combined models show better performance than single models in the classification accuracy for the whole test set across schema for generating or combining multiclassifiers. Note that most of the histograms in Figure 6.5 show the superiority of the combining models over the single models.

- Combining two classifiers trained by different feature sets becomes more advantageous when we use SVM as a learning algorithm than when we use ANN. Note that most of the histograms in Figure 6.5 show the superiority of the combining models over the 

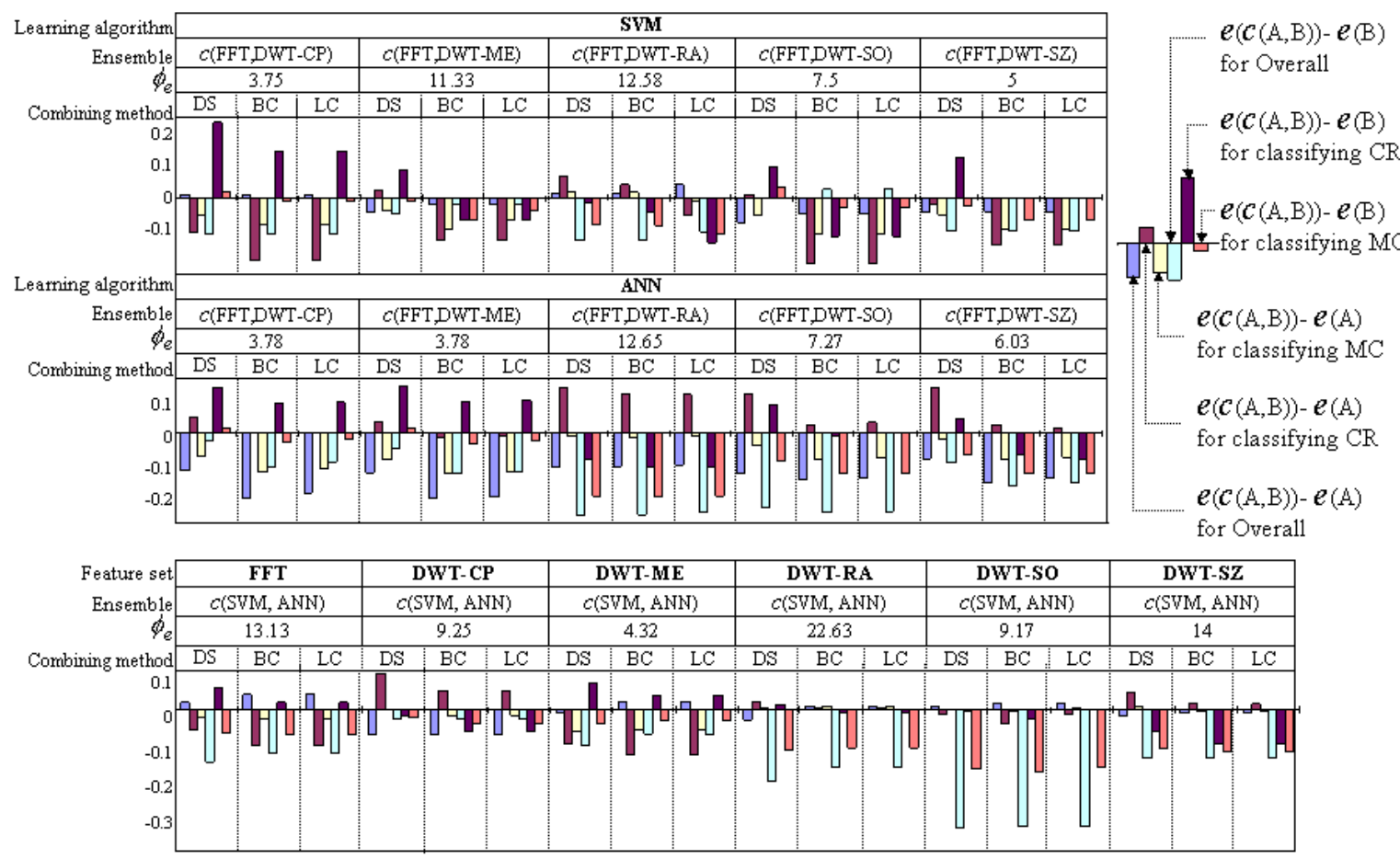

Figure 6.5: Bar charts for the comparison of the performance of combined models. $c(A, B)$ indicates a combined classifier of two individual classifiers, model $A$ and model $B$. $e(A)$ indicates the classification error rate of the classifier model $A$. 
single models.

- Though the overall accuracy of combined models is higher than the accuracy of single models across most types of combinations, their performance in classifying each class data (MO and CR) is diverse. Especially, most FFT and DWT ensembles trained by ANN perform worse than single models in classifying CR data; whilst corresponding combined models trained by SVM perform reliably on both classes except for one combination (the FFT and DWT-CP ensemble).

- Amongst the five types of DWT data combined with FFT data, DWT-SZ shows most reliability in classifying both classes regardless of the learning paradigm. This implies that different echo gating preprocessing for extracting DWT features plays a role in structuring the DWT feature sets. We suspect there are some implicit differences in DWT.

- The performance of the heterogeneously combined classifiers is different depending on which feature sets were used to train them.

- The value of $\phi_{e}$ is related to the amount of error reduction made by combining multiclassifiers. As shown in Figure 6.5, the value of $\phi_{e}$ seems to be very relevant to the overall error reduction rate. It seems not to have much relevance to the error reduction for each class data.

- The most suitable combination structure may depend on the interest of some particular class. For example, if accuracy for the CR class is the issue, then the SVM with DWT (single classifier) is not surpassed by the combination, although the combination does better over all the classes.

\subsection{Remarks}

We have explored the combining of classifiers along the dimension of a feature extraction mechanism, along the dimension of a combination method and along the dimension of the type of classifier. This experimental result confirms three points for designing an integrated multi-classifier system for shaft test data by way of selectively employing the combining 
structure used in this experiment. First, combination in general improves the accuracy, and second, combining features has the potential for improvement. Third, in particular, the most productive combination that offers the most improvement is usually a combination of ANN and SVM trained by DWT-based feature vectors. 


\section{Chapter 7}

\section{A Cost-Sensitive Integrated SVM Classification System for Ultrasonic Signal Tests}

\subsection{Introduction}

In Chapter 6, we explored the diverse possibilities of heterogeneous and homogeneous ensembles, combination techniques, feature extraction methods and classifier types and provided guidelines about which combinations result in superior performance. The analysis of the experimental results obtained in Chapter 6 implies that combination in general improves the accuracy, and combining features has the potential for improvement in discriminating ultrasonic shaft test signals. The experimental results, therefore, become the basis for the construction of an integrated multi-classifier model using both of the feature extraction schemes (FFT and DWT) effectively.

As mentioned in previous chapters of this thesis, the main interest for the AUSC research has been the extraction of the most effective set of features from which classification might be performed more efficiently and accurately. While it is hard to determine which set of features is best, it is important to at least identify those that make the process reliable and 
effective in the field. In particular, two feature extraction approaches (FFT and DWT) have been explored as two main candidates to identify ultrasonic signal features for shaft tests. We compared those two feature extraction schemes by using them in classifying ultrasonic shaft signal echoes. For the comparison analysis of those two feature extraction schemes, we employed two supervised learning algorithms (ANN and SVM) to construct classifiers trained by FFT-based feature sets or DWT-based feature sets. The results established experimentally that DWT, when compared to FFT, was a potentially stronger feature extraction scheme for both the ANN-based classifiers and the SVM-based classifiers for classifying echoes from ultrasonic signals in long shafts. It also showed that the DWT scheme enhanced training data more reliably, so it could be selected with higher flexibility, and then it could be used for in-field ultrasonic shaft signal classifiers.

If we insist on a single classifier system only, we have to construct a classifier by employing only DWT as its feature extraction scheme, based on the comparison result which established the superiority of the DWT over the FFT. While this may improve the overall accuracy of the classifier, it will potentially ignore important features that the FFT is highlighting. Also, even if a classifier using one feature extraction scheme seems superior in terms of accuracy rate, we suspect adoption of the best single classifier results in some benefits even in an error-cost-sensitive environment.

Another idea for building a single SVM model is to use all of the available inputs (FFT+DWT) which is a concatenation of FFT-based features and DWT-based features. Therefore, before designing a combined SVM classifier, we made a pre-test of SVM using all available feature inputs (FFT+DWT). We constructed an SVM using a longer vector (FFT+DWT) and compared its accuracy with a single SVM trained by FFT or DWT individually (lets say FSVM or DSVM). As shown in Table 7.1, this pre-test resulted that the performance of the SVM using FFT+DWT was not better than the performance of the superior individual SVM (it was DSVM). Motivated by this result, we plan to seek for a combined system in a different way and the resulted product is an integrated SVM classifier (ISVM) which will be introduced through this chapter.

In this chapter, we show how we have combined classifiers, considering the misclassification cost, to obtain a more informative classification for its application in the field. In order to combine multiple classifiers for this purpose, among various methods of generat- 
Table 7.1: The classification accuracy of SVMs using different input vectors: FFT vs DWT vs $(\mathrm{FFT}+\mathrm{DWT})$

\begin{tabular}{|c|c|c|c|c|}
\cline { 3 - 4 } \multicolumn{2}{c|}{} & \multicolumn{3}{c|}{ Class Accuracy (\%) } \\
\cline { 3 - 5 } \multicolumn{2}{c|}{} & CR & MC & Overall \\
\hline \multirow{2}{*}{$\begin{array}{c}\text { Feature } \\
\text { vectors } \\
\text { for } \\
\text { SVM }\end{array}$} & FFT & 67.2 & 92.5 & 79 \\
\cline { 2 - 5 } & DWT & 93.1 & 83.5 & 86.7 \\
\cline { 2 - 5 } & FFT+DWT & 87.3 & 85.0 & 85.4 \\
\hline
\end{tabular}

ing multiple-classifiers for ensembles described in Section 6.2, we employ a homogeneous approach using multiple feature extraction schemes (FFT and DWT) and a single learning algorithm (SVM). According to the experimental analysis presented in Section 6.4, combining two classifiers trained by different feature sets becomes more advantageous when we use SVM as a learning algorithm than when we use ANN. Based on this result, we employ SVM as the single learning algorithm for the integrated system presented in this chapter.

Our presentation continues in Section 7.2 with a description of our analysis based on costsensitive environments for developing a new integrated SVM classifier (ISVM). In this section, we describe how a new combined decision rule is made to construct the ISVM. Section 7.3 describes our empirical evaluation of the ISVM by comparing its classification performance to a single classifier system using one feature extraction scheme, either FFT or DWT. This is followed by final remarks from these experiments in Section 7.4.

\subsection{A Cost-Sensitive Integrated Classification System}

Our goal is to develop a new integrated SVM classifier (ISVM), that is a combined classification system efficiently employing the strengths of each of two SVM classifiers using two different feature extraction schemes FFT and DWT. To achieve this, firstly two independent SVM classifiers are constructed, namely FSVM and DSVM. FSVM is a SVM classifier trained with the training data whose features are extracted by the FFT scheme while DSVM is trained using data transformed by the DWT. With these two sub-classifiers, the ISVM works by defining new classification rules by which one among FSVM and DSVM 
is chosen and applied to the classification of new data. That is, the new rules determine which sub-classifier's decision has to be observed for one input to ISVM, mainly based on the comparison of posterior probabilities on each case. The overall structure of ISVM is shown in Figure 7.1.

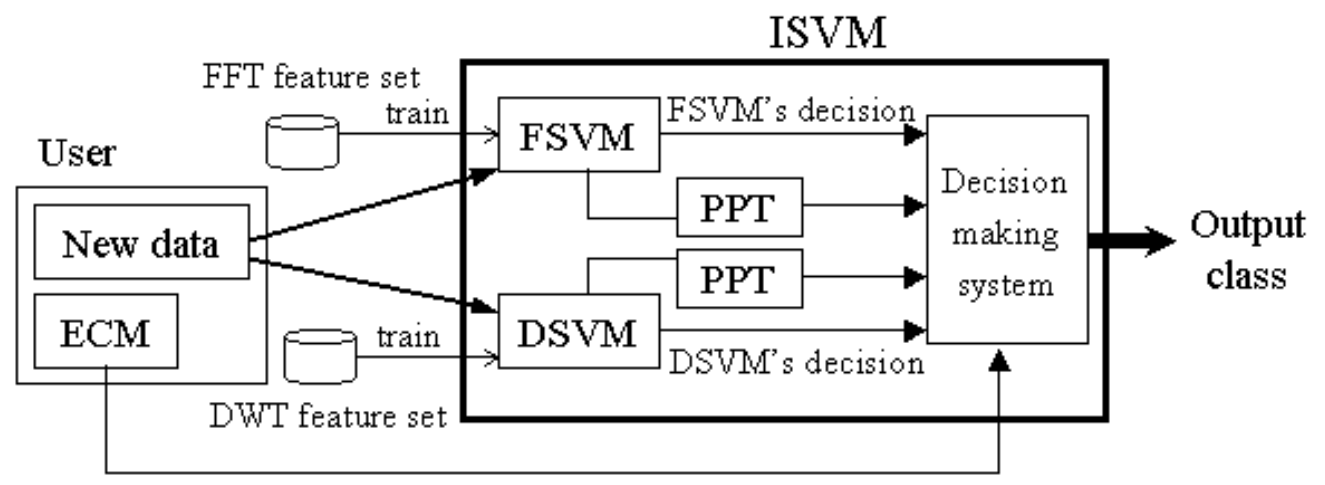

Figure 7.1: The overall structure of ISVM: A new combined decision making system of ISVM is constructed using three components: 1. Individual decisions from FSVM and DSVM. 2. Posterior Probability Table (PPT) calculated on each decision made by FSVM and DSVM. 3. Error-Cost Matrix (ECM) predefined for each type of misclassification.

\subsubsection{Posterior Probability Table and Confusion Matrix Table}

The first step for constructing a new combined decision rule is to calculate the posterior probability of every possible hypothesis on each decision made by FSVM or DSVM. The posterior probability is calculated using Bayes theorem and summarized on a posterior probability table (PPT). In fact, the calculation process using Bayes theorem can be compactly replaced by the representation of the confusion matrix table (CMT) as shown in Table 7.2.

Generally, the CMT shows how well each classifier can recognize each class (sensitivity). For example in Table 7.2, we can see how well FSVM performs when classifying MC samples by simply calculating the sensitivity of MC samples in FSVM (this is $\mathrm{N}_{F M M} / \mathrm{N}_{M C}$ ). Also, we can see the performance of FSVM in classifying CR data is $\mathrm{N}_{F C C} / \mathrm{N}_{C R}$, which is the sensitivity of CR samples in FSVM.

On the other hand, the posterior probability on PPT reflects the confidence that each class 
Table 7.2: The relation between CMT (a) and PPT (b) and examples of CMT (c) and $\operatorname{PPT}(\mathrm{d})$

(a) CMT

\begin{tabular}{|c|c|c|c|c|c|}
\hline \multirow{2}{*}{$\begin{array}{c}\text { Real } \\
\text { Class }\end{array}$} & \multirow{2}{*}{ Qty } & \multicolumn{2}{|c|}{$\begin{array}{c}\text { FSVM's } \\
\text { decision }\end{array}$} & \multicolumn{2}{c|}{$\begin{array}{c}\text { DSVM's } \\
\text { decision }\end{array}$} \\
\cline { 3 - 6 } & & MC & CR & MC & CR \\
\hline MC & \multirow{2}{*}{ NMC $_{\text {N }}$} & $N_{F M M}$ & $N_{F M C}$ & $N_{D M M}$ & $N_{D M C}$ \\
\hline CR & $N_{C R}$ & $N_{F C M}$ & $N_{F C C}$ & $N_{D C M}$ & $N_{D C C}$ \\
\hline
\end{tabular}

(D: training data $\mathrm{H}$ : hypothesis) (b) PPT

\begin{tabular}{|c|c|c|c|}
\hline & \multicolumn{2}{|c|}{ Real Class } \\
\hline & & $\mathrm{MC}$ & CR \\
\hline \multirow{2}{*}{$\begin{array}{l}\text { FSVM's } \\
\text { decision }\end{array}$} & $\mathrm{MC}$ & $\begin{array}{c}N_{F M G M} /\left(N_{F M G M}+N_{F C M}\right) \\
(t f m)\end{array}$ & $\begin{array}{c}N_{F C M} /\left(N_{F M a n}+N_{F C M}\right) \\
(e f m)\end{array}$ \\
\hline & CR & $\begin{array}{c}N_{F M C} /\left(N_{F M C}+N_{F C C}\right) \\
(e f c)\end{array}$ & $\begin{array}{c}\mathrm{N}_{F C C}\left(\mathrm{~N}_{\mathrm{FMC}}+\mathrm{N}_{\mathrm{FC} C}\right) \\
(\mathrm{tfc})\end{array}$ \\
\hline \multirow{2}{*}{$\begin{array}{l}\text { DSVM's } \\
\text { decision }\end{array}$} & $\mathrm{MC}$ & $\begin{array}{c}N_{D M M N}\left(N_{D M a n}+N_{D C M}\right) \\
(\operatorname{tdm})\end{array}$ & $\begin{array}{c}N_{D M M}\left(\mathrm{~N}_{D M M M}+\mathrm{N}_{D C M}\right) \\
(\mathrm{edm})\end{array}$ \\
\hline & CR & $\begin{array}{c}\mathrm{NDMC}_{\mathrm{DM}}\left(\mathrm{N}_{\mathrm{DMC} C}+\mathrm{N}_{\mathrm{DCC}}\right) \\
(\mathrm{edc})\end{array}$ & $\begin{array}{c}\mathrm{N}_{D C C} /\left(\mathrm{N}_{\mathrm{DMC} C}+\mathrm{N}_{\mathrm{DCC}}\right) \\
(\mathrm{tdc})\end{array}$ \\
\hline
\end{tabular}

$\mathrm{P}(\mathrm{H} \mid \mathrm{D})$

(d) An example of PPT

(c) An example of CMT

\begin{tabular}{|c|c|c|c|c|c|}
\hline \multirow{2}{*}{$\begin{array}{c}\text { Real } \\
\text { Class }\end{array}$} & \multirow{2}{*}{ Qty } & \multicolumn{2}{|c|}{$\begin{array}{c}\text { FSVM's } \\
\text { decision }\end{array}$} & \multicolumn{2}{c|}{$\begin{array}{c}\text { DSVM's } \\
\text { decision }\end{array}$} \\
\cline { 3 - 6 } & & MC & CR & MC & CR \\
\hline MC & 69 & 65 & 4 & 57 & 12 \\
\hline CR & 50 & 18 & 32 & 3 & 47 \\
\hline
\end{tabular}


holds after we have made one decision by a classifier. For example, the value $t f m$ in the PPT table shown on Table 7.2(b) reflects how much we can be confident that the real class of an input data would be MC when FSVM classifies an input data as MC. Correspondingly, the value efm is the probability that the decision in FSVM, which is MC, would be wrong. Therefore, the value $\mathrm{tfm}$ is also considered as the degree of trust about the FSVM's decision on $\mathrm{MC}$ while the value efm is the error rate on that decision.

Therefore, the CMT in Table 7.2(a) summarizes test results through two already constructed classifiers, FSVM and DSVM. Based on the CMT, the trust-rates and error-rates are calculated on each decision. The completed PPT becomes the basis of the further decision which will be made on ISVM. The process for the classification of a new instance in ISVM is divided into two steps as follows.

1. The input data is classified by the sub-classifiers (FSVM and DSVM), then the results are recorded as $\mathrm{F}$ and $\mathrm{D}$.

2. Two posterior probabilities $\mathrm{P}(\mathrm{CR} \mid \mathrm{F}, \mathrm{D})$ and $\mathrm{P}(\mathrm{MC} \mid \mathrm{F}, \mathrm{D})$ are compared. CR is assigned to the new instance if $\mathrm{P}(\mathrm{CR} \mid \mathrm{F}, \mathrm{D})$ is higher than $\mathrm{P}(\mathrm{MC} \mid \mathrm{F}, \mathrm{D})$, and $\mathrm{MC}$ is assigned in the opposite case. Here, values on PPT of each sub-classifiers are used to calculate the posterior probabilities for the combined $\operatorname{cases}(\mathrm{P}(\mathrm{CR} \mid \mathrm{F}, \mathrm{D})$ or $\mathrm{P}(\mathrm{MC} \mid \mathrm{F}, \mathrm{D}))$.

This decision rule partly employs the theory of naive Bayes classifier and is more or less straightforward if the cost is assigned evenly to different types of misclassification. For example, the final decision made by ISVM is always to follow DSVM's result when we use the example PPT shown in Table 7.2(d). In this case, ISVM is of less interest because the integrated system will never use the potential of FSVM. However, in a cost-sensitive environment where one needs to assign different costs to different types of classification error, the decision making rule in ISVM becomes more complex. We discuss further the cost-sensitiveness for different types of error in AUSC systems.

\subsubsection{Cost of Misclassification Errors}

In conventional or simple classification systems, the cost for making errors in classification is usually the same regardless of the type of misclassification. It is, however, impractical 
to ignore the difference in costs for the different types of errors. In such a cost-sensitive environment, the classification system seeks to minimize the total misclassification cost, while the conventional classification seeks to minimize the total number of errors regardless of cost.

In the field of shaft inspection data where we need to predict whether a signal echo is caused by $\mathrm{CR}$ (crack) or MC(mode-conversion), two different costs can be assigned. One is the cost of having a crack undetected (assigning the input to class $\mathrm{MC}$ when it actually belongs in class CR), and the other is the cost of having a false alarm (assigning the input to class CR when it actually belongs in class $\mathrm{MC})$. These costs are denoted as $c(\mathrm{MC}, \mathrm{CR})$ and $c(\mathrm{CR}, \mathrm{MC})$ respectively, as shown in the Error Cost Matrix (ECM) in Table 7.3(a). Here we assume the cost is zero when a case is classified accurately and we exclude the cost of assigning a case to the unknown class. This ECM becomes a basic component for constructing the decision making rule in ISVM, which consequently becomes a cost-sensitive classification system.

Table 7.3: Error-cost for each type of misclassification (a) and the calculation formulas of expected risk for each case (b)

(a) Error-cost matrix (ECM)

\begin{tabular}{|c|c|c|}
\hline \multirow{2}{*}{$\begin{array}{c}\text { Real } \\
\text { Class }\end{array}$} & \multicolumn{2}{|c|}{ Classifier's decision } \\
\cline { 2 - 3 } $\mathrm{MC}$ & $c(\mathrm{MC}, \mathrm{MC})=0$ & $c(\mathrm{CR}, \mathrm{MC})=b$ \\
\hline $\mathrm{MC}$ & $c(\mathrm{MC}, \mathrm{CR})=a$ & $c(\mathrm{CR}, \mathrm{CR})=0$ \\
\hline
\end{tabular}

(b) Expected risk for each decision

\begin{tabular}{|c|c|c|}
\hline \multicolumn{2}{|c|}{$\begin{array}{c}\text { Classifier's } \\
\text { Decision }\end{array}$} & Expected Risk (ER) \\
\hline \multirow{2}{*}{ FSVM } & MC & $\begin{array}{c}c(\mathrm{MC}, \mathrm{CR}) \mathrm{P}(\mathrm{CR} \mid \mathrm{FSVM}-\mathrm{MC}) \\
=a^{*} \mathrm{efm}\end{array}$ \\
\cline { 2 - 3 } & $\mathrm{CR}$ & $\begin{array}{c}c(\mathrm{CR}, \mathrm{MC}) \mathrm{P}(\mathrm{MC} \mid \mathrm{FSVM}-\mathrm{CR}) \\
=b^{*} \mathrm{efc}\end{array}$ \\
\hline DSVM & $\mathrm{MC}$ & $\begin{array}{c}c(\mathrm{MC}, \mathrm{CR}) \mathrm{P}(\mathrm{CR} \mid \mathrm{FSVM}-\mathrm{MC}) \\
=a^{*} \mathrm{edm}\end{array}$ \\
\cline { 2 - 3 } & $\mathrm{CR}$ & $\begin{array}{c}c(\mathrm{CR}, \mathrm{MC}) \mathrm{P}(\mathrm{MC} \mid \mathrm{FSVM}-\mathrm{CR}) \\
=b^{*} \mathrm{edc}\end{array}$ \\
\hline
\end{tabular}

To minimize the expected value of the classification (named expected risk) in a typical errorsensitive classification system, the optimal decision rule is to assign an input pattern $\mathbf{x}$ to the class $k$ such that:

$$
k=\arg \min _{i=1, \ldots, m} \sum_{j=1}^{m} c(i, j) P(j \mid \mathbf{x})
$$

where $c(i, j)$ denotes the cost of deciding for class $i$ when the true class is $j, m$ is the number 
of classes, and $P(j \mid \mathbf{x})$ denotes the $j$ th class posterior probability for pattern $\mathbf{x}$.

When we apply this rule to the classification of shaft inspection data, the Equation(7.1) is transformed to:

$$
k=\arg \min _{i \in\{C R, M C\}}(c(i, C R) P(C R \mid x)+c(i, M C) P(M C \mid x))
$$

Therefore, the expected risk for every misclassification case in the sub-classifiers FSVM and DSVM is summarized in Table 7.3(b) and these values are supplied to the decision making rule in ISVM.

\subsubsection{Decision-Making Rule in ISVM}

As shown in Figure 7.1, ISVM is an integrated system which consists of two sub-classifiers FSVM and DSVM. A different decision rule is applied depending on the types of combination of two sub-decision results from FSVM and DSVM. For one specific piece of shaft inspection data as an input to ISVM, we have four cases in the combination of two decisions by FSVM and DSVM and they are presented in Table 7.4. The expected risk (ER) for each of four combinations is also formulated using values calculated through ECM (Table 7.3(a)) and PPT (Table 7.2(b)). The calculated ERs for all four hypotheses are compared and the one with the smallest ER is chosen as the final decision in ISVM.

Table 7.4: Expected Risk (ER) for each hypothesis on ISVM's decision and derived rules for final decision

\begin{tabular}{|c|c|c|c|c|}
\hline \multicolumn{2}{|c|}{$\begin{array}{l}\text { Sub-classifier's } \\
\text { decision }\end{array}$} & \multicolumn{2}{|c|}{$\begin{array}{l}\text { ER for ISVM's } \\
\text { decision }\end{array}$} & \multirow{2}{*}{$\begin{array}{c}\text { Final decision rule } \\
\text { (assume } \mathrm{r}=c(\mathrm{MC}, \mathrm{CR}) / c(\mathrm{CR}, \mathrm{MC})=\mathrm{a} / \mathrm{b})\end{array}$} \\
\hline FSVM & DSVM & MC & CR & \\
\hline $\mathrm{MC}$ & $\mathrm{MC}$ & $a^{*} \mathrm{efm}^{*} \mathrm{edm}$ & $b^{*} \mathrm{tfm}{ }^{*} \mathrm{tdm}$ & $\mathrm{MC}$ if $r<r_{4}$ and CR if $r>r_{4}$, where $r_{4}=\mathrm{tfm} * \mathrm{tdm} / \mathrm{efm} * \mathrm{edm}$ \\
\hline $\mathrm{CR}$ & $\mathrm{CR}$ & $a^{*}+f_{c}^{* *} t d c$ & $b^{*}$ efc ${ }^{*} \mathrm{edc}$ & $\mathrm{MC}$ if $r<r_{l}$ and CR if $r>r_{l}$, where $r_{l}=\mathrm{efc}^{*} \mathrm{edc} / \mathrm{tfc} * \mathrm{tdc}$ \\
\hline $\mathrm{MC}$ & $\mathrm{CR}$ & $a^{*}$ efm ${ }^{*} t d c$ & $b^{*} \mathrm{tfm} * \mathrm{edc}$ & $\mathrm{MC}$ if $r<r_{2}$ and CR if $r>r_{2}$, where $r_{2}=\mathrm{tfm} * \mathrm{edc} / \mathrm{efm} * \mathrm{tdc}$ \\
\hline $\mathrm{CR}$ & $\mathrm{MC}$ & $a^{*} \mathrm{tfc}^{*} \mathrm{edm}$ & $b^{*}$ efc $*$ tdm & $\mathrm{MC}$ if $r<r_{3}$ and $\mathrm{CR}$ if $r>r_{3}$, where $r_{3}=\mathrm{efc} * \mathrm{tdm} / \mathrm{tfc}{ }^{*}$ edm \\
\hline
\end{tabular}

We can also present the decision rule in a different way, which makes it more explanatory.

- If the decisions of FSVM and DSVM are equal and $r$ is smaller than $r_{1}$, assign MC. 
- If the decisions of FSVM and DSVM are equal and $r$ is larger than $r_{4}$, assign CR.

- If the decisions of FSVM and DSVM are equal and $r$ is between $r_{1}$ and $r_{4}$, follow their decision.

- If the decisions of FSVM and DSVM are different and $r$ is between $r_{2}$ and $r_{3}$, follow the opinion of DSVM.

- If the decisions of FSVM and DSVM are different and $r$ is smaller than $r_{2}$, assign MC.

- If the decisions of FSVM and DSVM are different and $r$ is larger than $r_{3}$, assign CR.

The whole procedure of making the decision rule in ISVM is summarized in Figure 7.2 as a flow chart.

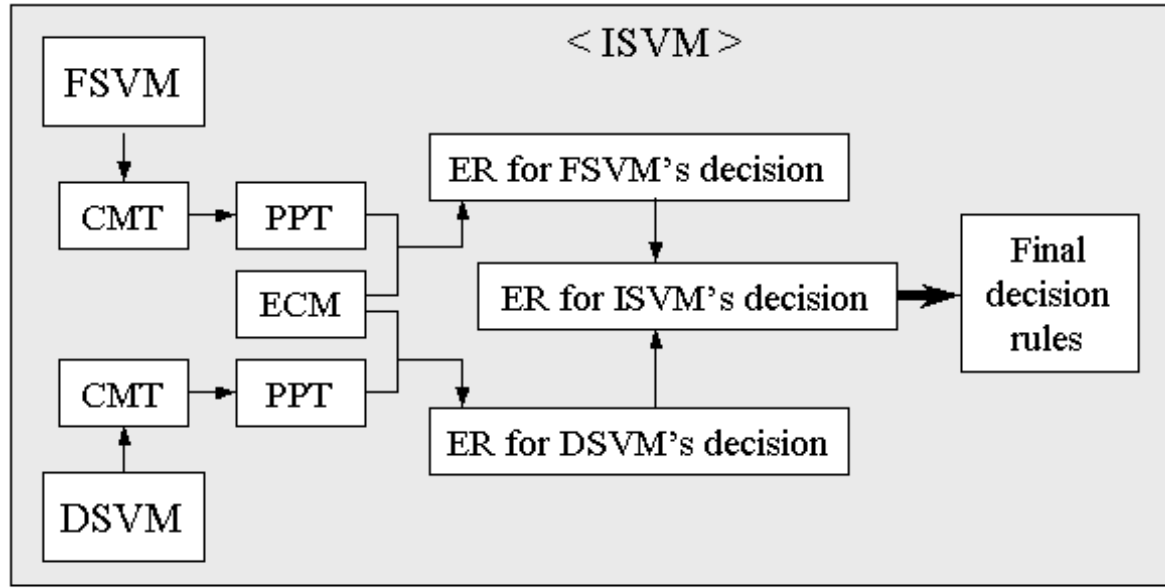

- FSVM : FFT-based SVM classifier

- CMT : Confusion Matrix Table

- ECM : Error-Cost Matrix
- DSVM : DWT-based SVM classifier

- PPT : Posterior Probability Table

- ER : Expected Risk

Figure 7.2: Flow chart of overall procedure of constructing the decision rule in ISVM. 


\subsection{Empirical Evaluation}

We set up an experiment for evaluating the newly developed ISVM. The experiment consists of four main processes.

1. We preprocess a set of shaft inspection data, which will be used as a training and validation set, and mapped to feature domains using two feature extraction schemes (FFT and DWT).

2. We train two SVM models (temp_FSVM and temp_DSVM) with FFT-based training data and DWT-based training data respectively. We obtain the test result through a 10-fold cross validation. Based on this test result, a PPT is constructed for each model.

3. Two SVM models (FSVM and DSVM) are trained with the whole set of FFT training data and the whole set of DWT training data respectively. These two trained SVM models become sub-classifiers in ISVM.

4. We prepare a separate validation set for each domain and classify them with FSVM and DSVM. These classification results are compared with the result classified by the new developed decision rule of ISVM.

Figure 7.3 summarizes the steps of our experiment. A more detailed description follows.

The procedure for the construction of the database for the experiment is similar to the one we followed in other experiments in previous chapters of this thesis. That is, we acquired A-scan signals from eight various shafts, ranging from $100 \mathrm{~mm}$ to $1300 \mathrm{~mm}$ long by using a probe set to $2 \mathrm{MHz}$ frequency. Signal segments of interest then were selected from the whole ultrasonic A-scan signals. In order to apply a consistent method of signal segmentation which is necessary for suppressing time-variance problems with DWT, we used a systematic echo capturing method with Zero-padding (SZ) described in Chapter 5.

Through this process, we produced a set of time-domain signal vectors of 768 samples long. We also recorded whether the signal originated from a crack (CR) or not and whether it 


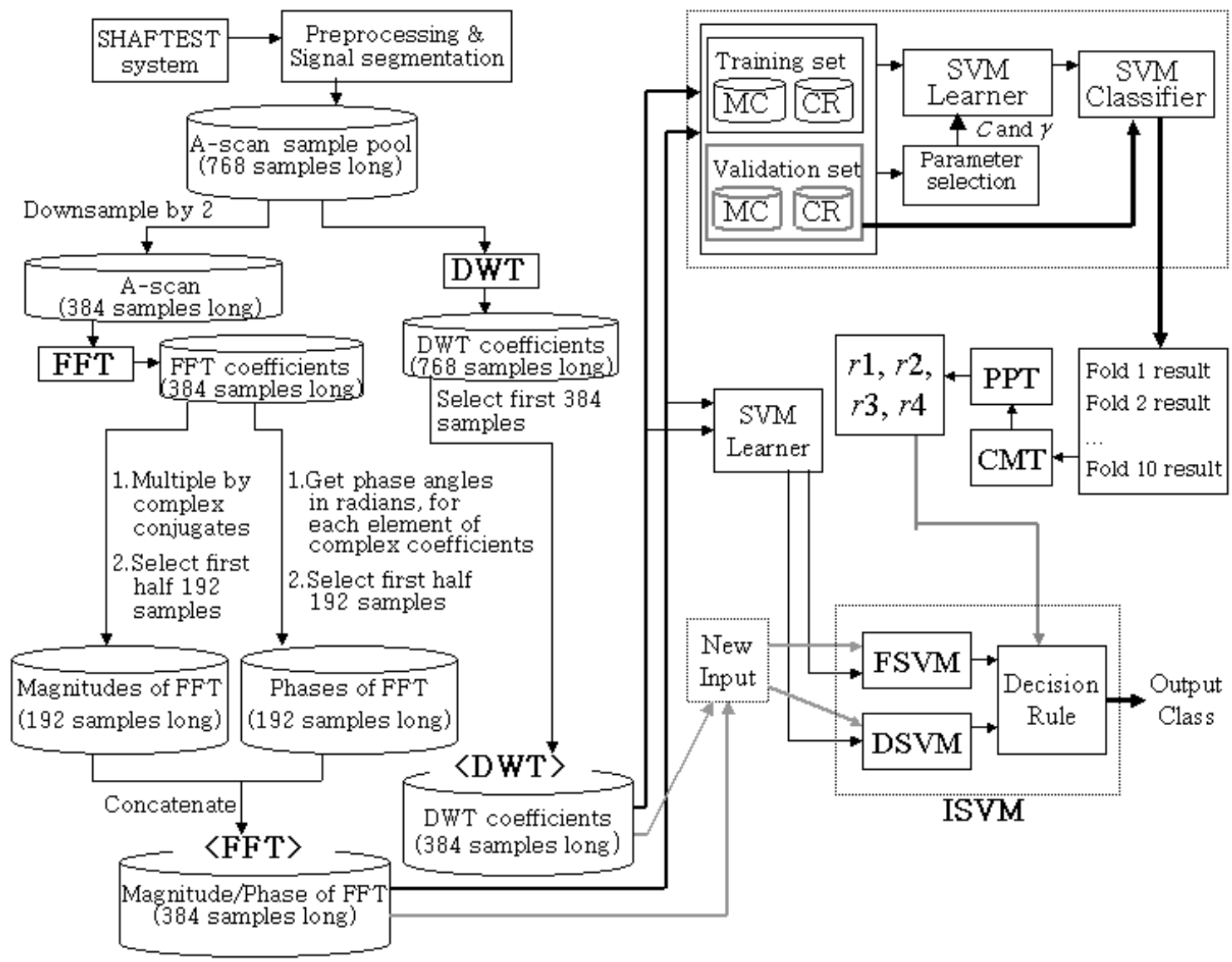

Figure 7.3: The overall procedure of our experiment for the evaluation of the performance of ISVM. 
was a primary or mode-converted echo (MC). We also used ShafTest ${ }^{T M 1}$ as a tool to capture these A-scans and build up an initial database of required echoes. For our experiment, 50 CR signals and $69 \mathrm{MC}$ signals were chosen randomly from the sample pool and they were stored as a training set. The remained samples were stored separately so that validation sets could be chosen from the remained sample pool later.

Once we had built an initial database, we mapped the time-domain data to different feature domains: frequency domain using FFT and time-frequency domain using DWT. Through this process, we generated two different sets of feature training data, which would later become the input to the SVM classifiers. In particular, the 768 values of long time-domain vectors delivered by the gate-selection preprocessing, were down-sampled into 384 values and then transformed by FFT. For the FFT, we applied "FFT_Magpha" as a FFT-based feature extraction scheme as described in Chapter 2. That is, we concatenated sequences of magnitude components and phase components into a 384 dimensional pattern vector for SVM classification.

On the other hand, the 768 values of long time-domain signal patterns were also converted by DWT. We compressed the 768 values representing the DWT coefficients into 384 samples by discarding the last 384 coefficients which we supposed did not contain much information, but mainly noise. Then we stored these 384 long vector of DWT coefficients as the DWT feature set. For our experiment, we applied Daubechies wavelets [26] for filtering in the same way as in experiments described in previous chapters of this thesis.

In our new integrated SVM classification system for shaft test, we needed to compare the posterior probability each hypothesis holds given that a specific training data has been observed when an error cost matrix is given by a user. Posterior probabilities for each case were summarized in PPM and the steps of constructing PPM are as follows. Firstly, we divided the FFT-based feature set and the DWT-based feature set each into 10 sets. In turn, we used 9 of these sets to train the SVM classifier, and classified the remaining set by the constructed SVM classifier. We employed RBF kernel for constructing SVM classifiers as the RBF kernel approach provides nonlinear mapping, requires comparatively small numbers of hyper-parameters and has fewer numerical difficulties. We repeated this process with all 10 possible combinations and collated all the classification results of ten validation sets.

\footnotetext{
${ }^{1}$ This is a trade mark of CCI Pope.
} 
Then we stored the whole concatenated classification results and the statistics of the stored result data were presented on CMT for both FFT data and DWT data. PPTs were then completed in the way explained in Table7.2 (c) and Table7.2 (d) respectively.

On the other hand, we trained two other SVM models with the FFT feature set and the DWT feature set and named them FSVM and DSVM respectively. These two models became sub-classifiers of ISVM and their individual classification performances were compared with ISVM's.

As explained previously, the decision making rule set on ISVM required five parameters: $r, r_{1}, r_{2}, r_{3}$ and $r_{4}$. All values except $r$ were calculated using values in PPT shown in Table7.2 (b) and they are 0.037, 0.925, 2.714, 68.16 respectively. With these four values, we can get five possible ranges where $r$ is assumed to be: 1) $r<0.037,2) 0.037<r<0.925$, $3) 0.925<r<2.714$ and 4$) 2.714<r<68.16$ and 5$) r>68.16$

In order to examine the performance of ISVM compared to FSVM or DSVM, we chose 23 samples randomly from the sample pool which is a sample pool remaining after the training set was selected earlier. These samples were classified by ISVM for each of the five assumptions on $r$ and error cost in each set was calculated. The error cost wass also counted on the result classified by a single FSVM or DSVM, and compared with the error cost of ISVM result. This test and comparison process were carried out 10 different times and the results are presented in Figure 7.4.

The following points are noteworthy from the experimental result.

- We see that in most scenarios, the integrated SVM classifier (ISVM) shows better classification performance than a single-feature-based classifier. That means we obtain more informative and reliable classification by combining decisions from individual classifiers.

- In the ISVM, different signal features that FFT or DWT highlights are selectively facilitated. As presented in Figure 7.4 (a), when we assume $r<r_{2}, 4$ out of 10 tests (run 1, run 3, run 5 and run 6) show that FSVM performs very much better than DSVM (run 1, run 3 and run 6) or slightly better than DSVM (run 5). In those cases, we would dismiss the outcomes of FSVM in spite of its superiority to DSVM in the 


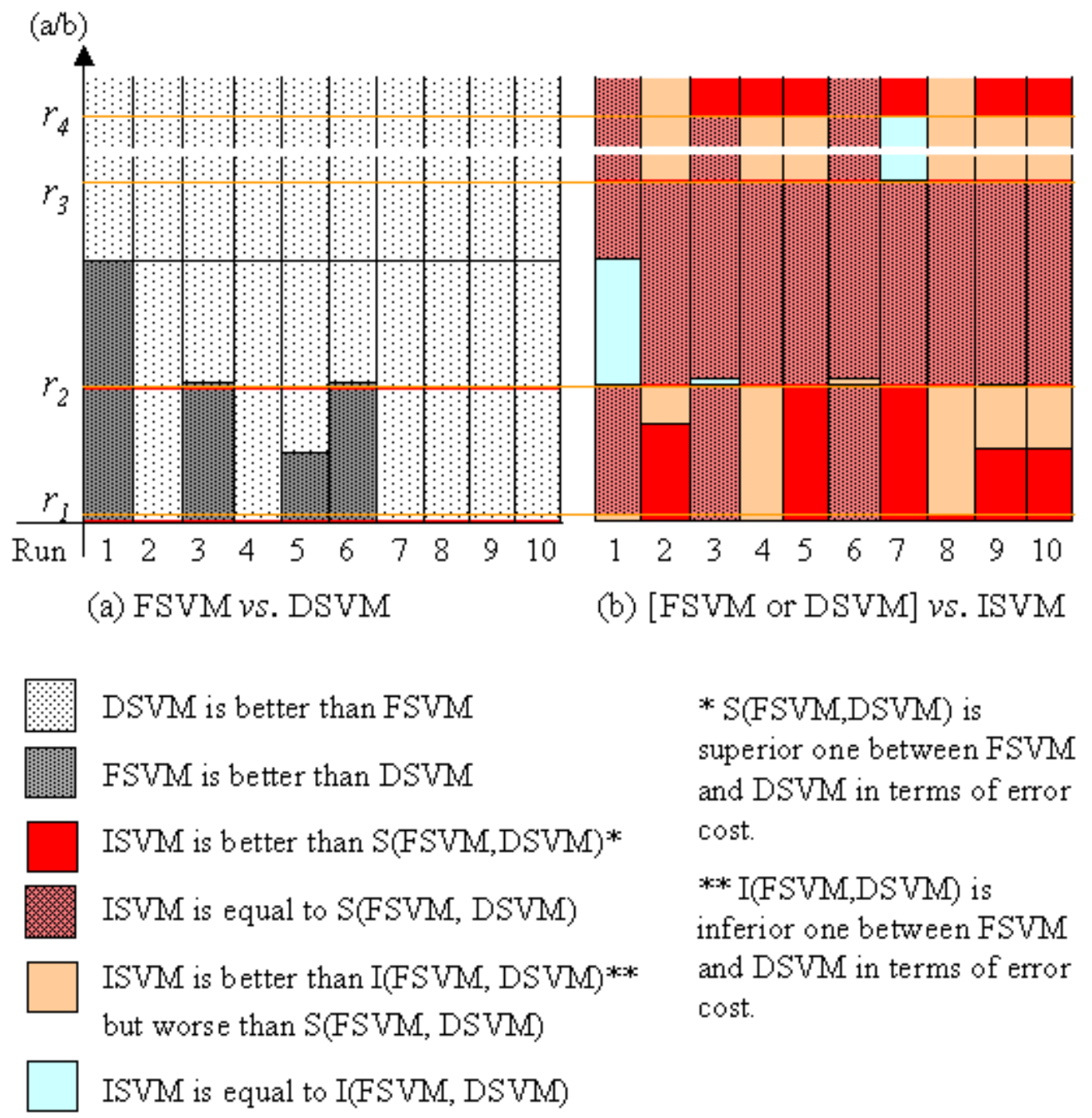

Figure 7.4: Comparing the classification performance of FSVM, DSVM and ISVM: (a)FSVM vs DSVM (b)[FSVM or DSVM] vs ISVM 
specific error-cost range, if we were using only one single classifier system. Without considering cost, we know the best overall classifier is DSVM as its overall accuracy is better than the other (FSVM). However, as seen in Figure 7.4 (b), ISVM facilitates the benefit of FSVM or DSVM selectively, resulting in better classification performance in the cost sensitive context (which is more realistic in practice).

- ISVM enhances the dynamic use of classifiers using different feature schemes in a cost-sensitive environment. As seen on Figure 7.4 (b), the superiority of ISVM to single-feature-based classifiers (FSVM and DSVM) is most recognizable in the range of $r$ is $r<r_{1}$ or $r>r_{4}$. We can integrate this kind of result as a prior knowledge when practically using the ISVM in a cost-sensitive environment, so that the ISVM can be utilized dynamically depending on the value of error-cost $(r)$.

\subsection{Remarks}

When we are to combine the results of classifiers, the machine learning literature offers schemes like boosting and bagging. However, these are mostly effective ways to enhance the predictive value of rough classifications and to re-assign weights to instances in the training set to re-focus the learning on hard instances. But, in the application at hand, there are reasons to believe that the feature extraction methods are obtaining fundamental properties of the signal (like frequency or phase information). We do not want to totally dismiss the outcomes of a classifier based on FFT even though the classifiers based on DWT have been shown to be superior.

The above motivation guided our interest for an integrated SVM classifier (ISVM), that is developed by combining and efficiently employing the strengths of each of two SVM classifiers (FSVM and DSVM) using two different feature extraction schemes FFT and DWT. Especially, in the ISVM, single classifiers are combined considering the misclassification cost of obtaining a more informative classification for its application in the field. We evaluated the ISVM by comparing its classification performance with single classifier systems. The analysis of the experiment result shows not only that the ISVM shows better classification performance than a single-feature-based classifier, but also that the ISVM enhances facil- 
itating different signal features selectively, thus enhancing the dynamic use of classifiers using different feature schemes in a cost-sensitive environment. 


\section{Chapter 8}

\section{Conclusions and Future Work}

This chapter summarizes the content of this thesis and provides future work suggested by this thesis.

\subsection{Summary}

Applications of machine learning demand exploration of feature extraction methods and classifier types in order to obtain systems with reliable high accuracy. The industrial application discussed in this thesis is the classification of ultrasonic echoes in an A-scan for testing shafts. The application is particularly challenging as A-scans are taken from the end of a long large complex shaft. Although several pattern analysis and machine learning techniques have been used with success in analyzing ultrasonic A-scan data [43, 89], they are typically in the context of very short signals. Those cases are usually much simpler; in particular, the task is reduced to detecting the existence of an echo (indicating a fault in the material). In long shafts there are many kinds of echoes, and in fact there are echoes where there is no fault. These mode-converted echoes are the result of reflection and other artifacts of the ultrasonic signal navigating and filling the shaft. They may cause misjudgement of the position of real faults (cracks) of shafts, so to distinguish them from genuine echoes is important. 
Therefore, the problem is to discriminate efficiently the different types of reflectors among the large volumes of ultrasonic shaft test data, and classify them into a) those that correspond to design features of the shaft (DF), b) those that correspond to flaws, cracks and other defects (CR) and c) the multiple reflections and mode-converted echoes (MC) of the two previous cases. Among these three causes of echoes, type DF is considered easier to distinguish than the other types. Also, in the field, the signal echoes caused by CR can be confused with fainted echoes caused by MC and vice versa. Consequences of misclassification are catastrophic with enormous cost in downtime, consequential damage to associate equipment and potential injury to personnel [23].

Modern signal processing techniques and artificial intelligence tools eliminate inconsistent results present even in classification by the same human expert. These approaches are integrated as automatic ultrasonic signal classification (AUSC) systems. In an AUSC system, ultrasonic flaw signals acquired in a form of digitized data are preprocessed and informative features are extracted using digital signal-processing techniques. The main interest for the AUSC research community has been the extraction of effective sets of features from which classification might be performed more efficiently and accurately. While it is hard to determine which set of features is best, it is important to at least identify those that make the process reliable and effective in the field. It is also important to relate some features to some understanding of the phenomena (in terms of its physics).

However, the relationship between ultrasonic signal characteristics and flaw classes is not straightforward. We need to extract an informative set of signal features which becomes the basis of decision-making for classification. Two main issues are to identify the better set of features and to identify the more suitable learning algorithm, in order to enhance the classification performance more accurately and reliably. For ultrasonic shaft signal classification the most competitive feature extraction approaches are Fast Fourier Transform (FFT) and Discrete Wavelet Transform (DWT). Artificial Neural Networks (ANN) and Support Vector Machines (SVM) are the top two approaches to build classifiers in this field.

The FFT is a useful scheme for extracting frequency-domain signal features [23, 62]. This seems natural when dealing with ultrasonic signals since the traditional representation of these types of signals is by mathematical Fourier series that identify physically meaningful features, like frequency and phase. In most previous AUSC-related studies, the researchers 
extracted frequency-domain features through the FFT scheme and then applied an extra process for selecting a set of features in order to use them as input vectors to the classifier. However, our interest in this thesis is to use directly the whole signal section derived from the ultrasonic scans, employing a minimum of preprocessing, using only the FFT technique as inputs into the classifier. On the other hand, more recent studies on the ultrasonic flaw classification employ the DWT as part of their feature extraction scheme since DWT provides effective signal compression and time-frequency presentation $[69,86]$.

In their quest for better sets of features for AUSC, many researchers have made a comparison between these two feature extraction schemes (FFT and DWT). More specifically, they commonly employed two different preprocessing techniques using coefficients of FFT and coefficients of DWT in order to extract feature sets, and compared the classification performance using each feature set. Most results of comparing FFT and DWT showed a superiority of DWT over FFT in discriminating the type of flaw (or its non-existence) in the context of comparatively shorter and simpler signals $[74,78,90]$. This is very different in long shafts where the issue is to determine if an echo is indeed a flaw or a mode-converted echo. This thesis is the first study analyzing feature extraction in more complex ultrasonic signals for testing long shafts where mode-converted echoes are the main objects to be discriminated.

The subject of many studies including this thesis is to investigate which feature extraction scheme between FFT and DWT performs best at extracting features in ultrasonic signals. The claims in previous studies from similar scenarios on the superiority of DWT coefficients to FFT coefficients as features for the ultrasonic signal classification is subject to debate. The reason is that most previous comparison studies used only the magnitude component of the transformed signals using FFT and their phase components were naturally excluded through the process of using FFT coefficients as feature vectors for classifiers (we named this type of FFT coefficients "FFT_Mag" in Chapter 2). Therefore, in order to make a fairer comparison, we formed a new set of FFT feature vectors which effectively represent both magnitude and phase information of FFT sequences (we named this type of FFT coefficients "FFT_Magpha"). In Chapter 3 of this thesis, through the experimental comparison and analysis of the classification performances of using "FFT_Mag" and "FFT_Magpha" respectively as feature vectors to classifiers, we showed "FFT_Magpha" is a more efficient FFT 
based feature extraction approach than "FFT_Mag" while using exactly the same dimensionality reduction. This means we have a state-of-the-art FFT method for our application. Because of the success with this design of FFT feature vector ("FFT_Magpha"), as the most effective FFT variants, "FFT_Magpha" is the one we use for comparison between FFT and DWT as feature extraction schemes for the classification of ultrasonic shaft signals.

After features are chosen, classifiers are applied to classify signals. Among a number of classification algorithms proposed in previous studies, ANNs have been most popularly employed due to their ability to make non-linear decision boundaries. Especially, ANNs using the Backpropagation algorithm have gained more popularity and are now considered to outperform conventional pattern recognition algorithms or other ANNs for ultrasonic signal classification [62]. We also employed ANNs using the Backpropagation algorithm to compare two feature extraction techniques FFT and DWT and the study established experimentally that DWT had stronger potential as a feature extraction scheme for feeding ANNs. It showed that training data could be selected with higher flexibility and then used in these networks for in-field ultrasonic shaft signal classification.

However, this raised the issue about the synergy created by the DWT as feature selector and the ANN as classifier: namely, is this a feature extraction that is too much well fit for ANN and not useful for other classifiers? In addition, considering the many difficulties inherent in the ANN learning paradigm such as generalization control, overfitting and parameter tuning, we remain more conservative about DWT's predominance. For this reason, we made a new comparative experiment involving SVM instead of ANN models in Chapter 4. The reported results confirmed that the DWT is indeed the superior feature extraction scheme in the classification of echoes from ultrasonic signals in long shafts, because the statistical properties of SVM indicate robustness in its construction, especially when a limited number of training examples are available.

Though we demonstrated the potential for using DWT as a useful feature for input into classifiers in the case of ultrasonic shaft test data, DWT exhibits a time-variance problem that has resulted in reservations about its wide acceptance. To overcome this problem, we developed a new technique to derive a preprocessing method for time-domain A-Scan signals. This technique offers consistent extraction of a segment of the signal from long signals that occur in the non-destructive testing of shafts. We supplied classifiers using 
ANNs and SVMs with features generated by our new preprocessing method and compared and evaluated their classification performance. We also compared the performance of the newly-developed technique with other alternatives and reported the experimental results in Chapter 5. This investigation empirically establishes that DWT coefficients can be used as a feature extraction scheme more reliably by applying our new preprocessing technique.

While other chapters of this thesis focus on finding the best single classifier model by determining the best selected feature extraction scheme (FFT or DWT), in Chapter 6 and Chapter 7, we learn multiple models of the shaft test data and combine their outputs for making a final decision for classification. The reason for combining multiple models (ensemble of classifiers) constructed by a single feature extraction scheme (FFT or DWt) and a single learning algorithm (ANN or SVM) is that FFT might reflect physical properties that are different from those that DWT shows. We suspect that including the FFT as another informant of the decision process, even if the accuracy using DWT has shown to be superior, should improve accuracy.

There are various approaches (homogeneous or heterogeneous) for generating multiple classifiers and for combining the outputs of multiple models [29, 98]. The issue raised by this diversity of methods for generating and combining multiple models is to pursue the best generation-combination method for constructing the most effective and reliable multi-model AUSC system for our application domain. Therefore, in order to construct an effective multiclassifier system, we need to decide a scheme for creating models and also a combination method for decision making. In Chapter 6, we explore the diverse possibilities of heterogeneous and homogeneous ensembles, combining techniques, feature extraction methods and classifier types, and determine guidelines for the construction of an integrated multiclassifier model using both feature schemes (FFT and DWT) effectively. We also analyze the type of errors on each combination model in order to gain insight into appropriate combining strategies. The experimental results analyzed in Chapter 6 imply that combining features in general improves the classification accuracy. Based on this result, we also confirm the observation that there seem to be uncorrelated errors among the variants explored in previous chapters of this thesis, and therefore an ensemble of classifiers has the potential for improvement in discriminating ultrasonic shaft test signals.

In Chapter 7, we design a cost-sensitive combined classification system named ISVM, which 
is constructed by a homogeneous approach efficiently employing the strengths of each of two SVM classifiers (FSVM and DSVM) using two different feature extraction schemes (FFT and DWT). The analysis of the experimental results presented in Section 6.4 indicates that different feature sets become more advantageous when we use the SVM as a learning algorithm than when we use ANNs. We thus employ SVM for the system in Chapter 7. We combine multiple classifiers in the ISVM especially considering the misclassification cost to obtain a more informative classification for its application in the field. This is meaningful because the field of shaft inspection data can be in a cost-sensitive environment where we need to predict whether a signal echo is caused by $\mathrm{CR}$ (crack) or $\mathrm{MC}$ (mode-conversion) and two different costs can be assigned, each for two misclassification cases. These two different error costs become a basic component for constructing the decision-making rule in ISVM. Consequently it becomes a cost-sensitive classification system. We compare the classification performance of ISVM with each single classifier FSVM or DSVM through the experiment presented in Chapter 7. The experimental result shows that we obtain more informative and reliable classification by combining multi-classifiers' decisions than by depending on the classification decision by a single classifier system.

\subsection{Future Work}

As presented in Figure 1.5 in Chapter 1, we can divide our work presented in this thesis roughly into three categories. They are related to the three steps of processes in an AUSC system, which are preprocessing, feature extraction and classification. The advances presented in this thesis can be continued in several directions for future work as follows. We also categorize them according to the necessary steps to construct an AUSC system, and summarize them in Figure 8.1.

\section{- Ultrasonic signal data collection}

- Ultrasonic signals can be presented to the operator in several formats. For the empirical work in this thesis, we collected ultrasonic signal data in the form of an A-scan which is a plot of signal voltage versus time for one transducer position. From the time of an echo, the distance can be computed stating that it is equal 


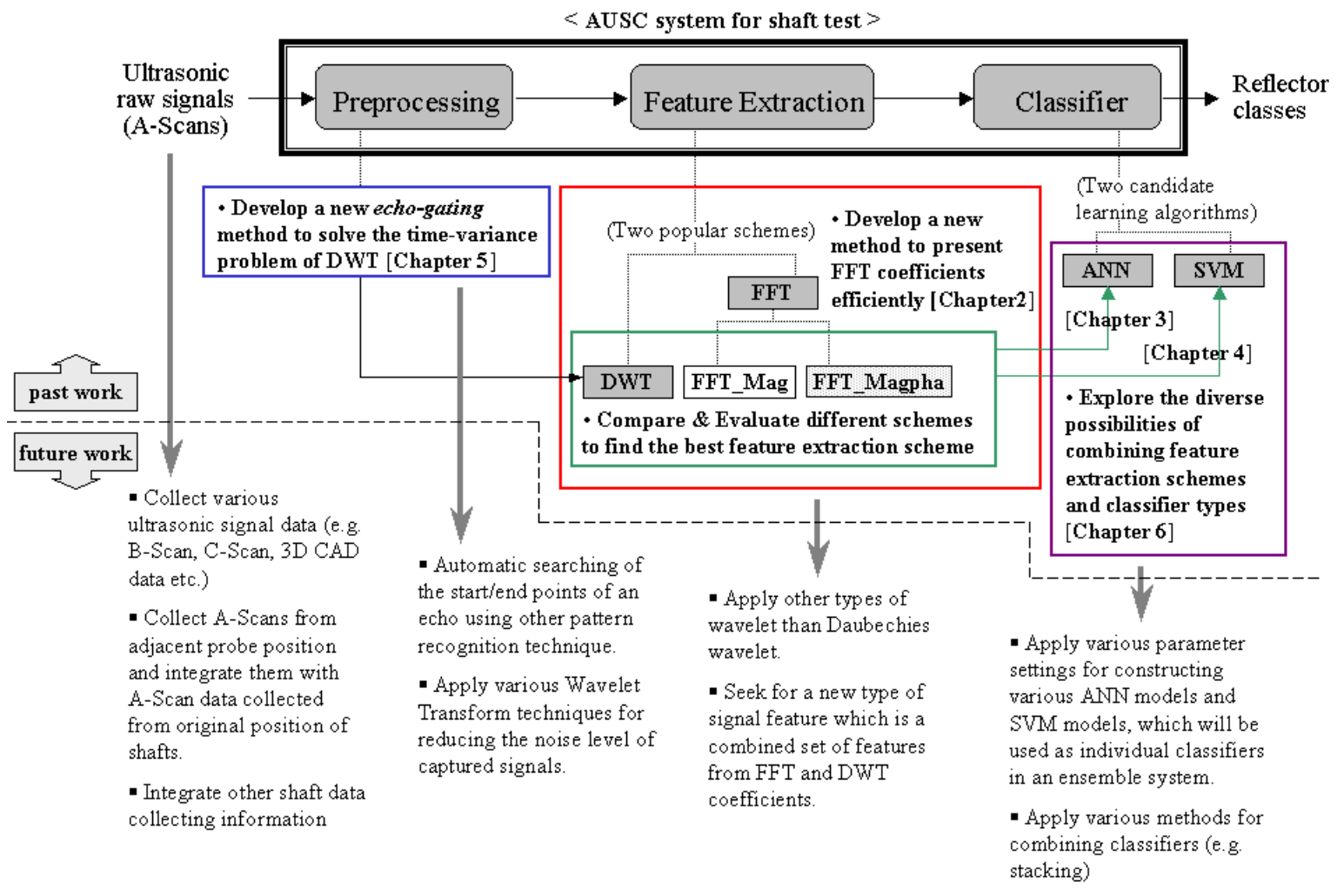

Figure 8.1: A summary of the possible future extensions of this work. 
to wave velocity by time of flight. In addition to A-Scans, there are two other modes; B-scans and C-scans. In the B-scan mode, one axis of the display is proportional to the transducer position and the other axis is proportional to the time delay of the A-scan. It represents a lateral view of the object inspected according to the direction of scan. In the $\mathrm{C}$-scan mode, the two axes of the display are proportional to the transducer position following the direction of scan and its perpendicular direction. It corresponds to the plan view of the object inspected. In this mode, the signal is represented color coded proportional to its amplitude. Moreover, we can have a CAD model of the object as an extended domain in which we are applying ultrasonic flaw detection. In this method, ray -tracing is used in computer graphics to model the paths of rays of light in space. This raytracing will be used to model the paths of the ultrasonic waves inside the body of the object as it bounces on walls and design features. By integrating these kinds of extra presentation modes for ultrasonic signals, we may be provided additional domain knowledge that could significantly assist the classifiers. We particularly expect that the additional signal data achieved by adjacent positioning of the transducer will play a more important role in assisting the classifier.

\section{- Preprocessing}

- We introduced a new echo-gating technique in Chapter 5 as an approach to overcome the time-invariance problem of DWT coefficients. In the process of applying this new method, we need to single out only an echo from the whole A-scan signal in order to gate exactly the segment that includes the echo energy, excluding exactly the background noise grass. Thus, we need to decide when (in the time dimension) the echo starts and at which point it ends. We suggested a specific decision rule for finding the starting point and the ending point for an echo in this thesis. However, rather than depending on a decision rule made up by one specification, we may also apply an automatic searching method for the starting or ending point of an echo by using various pattern recognition techniques.

- To achieve a reliable method for extracting the segment where the echo's energy is manifested, the background noise needs to be treated properly since it is usually distributed irregularly on the entire A-scan. In order to deal with 
this noise problem, we applied a thresholding method by which we calculated a thresholding parameter and used it for filtering noise from the original A-scan. Wavelet analysis is known to be able to compress or de-noise a signal without appreciable degradation, because it affords a different view of data than those presented by traditional techniques. Therefore, further study is needed to apply various wavelet techniques to suppress the background noise for our application.

\section{- Feature extraction}

- Among various signal features based on time-domain or frequency-domain, we mainly discussed the top two approaches FFT and DWT as feature extraction schemes for our application. Firstly, we investigated which feature extraction scheme produced better classification performance, by comparing classification results of two separate classifiers which were trained by either feature vector. Secondly, we combined two different classifiers, constructed by one or another of the feature extraction schemes: FFT or DWT, in order to make a final classification decision. These two types of trials were inspired by a preparatory experiment (not presented in this thesis) in which we concatenated both feature vectors to consist of one feature vector and then applied it to construct a classifier. The experimental result did not show any benefits for the classification performance compared to the performance of a single classifier based on FFT or DWT features. This empirical result implies that the efficiency of the classifier is diminished by concatenating two feature vectors. We are unclear about the reasons for this. A possible reason is that there are a range of duplicated features between FFT-based features and DWT-based features. Therefore, we need further studies to seek for a new type of signal feature which is a combined set of features from FFT and DWT coefficients. We will need to apply an advanced feature selection technique to achieve this.

- Another possible extension of our current study related to feature extraction for our application is to apply various wavelets for getting DWT-based features. Since the methods of wavelet analysis were first developed, the wavelet domain has been growing very quickly and various types of wavelet families have been developed. Among those various wavelets, we applied Daubechies wavelet in the process of getting DWT coefficients as it is known to be most representative 
wavelet making discrete wavelet analysis practicable. For further studies, we need to apply different types of wavelet families whose qualities vary according to different criteria.

\section{- Classification and Decision Making}

- The selection of architecture or parameters for constructing ANN models for experiments in this thesis was originally based on the experimental results from a previous study [23]. In this previous study, the researchers tried to design a number of different architectures of ANN models for classifying shaft test data, and analyzed the efficiency of each model in terms of the generalization ability and the classification performance. In selecting a kernel of SVM used in experiments in this thesis, we chose Radial Basis Function (RBF) as the RBF kernel is generally known to provide nonlinear mapping, to require comparatively small numbers of hyper-parameters and to have fewer numerical difficulties. In most experiments presented in this thesis, these parameter selections for ANN models or kernel selection for SVM models were employed for comparing SVM-based classifiers vs. ANN-based classifiers or FFT-based classifiers vs. DWT-based classifiers in order to identify which single classifier system is better for ultrasonic signal classification for shaft tests (refer to Chapter 3, Chapter 4 and Chapter 5). Motivated by these experimental results, we applied the same methods for selecting architectures, parameters and kernels for ANN models or SVM models which were employed as individual classifiers for a combined classifier system (refer to Chapter 6 and Chapter 7). In order to develop a more advanced and extensive ensemble system for our application, we need to apply an extensive variety (such as parameter settings and architecture selection for ANNs or kernel selection for SVMs) to construct single classifier models which will be members of a multi-classifier system.

- For the experimental comparison in Chapter 6 where we explored the combining of classifiers along the dimension of feature extraction mechanism, along the dimension of combination methods and along the dimension of types of classifiers, we applied three generally known combining methods. In addition to these methods, many methods have been explored to combine individual classification decisions in an ensemble of classifiers [29]. They include various weighted or un- 
weighted voting methods, the Stacking method or the Cascading approach. The Stacking [101] approach, in particular, has gained more attention recently as a method to combine classifiers generated by applying different learning algorithms. In the stacking method, a learning algorithm is used to learn how to combine the predictions of the base-level classifiers. This is based on meta-learning which has been proposed to deal with the issues of algorithm selection [8,39]. Thus the induced meta-level classifier is used to obtain the final prediction from the predictions of the base-level classifiers.

A possible extension on our investigational studies presented in Chapter 6 is applying other combining methods like stacking to design a more advanced multiclassifier system for testing ultrasonic shaft signals. In the experiment presented in Chapter 6, we only combined the class prediction made by each individual classifier. In order to apply a stacking approach more efficiently, we can use a more varied set (not only using the prediction of class) of decision factors to build a meta-level data set. To prepare for such extensional future work, we selected a topology of ANNs with two output nodes so that the final weights of the nodes can be used as members of a meta-level data set. In a similar way, the final weight values obtained by SVMs also can be used as a meta-level data. 
Appendix A

ANOVA test result 


\section{A.1 ANOVA test for the experimental results of Chapter 3}

\begin{tabular}{|c|c|c|c|c|c|c|}
\hline \multirow{2}{*}{\multicolumn{7}{|c|}{$\begin{array}{l}\text { Epoch } \\
\text { ANOVA: Single Factor }(p=0.05)\end{array}$}} \\
\hline & & & & & & \\
\hline \multicolumn{7}{|l|}{ SUMMARY } \\
\hline Groups & Count & Sum & Average & Variance & & \\
\hline DWT & 10 & 143325.01 & 14332.501 & 27422832 & & \\
\hline FFT Mag & 10 & 964250 & 96425 & $3.44 \mathrm{E}+09$ & & \\
\hline FFT MagPha & 10 & 188988.22 & 18898.822 & $1.93 E+08$ & & \\
\hline \multicolumn{7}{|l|}{ ANOVA } \\
\hline Source of Variation & $S S$ & $d f$ & MS & $F$ & P-value & $F_{\text {crit }}$ \\
\hline Between Groups & $4.2568 \mathrm{E}+10$ & 2 & $2.128 \mathrm{E}+10$ & 17.46597 & $1.36 \mathrm{E}-05$ & 3.354131 \\
\hline Within Groups & $3.2902 \mathrm{E}+10$ & 27 & 1.219E +09 & & & \\
\hline Total & $7.547 \mathrm{E}+10$ & 29 & & & & \\
\hline \multirow{2}{*}{\multicolumn{7}{|c|}{ Accuracy $(\%)$}} \\
\hline & & & & & & \\
\hline \multicolumn{7}{|c|}{ ANOVA: Single Factor $(p=0.05)$} \\
\hline & & & & & & \\
\hline \multicolumn{7}{|l|}{ SUMMARY } \\
\hline Groups & Count & Sum & Average & Variance & & \\
\hline DWT & 10 & 916.16481 & 91.616481 & 0.615267 & & \\
\hline FFT_Mag & 10 & 901.53001 & 90.153001 & 0.838981 & & \\
\hline FFT MagPha & 10 & 913.62323 & 91.362323 & 1.186149 & & \\
\hline \multicolumn{7}{|l|}{ ANOVA } \\
\hline Source of Variation & SS & $d f$ & MS & $F$ & P-value & $F_{\text {crit }}$ \\
\hline Between Groups & 12.2294331 & 2 & 6.1147166 & 6.947494 & 0.00368 & 3.354131 \\
\hline Within Groups & 23.7635823 & 27 & 0.8801327 & & & \\
\hline Total & 35.9930154 & 29 & & & & \\
\hline
\end{tabular}




\section{A.2 ANOVA test for the experimental results of Chapter 4}

\begin{tabular}{|c|c|c|c|c|c|c|}
\hline FFT & & & & & & \\
\hline \multicolumn{7}{|c|}{ ANOVA: Single Factor $(p=0.05)$} \\
\hline \multirow{2}{*}{\multicolumn{7}{|c|}{ SUMMARY }} \\
\hline & & & & & & \\
\hline Groups & Count & Sum & Average & Variance & & \\
\hline CR & 10 & 671.77 & 67.177 & 74.01262 & & \\
\hline $\mathrm{MC}$ & 10 & 925.2 & 92.52 & 63.39733 & & \\
\hline \multicolumn{7}{|l|}{ ANOVA } \\
\hline Source of Variation & SS & $d f$ & MS & $F$ & $P$-value & $F_{\text {crit }}$ \\
\hline Between Groups & 3211.33825 & 1 & 3211.3382 & 46.74098 & $2.13 \mathrm{E}-06$ & 4.413863 \\
\hline Within Groups & 1236.68961 & 18 & 68.704978 & & & \\
\hline Total & 4448.02786 & 19 & & & & \\
\hline & & & & & & \\
\hline \multicolumn{7}{|l|}{ DWT } \\
\hline \multicolumn{7}{|c|}{ ANOVA: Single Factor $(p=0.05)$} \\
\hline & & & & & & \\
\hline \multicolumn{7}{|l|}{ SUMMARY } \\
\hline Groups & Count & Sum & Average & Variance & & \\
\hline CR & 10 & 931 & 93.1 & 34.43778 & & \\
\hline $\mathrm{MC}$ & 10 & 835 & 83.5 & 59.42222 & & \\
\hline \multicolumn{7}{|l|}{ ANOVA } \\
\hline Source of Variation & SS & $d f$ & MS & $F$ & P-value & F crit \\
\hline Between Groups & 460.8 & 1 & 460.8 & 9.818879 & 0.005742 & 4.413863 \\
\hline Within Groups & 844.74 & 18 & 46.93 & & & \\
\hline Total & 1305.54 & 19 & & & & \\
\hline
\end{tabular}




\begin{tabular}{|c|c|c|c|c|c|c|}
\hline CR & & & & & & \\
\hline ANOVA: Single Fac & $\operatorname{tor}(p=0.05)$ & & & & & \\
\hline SUMMARY & & & & & & \\
\hline Groups & Count & Sum & Average & Variance & & \\
\hline FFT & 10 & 671.77 & 67.177 & 74.01262 & & \\
\hline DWT & 10 & 931 & 93.1 & 34.43778 & & \\
\hline ANOVA & & & & & & \\
\hline Source of Variation & $S S$ & $d f$ & MS & $F$ & $P$-value & $F_{\text {crit }}$ \\
\hline Between Groups & 3360.00964 & 1 & 3360.0096 & 61.96399 & 3.08E-07 & 4.413863 \\
\hline Within Groups & 976.05361 & 18 & 54.225201 & & & \\
\hline Total & 4336.06325 & 19 & & & & \\
\hline & & & & & & \\
\hline TIL & & & & & & \\
\hline ANOVA: Single Fad & tor $(p=0.05)$ & & & & & \\
\hline SUMMARY & & & & & & \\
\hline Groups & Count & Sum & Average & Variance & & \\
\hline FFT & 10 & 925.2 & 92.52 & 63.39733 & & \\
\hline DWT & 10 & 835 & 83.5 & 59.42222 & & \\
\hline ANOVA & & & & & & \\
\hline Source of Variation & $S S$ & $d f$ & MS & $F$ & P-value & $F_{\text {crit }}$ \\
\hline Between Groups & 406.802 & 1 & 406.802 & 6.624385 & 0.019123 & 4.413863 \\
\hline Within Groups & 1105.376 & 18 & 61.409778 & & & \\
\hline Total & 1512.178 & 19 & & & & \\
\hline & & & & & & \\
\hline Overall & & & & & & \\
\hline ANOVA: Single Fao & tor $(p=0.05)$ & & & & & \\
\hline & & & & & & \\
\hline SUMMARY & & & & & & \\
\hline Groups & Count & Sum & Average & Variance & & \\
\hline FFT & 10 & 789.63865 & 78.963865 & 45.84841 & & \\
\hline DWT & 10 & 866.86905 & 86.686905 & 14.99048 & & \\
\hline ANOVA & & & & & & \\
\hline Source of Variation & $S S$ & $d f$ & MS & $F$ & P-value & $F_{c r i t}$ \\
\hline Between Groups & 298.226714 & 1 & 298.22671 & 9.803819 & 0.005773 & 4.413863 \\
\hline Within Groups & 547.54999 & 18 & 30.419444 & & & \\
\hline Total & 845.776704 & 19 & & & & \\
\hline
\end{tabular}




\section{A.3 ANOVA test for the experimental results of Chapter 5}

\begin{tabular}{|c|c|c|c|c|c|c|}
\hline \multirow{2}{*}{\multicolumn{7}{|c|}{$\begin{array}{l}\text { ANN-DWT } \\
\text { ANOVA: Single Factor }(p=0.05)\end{array}$}} \\
\hline & & & & & & \\
\hline & & & & & & \\
\hline \multicolumn{7}{|l|}{ SUMMARY } \\
\hline Groups & Count & Sum & Average & Variance & & \\
\hline RA & 10 & 523.9 & 52.39 & 58.51878 & & \\
\hline $\mathrm{CP}$ & 10 & 826.5 & 82.65 & 87.72056 & & \\
\hline MIE & 10 & 759.8 & 75.98 & 61.99733 & & \\
\hline So & 10 & 776.4 & 77.64 & 30.44711 & & \\
\hline SZ & 10 & 875.7 & 87.57 & 40.54233 & & \\
\hline \multicolumn{7}{|l|}{ ANOVA } \\
\hline Source of Variation & SS & $d f$ & MS & $F$ & P-value & $F_{\text {crit }}$ \\
\hline Between Groups & 7353.6692 & 4 & 1838.4173 & 32.91987 & $7.68 \mathrm{E}-13$ & 2.578739 \\
\hline Within Groups & 2513.035 & 45 & 55.845222 & & & \\
\hline Total & 9866.7042 & 49 & & & & \\
\hline & & & & & & \\
\hline \multicolumn{7}{|c|}{ ANN-CDWT } \\
\hline \multicolumn{7}{|c|}{ ANOVA: Single Factor $(p=0.05)$} \\
\hline & & & & & & \\
\hline \multicolumn{7}{|l|}{ SUMMARY } \\
\hline Groups & Count & Sum & Average & Variance & & \\
\hline RA & 10 & 727.6 & 72.76 & 46.86933 & & \\
\hline $\mathrm{CP}$ & 10 & 839.2 & 83.92 & 27.25733 & & \\
\hline ME & 10 & 737.1 & 73.71 & 27.80544 & & \\
\hline So & 10 & 850.9 & 85.09 & 20.87211 & & \\
\hline $\mathbf{S Z}$ & 10 & 883.3 & 88.33 & 14.88011 & & \\
\hline \multicolumn{7}{|l|}{ ANOVA } \\
\hline Source of Variation & SS & $d f$ & MS & $F$ & $P$-value & $F_{\text {crit }}$ \\
\hline Between Groups & 1997.4188 & 4 & 499.3547 & 18.13404 & $6.18 \mathrm{E}-09$ & 2.578739 \\
\hline Within Groups & 1239.159 & 45 & 27.536867 & & & \\
\hline Total & 3236.5778 & 49 & & & & \\
\hline
\end{tabular}




\begin{tabular}{|l|r|r|r|r|r|r|}
\hline \multicolumn{1}{|l|}{ SVM-DWT } & & & & & \\
\hline ANOVA: Single Factor ( $\mathrm{p}=0.05)$ & & & & \\
\hline
\end{tabular}




\begin{tabular}{|c|c|c|c|c|c|c|}
\hline ANN-RA & & & & & & \\
\hline \multicolumn{7}{|c|}{ ANOVA: Single Factor $(p=0.05)$} \\
\hline & & & & & & \\
\hline \multicolumn{7}{|l|}{ SUMMARY } \\
\hline Groups & Count & Sum & Average & Variance & & \\
\hline DWT & 10 & 523.9 & 52.39 & 58.51878 & & \\
\hline CDWT & 10 & 727.6 & 72.76 & 46.86933 & & \\
\hline \multicolumn{7}{|l|}{ ANOVA } \\
\hline Source of Variation & SS & $d f$ & MS & $F$ & $P$-value & $F_{\text {crit }}$ \\
\hline Between Groups & 2074.6845 & 1 & 2074.6845 & 39.37227 & $6.45 \mathrm{E}-06$ & 4.413873 \\
\hline Within Groups & 948.493 & 18 & 52.694056 & & & \\
\hline Total & 3023.1775 & 19 & & & & \\
\hline & & & & & & \\
\hline \multicolumn{7}{|c|}{ ANN-CP } \\
\hline \multicolumn{7}{|c|}{ ANOVA: Single Factor $(p=0.05)$} \\
\hline \multirow{2}{*}{\multicolumn{7}{|c|}{ SUMMARY }} \\
\hline & & & & & & \\
\hline Groups & Count & Sum & Average & Variance & & \\
\hline DWT & 10 & 826.5 & 82.65 & 87.72056 & & \\
\hline CDWT & 10 & 839.2 & 83.92 & 27.25733 & & \\
\hline \multicolumn{7}{|l|}{ ANOVA } \\
\hline Source of Variation & SS & $d f$ & MS & $F$ & P-value & $F_{\text {crit }}$ \\
\hline Between Groups & 8.0645 & 1 & 8.0645 & 0.140279 & 0.71238 & 4.413873 \\
\hline Within Groups & 1034.801 & 18 & 57.488944 & & & \\
\hline Total & 1042.8655 & 19 & & & & \\
\hline & & & & & & \\
\hline \multicolumn{7}{|c|}{\begin{tabular}{l|l} 
ANN-ME \\
\end{tabular}} \\
\hline \multicolumn{7}{|c|}{ ANOVA: Single Factor $(p=0.05)$} \\
\hline & & & & & & \\
\hline \multicolumn{7}{|l|}{ SUMMARY } \\
\hline Groups & Count & Sum & Average & Variance & & \\
\hline DWT & 10 & 759.8 & 75.98 & 61.99733 & & \\
\hline CDWT & 10 & 737.1 & 73.71 & 27.80544 & & \\
\hline \multicolumn{7}{|l|}{ ANOVA } \\
\hline Source of Variation & $S S$ & $d f$ & MS & $F$ & P-value & $F_{\text {crit }}$ \\
\hline Between Groups & 25.7645 & 1 & 25.7645 & 0.573802 & 0.458555 & 4.413873 \\
\hline Within Groups & 808.225 & 18 & 44.901389 & & & \\
\hline Total & 833.9895 & 19 & & & & \\
\hline
\end{tabular}




\begin{tabular}{|c|c|c|c|c|c|c|}
\hline ANN-SO & & & & & & \\
\hline ANOVA: Single Fac & tor $(p=0.05)$ & & & & & \\
\hline & & & & & & \\
\hline SUMMARY & & & & & & \\
\hline Groups & Count & Sum & Average & Variance & & \\
\hline DWT & 10 & 776.4 & 77.64 & 30.44711 & & \\
\hline CDWT & 10 & 850.9 & 85.09 & 20.87211 & & \\
\hline ANOVA & & & & & & \\
\hline Source of Variation & $S S$ & $d f$ & MS & $F$ & P-value & $F_{\text {crit }}$ \\
\hline Between Groups & 277.5125 & 1 & 277.5125 & 10.81515 & 0.004082 & 4.413873 \\
\hline Within Groups & 461.873 & 18 & 25.659611 & & & \\
\hline Total & 739.3855 & 19 & & & & \\
\hline & & & & & & \\
\hline ANN-SZ & & & & & & \\
\hline ANOVA: Single Fad & tor $(p=0.05)$ & & & & & \\
\hline & & & & & & \\
\hline SUMMARY & & & & & & \\
\hline Groups & Count & Sum & Average & Variance & & \\
\hline DWT & 10 & 875.7 & 87.57 & 40.54233 & & \\
\hline CDWT & 10 & 883.3 & 88.33 & 14.88011 & & \\
\hline ANOVA & & & & & & \\
\hline Source of Variation & SS & $d f$ & MS & $F$ & P-value & $F_{\text {crit }}$ \\
\hline Between Groups & 2.888 & 1 & 2.888 & 0.104218 & 0.750546 & 4.413873 \\
\hline Within Groups & 498.802 & 18 & 27.711222 & & & \\
\hline Total & 501.69 & 19 & & & & \\
\hline & & & & & & \\
\hline SVM-RA & & & & & & \\
\hline ANOVA: Single Fac & tor $(p=0.05)$ & & & & & \\
\hline & & & & & & \\
\hline SUMMARY & & & & & & \\
\hline Groups & Count & Sum & Average & Variance & & \\
\hline DWT & 10 & 714.6 & 71.46 & 39.00656 & & \\
\hline CDWT & 10 & 799.98 & 79.998 & 82.24904 & & \\
\hline ANOVA & & & & & & \\
\hline Source of Variation & SS & $d f$ & MS & $F$ & P-value & $F_{\text {crit }}$ \\
\hline Between Groups & 364.48722 & 1 & 364.48722 & 6.011883 & 0.024646 & 4.413873 \\
\hline Within Groups & 1091.30036 & 18 & 60.627798 & & & \\
\hline Total & 1455.78758 & 19 & & & & \\
\hline
\end{tabular}




\begin{tabular}{|c|c|c|c|c|c|c|}
\hline \multirow{2}{*}{\multicolumn{7}{|c|}{$\begin{array}{l}\text { SVM-CP } \\
\text { ANOVA: Single Factor }(p=0.05)\end{array}$}} \\
\hline & & & & & & \\
\hline \multirow{2}{*}{\multicolumn{7}{|c|}{ SUMMARY }} \\
\hline & & & & & & \\
\hline Groups & Count & Sum & Average & Variance & & \\
\hline DWT & 10 & 893.9 & 89.39 & 54.75211 & & \\
\hline CDWT & 10 & 861.38 & 86.138 & 83.84122 & & \\
\hline \multicolumn{7}{|l|}{ ANOVA } \\
\hline Source of Variation & $S S$ & $d f$ & MS & $F$ & P-value & $F_{\text {crit }}$ \\
\hline Between Groups & 52.87752 & 1 & 52.87752 & 0.76306 & 0.393878 & 4.413873 \\
\hline Within Groups & 1247.33996 & 18 & 69.296664 & & & \\
\hline Total & 1300.21748 & 19 & & & & \\
\hline \multirow{2}{*}{\multicolumn{7}{|c|}{\begin{tabular}{l|l} 
SVM-ME & \\
\end{tabular}}} \\
\hline & & & & & & \\
\hline \multicolumn{7}{|c|}{ ANOVA: Single Factor $(p=0.05)$} \\
\hline \multirow{2}{*}{\multicolumn{7}{|c|}{ SUMMARY }} \\
\hline & & & & & & \\
\hline Groups & Count & Sum & Average & Variance & & \\
\hline DWT & 10 & 823.47 & 82.347 & 40.30942 & & \\
\hline CDWT & 10 & 851.4 & 85.14 & 82.25767 & & \\
\hline \multicolumn{7}{|l|}{ ANOVA } \\
\hline Source of Variation & SS & $d f$ & MS & $F$ & P-value & $F_{\text {crit }}$ \\
\hline Between Groups & 39.004245 & 1 & 39.004245 & 0.636455 & 0.435396 & 4.413873 \\
\hline Within Groups & 1103.10381 & 18 & 61.283545 & & & \\
\hline Total & 1142.10806 & 19 & & & & \\
\hline & & & & & & \\
\hline \multicolumn{7}{|c|}{ SVM-SO } \\
\hline \multicolumn{7}{|c|}{ ANOVA: Single Factor $(p=0.05)$} \\
\hline & & & & & & \\
\hline \multicolumn{7}{|l|}{ SUMMARY } \\
\hline Groups & Count & Sum & Average & Variance & & \\
\hline DWT & 10 & 916.17 & 91.617 & 15.88078 & & \\
\hline CDWT & 10 & 916.7 & 91.67 & 18.99789 & & \\
\hline \multicolumn{7}{|l|}{ ANOVA } \\
\hline Source of Variation & SS & $d f$ & MS & $F$ & P-value & $F_{\text {crit }}$ \\
\hline Between Groups & 0.014045 & 1 & 0.014045 & 0.000805 & 0.977672 & 4.413873 \\
\hline Within Groups & 313.90801 & 18 & 17.439334 & & & \\
\hline Total & 313.922055 & 19 & & & & \\
\hline
\end{tabular}




\begin{tabular}{|c|c|c|c|c|c|c|}
\hline \multirow{2}{*}{\multicolumn{7}{|c|}{$\begin{array}{l}\text { SVh-SZ } \\
\text { ANOVA: Single Factor }(p=0.05)\end{array}$}} \\
\hline & & & & & & \\
\hline & & & & & & \\
\hline \multicolumn{7}{|l|}{ SUMMARY } \\
\hline Groups & Count & Sum & Average & Variance & & \\
\hline DWT & 10 & 930 & 93 & 18.50889 & & \\
\hline CDWT & 10 & 915.6 & 91.56 & 20.70044 & & \\
\hline \multicolumn{7}{|l|}{ ANOVA } \\
\hline Source of Variation & $S S$ & $d f$ & MS & $F$ & $P$-value & $F_{\text {crit }}$ \\
\hline Between Groups & 10.368 & 1 & 10.368 & 0.528854 & 0.476442 & 4.413873 \\
\hline Within Groups & 352.884 & 18 & 19.604667 & & & \\
\hline Total & 363.252 & 19 & & & & \\
\hline & & & & & & \\
\hline \multicolumn{7}{|c|}{ DWT-RA } \\
\hline \multicolumn{7}{|c|}{ ANOVA: Single Factor $(p=0.05)$} \\
\hline & & & & & & \\
\hline \multicolumn{7}{|l|}{ SUMMARY } \\
\hline Groups & Count & Sum & Average & Variance & & \\
\hline ANN & 10 & 523.9 & 52.39 & 58.51878 & & \\
\hline SVM & 10 & 714.6 & 71.46 & 39.00656 & & \\
\hline \multicolumn{7}{|l|}{ ANOVA } \\
\hline Source of Variation & $S S$ & $d f$ & MS & $F$ & P-value & $F_{c r i t}$ \\
\hline Between Groups & 1818.3245 & 1 & 1818.3245 & 37.28928 & 9.07E-06 & 4.413873 \\
\hline Within Groups & 877.728 & 18 & 48.762667 & & & \\
\hline Total & 2696.0525 & 19 & & & & \\
\hline & & & & & & \\
\hline \multicolumn{7}{|c|}{ DWT-CP } \\
\hline \multicolumn{7}{|c|}{ ANOVA: Single Factor $(p=0.05)$} \\
\hline & & & & & & \\
\hline \multicolumn{7}{|l|}{ SUMMARY } \\
\hline Groups & Count & Sum & Average & Variance & & \\
\hline ANN & 10 & 893.9 & 89.39 & 54.75211 & & \\
\hline SVMI & 10 & 826.5 & 82.65 & 87.72056 & & \\
\hline \multicolumn{7}{|l|}{ ANOVA } \\
\hline Source of Variation & $S S$ & $d f$ & MS & $F$ & P-value & $F_{\text {crit }}$ \\
\hline Between Groups & 227.138 & 1 & 227.138 & 3.188513 & 0.091017 & 4.413873 \\
\hline Within Groups & 1282.254 & 18 & 71.236333 & & & \\
\hline Total & 1509.392 & 19 & & & & \\
\hline
\end{tabular}




\begin{tabular}{|c|c|c|c|c|c|c|}
\hline \multirow{2}{*}{\multicolumn{7}{|c|}{$\begin{array}{l}\text { DWT-HE } \\
\text { ANOVA: Single Factor }(p=0.05)\end{array}$}} \\
\hline & & & & & & \\
\hline \multirow{2}{*}{\multicolumn{7}{|c|}{ SUMMARY }} \\
\hline & & & & & & \\
\hline Groups & Count & Sum & Average & Variance & & \\
\hline ANN & 10 & 759.8 & 75.98 & 61.99733 & & \\
\hline SVM & 10 & 823.47 & 82.347 & 40.30942 & & \\
\hline \multicolumn{7}{|l|}{ ANOVA } \\
\hline Source of Variation & SS & $d f$ & MS & $F$ & P-value & $F_{\text {crit }}$ \\
\hline Between Groups & 202.693445 & 1 & 202.69345 & 3.962464 & 0.06193 & 4.413873 \\
\hline Within Groups & 920.76081 & 18 & 51.153378 & & & \\
\hline Total & 1123.45426 & 19 & & & & \\
\hline & & & & & & \\
\hline \multicolumn{7}{|c|}{ DWT-So } \\
\hline \multicolumn{7}{|c|}{ ANOVA: Single Factor $(p=0.05)$} \\
\hline \multirow{2}{*}{\multicolumn{7}{|c|}{ SUMMARY }} \\
\hline & & & & & & \\
\hline Groups & Count & Sum & Average & Variance & & \\
\hline ANN & 10 & 776.4 & 77.64 & 30.44711 & & \\
\hline SVM & 10 & 916.17 & 91.617 & 15.88078 & & \\
\hline \multicolumn{7}{|l|}{ ANOVA } \\
\hline Source of Variation & SS & $d f$ & MS & $F$ & P-value & $F_{c r i t}$ \\
\hline Between Groups & 976.782645 & 1 & 976.78265 & 42.16823 & 4.17E-06 & 4.413873 \\
\hline Within Groups & 416.95101 & 18 & 23.163945 & & & \\
\hline Total & 1393.73366 & 19 & & & & \\
\hline & & & & & & \\
\hline \multicolumn{7}{|c|}{ DWT-SZ } \\
\hline \multicolumn{7}{|c|}{ ANOVA: Single Factor $(p=0.05)$} \\
\hline & & & & & & \\
\hline \multicolumn{7}{|l|}{ SUMMARY } \\
\hline Groups & Count & Sum & Average & Variance & & \\
\hline ANN & 10 & 875.7 & 87.57 & 40.54233 & & \\
\hline SVMI & 10 & 930 & 93 & 18.50889 & & \\
\hline \multicolumn{7}{|l|}{ ANOVA } \\
\hline Source of Variation & $S S$ & $d f$ & MS & $F$ & P-value & $F_{\text {crit }}$ \\
\hline Between Groups & 147.4245 & 1 & 147.4245 & 4.993106 & 0.038368 & 4.413873 \\
\hline Within Groups & 531.461 & 18 & 29.525611 & & & \\
\hline Total & 678.8855 & 19 & & & & \\
\hline
\end{tabular}




\begin{tabular}{|c|c|c|c|c|c|c|}
\hline \multirow{2}{*}{\multicolumn{7}{|c|}{$\begin{array}{l}\text { DWT-ME } \\
\text { ANOVA Single Factor }(\mathrm{p}=0,05)\end{array}$}} \\
\hline \\
\hline & & & & & & \\
\hline \multicolumn{7}{|c|}{ SUMMARY } \\
\hline Groups & Count & Sum & Average & Variance & & \\
\hline ANN & 10 & 759.8 & 75.98 & 61.99733 & & \\
\hline SVM & 10 & 823.47 & 82.347 & 40.30942 & & \\
\hline \multicolumn{7}{|l|}{ ANOVA } \\
\hline Source of Variation & $S S$ & $d f$ & MS & $F$ & $P$-value & $F_{\text {crit }}$ \\
\hline Between Groups & 202.693445 & 1 & 202.69345 & 3.962464 & 0.06193 & 4.413873 \\
\hline Within Groups & 920.76081 & 18 & 51.153378 & & & \\
\hline Total & 1123.45426 & 19 & & & & \\
\hline \multirow{2}{*}{\multicolumn{7}{|c|}{ DWT-SO }} \\
\hline & & & & & & \\
\hline \multicolumn{7}{|c|}{ ANOVA: Single Factor $(p=0.05)$} \\
\hline & & & & & & \\
\hline \multicolumn{7}{|l|}{ SUMMARY } \\
\hline Groups & Count & Sum & Average & Variance & & \\
\hline ANN & 10 & 776.4 & 77.64 & 30.44711 & & \\
\hline SVM & 10 & 916.17 & 91.617 & 15.88078 & & \\
\hline \multicolumn{7}{|l|}{ ANOVA } \\
\hline Source of Variation & SS & $d f$ & MS & $F$ & P-value & $F_{\text {crit }}$ \\
\hline Between Groups & 976.782645 & 1 & 976.78265 & 42.16823 & 4.17E-06 & 4.413873 \\
\hline Within Groups & 416.95101 & 18 & 23.163945 & & & \\
\hline Total & 1393.73366 & 19 & & & & \\
\hline & & & & & & \\
\hline \multicolumn{7}{|c|}{ DWT-SZ } \\
\hline \multicolumn{7}{|c|}{ ANOVA: Single Factor $(p=0.05)$} \\
\hline & & & & & & \\
\hline \multicolumn{7}{|l|}{ SUMMARY } \\
\hline Groups & Count & Sum & Average & Variance & & \\
\hline ANN & 10 & 875.7 & 87.57 & 40.54233 & & \\
\hline SVMH & 10 & 930 & 93 & 18.50889 & & \\
\hline \multicolumn{7}{|l|}{ ANOVA } \\
\hline Source of Variation & $S S$ & $d f$ & MS & $F$ & P-value & $F_{\text {crit }}$ \\
\hline Between Groups & 147.4245 & 1 & 147.4245 & 4.993106 & 0.038368 & 4.413873 \\
\hline Within Groups & 531.461 & 18 & 29.525611 & & & \\
\hline Total & 678.8855 & 19 & & & & \\
\hline
\end{tabular}




\begin{tabular}{|c|c|c|c|c|c|c|}
\hline CDWT-SO & & & & & & \\
\hline \multicolumn{7}{|c|}{ ANOVA: Single Factor $(p=0.05)$} \\
\hline & & & & & & \\
\hline \multicolumn{7}{|l|}{ SUMMARY } \\
\hline Groups & Count & Sum & Average & Variance & & \\
\hline ANN & 10 & 850.9 & 85.09 & 20.87211 & & \\
\hline SVM & 10 & 916.7 & 91.67 & 18.99789 & & \\
\hline \multicolumn{7}{|l|}{ ANOVA } \\
\hline Source of Variation & SS & $d f$ & MS & $F$ & $P$-value & $F_{\text {crit }}$ \\
\hline Between Groups & 216.482 & 1 & 216.482 & 10.85939 & 0.004022 & 4.413873 \\
\hline Within Groups & 358.83 & 18 & 19.935 & & & \\
\hline Total & 575.312 & 19 & & & & \\
\hline & & & & & & \\
\hline \multicolumn{7}{|c|}{\begin{tabular}{l|l} 
CDWT-SZ & \\
\end{tabular}} \\
\hline \multicolumn{7}{|c|}{ ANOVA: Single Factor $(p=0.05)$} \\
\hline & & & & & & \\
\hline \multicolumn{7}{|l|}{ SUMMARY } \\
\hline Groups & Count & Sum & Average & Variance & & \\
\hline ANN & 10 & 883.3 & 88.33 & 14.88011 & & \\
\hline SVM & 10 & 915.6 & 91.56 & 20.70044 & & \\
\hline \multicolumn{7}{|l|}{ ANOVA } \\
\hline Source of Variation & SS & $d f$ & MS & $F$ & P-value & $F_{\text {crit }}$ \\
\hline Between Groups & 52.1645 & 1 & 52.1645 & 2.932191 & 0.104003 & 4.413873 \\
\hline Within Groups & 320.225 & 18 & 17.790278 & & & \\
\hline Total & 372.3895 & 19 & & & & \\
\hline
\end{tabular}




\section{Appendix B}

\section{Grid searching results of selecting parameters for SVM models}

B.1 Grid searching for $(C, \gamma)$ for SVM classifiers of Chapter 4

\begin{tabular}{|c|c|c|c|}
\hline Parameters & $C$ & $\gamma$ & Best Accuracy (\%) \\
\hline FFT & 32 & 0.0078125 & 82.28 \\
\hline DWT & 2 & 0.0125 & 87.34 \\
\hline
\end{tabular}



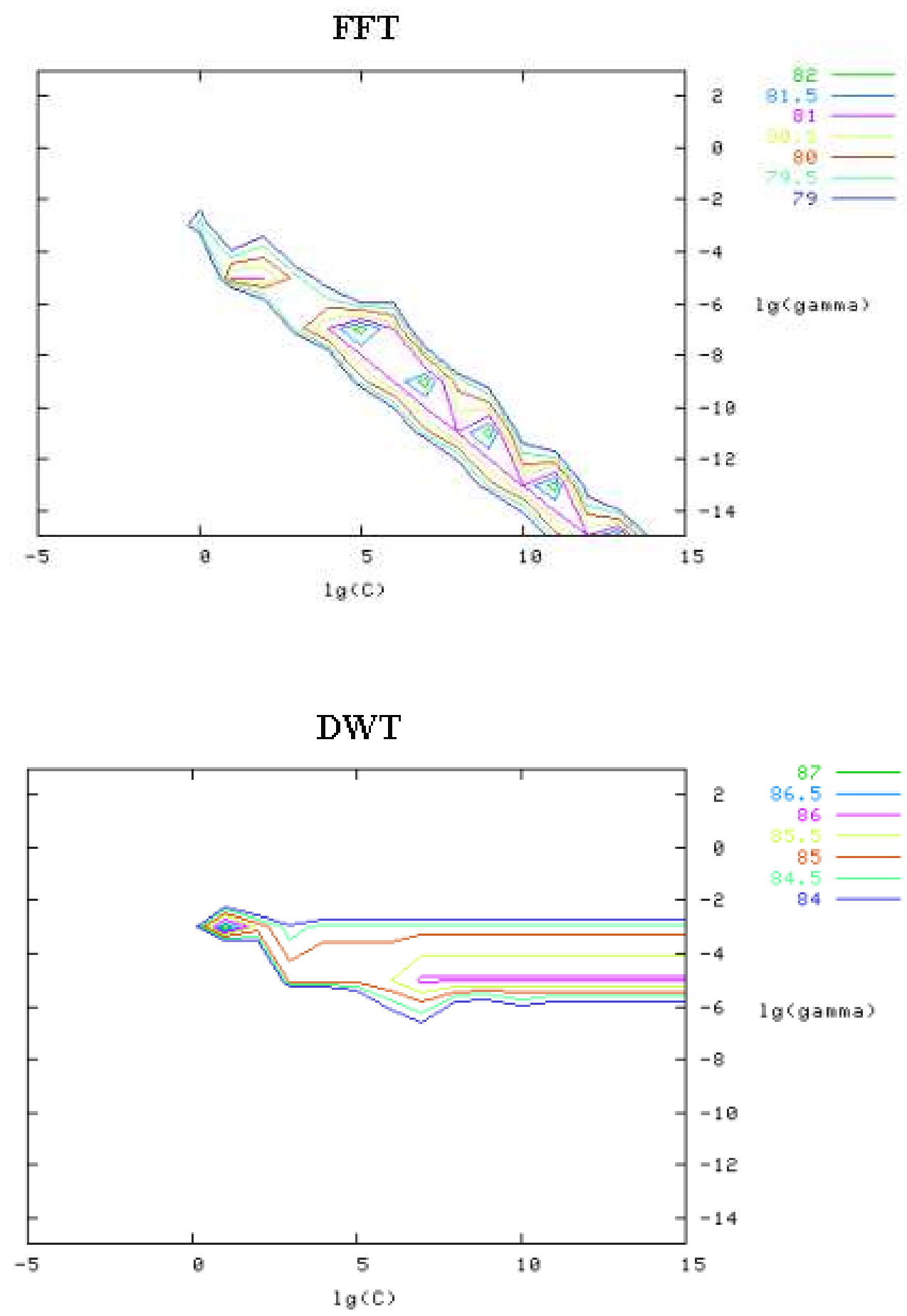
B.2 Grid searching for $(C, \gamma)$ for SVM classifiers of Chapter 5

\begin{tabular}{|c|c|c|c|}
\hline Parameters & $C$ & $\gamma$ & Best Accuracy (\%) \\
\hline Dest Set & & & \\
\hline DWT_RA & 1 & 0.125 & 74.5 \\
\hline DWT_CP & 32 & 0.03125 & 89.4 \\
\hline DWT_SO & 1 & 0.03125 & 82 \\
\hline DWT_SZ & 8 & 0.125 & 91 \\
\hline CDWT_RA & 8 & 0.03125 & 93.5 \\
\hline CDWT_CP & 128 & 0.0078125 & 81.01 \\
\hline CDWT_ME & 32 & 0.00049 & 82.54 \\
\hline CDWT_SO & 32 & 0.125 & 89.87 \\
\hline CDWT_SZ & 1 & 0.0078125 & 93.5 \\
\hline
\end{tabular}



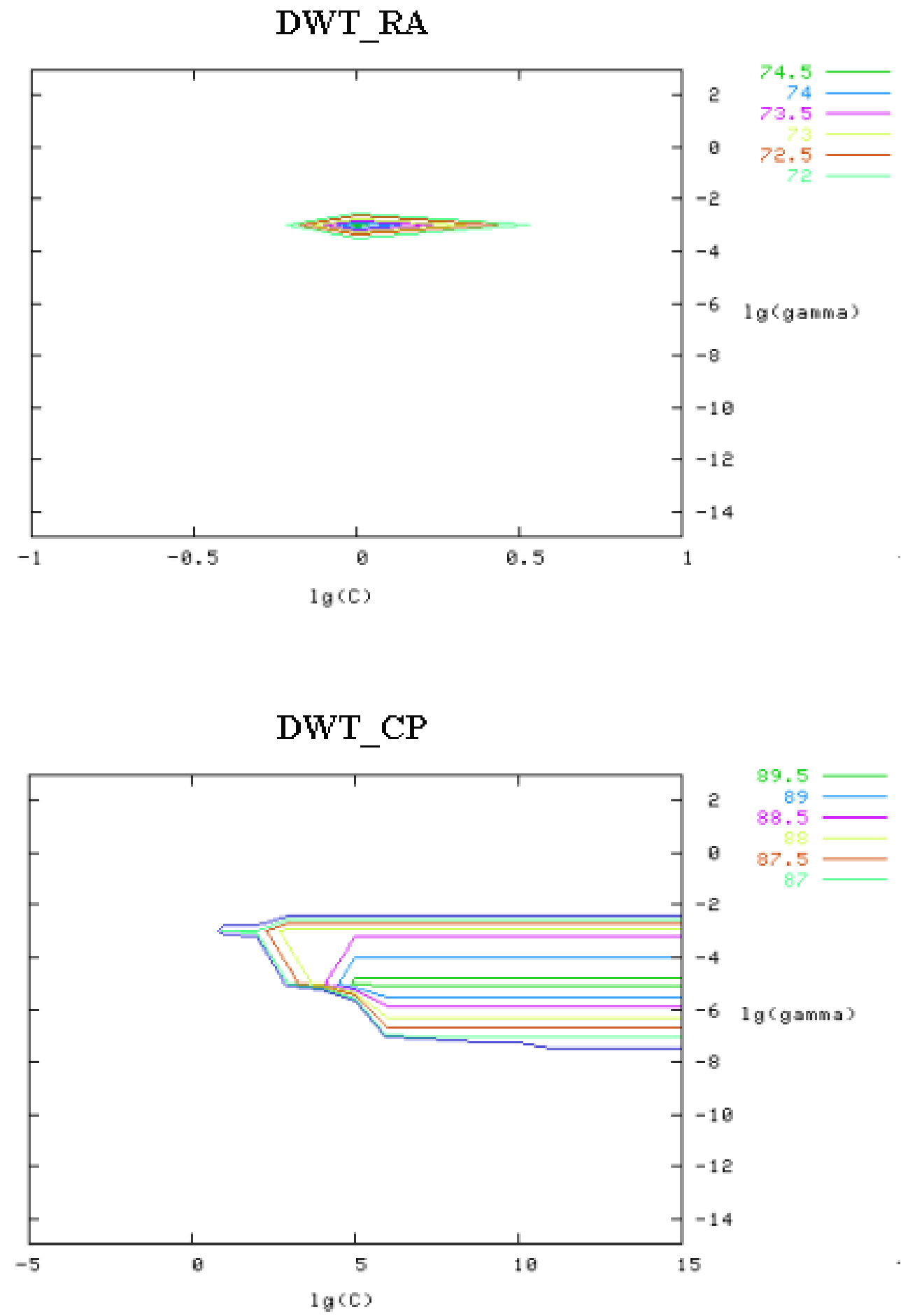

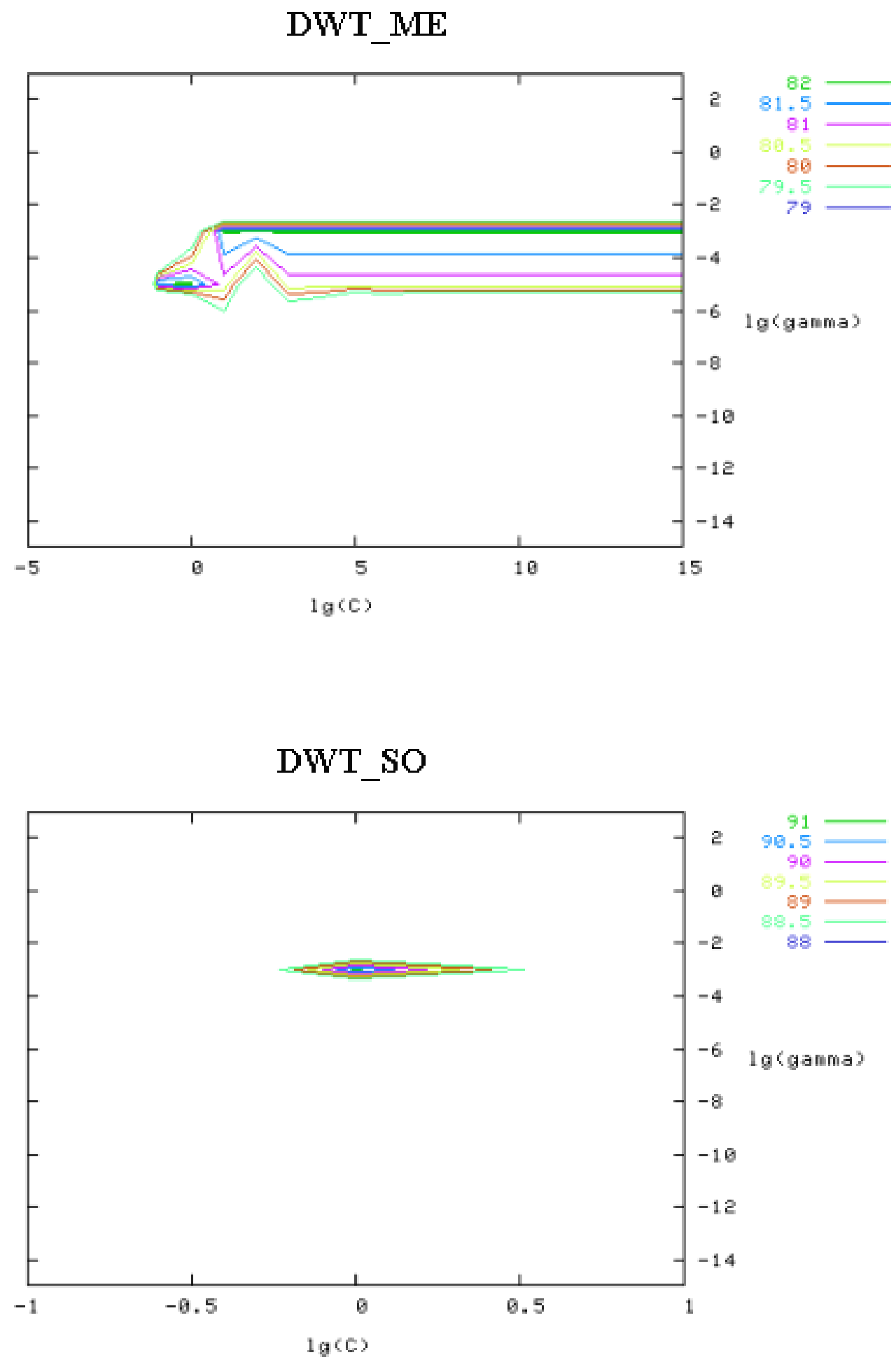

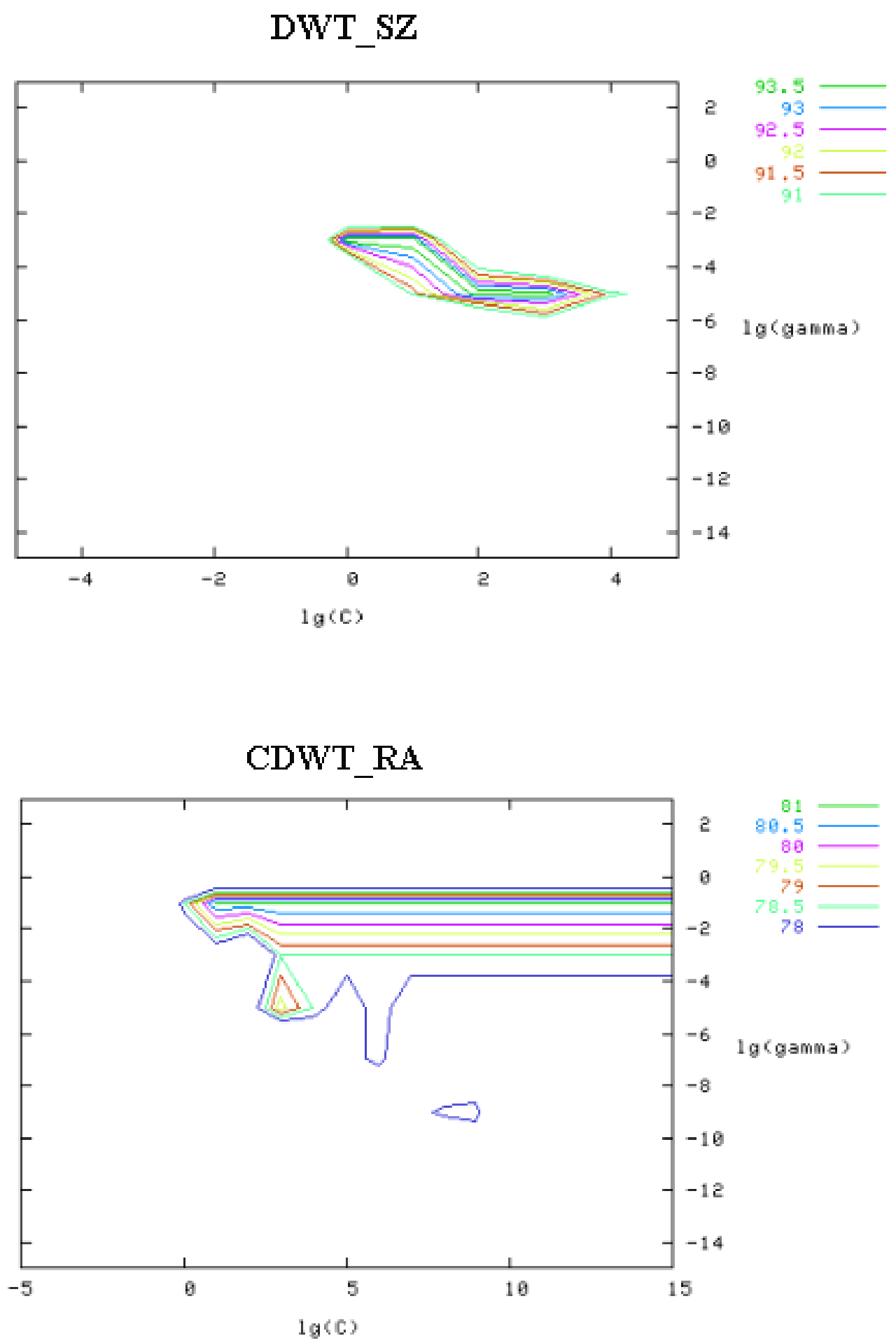

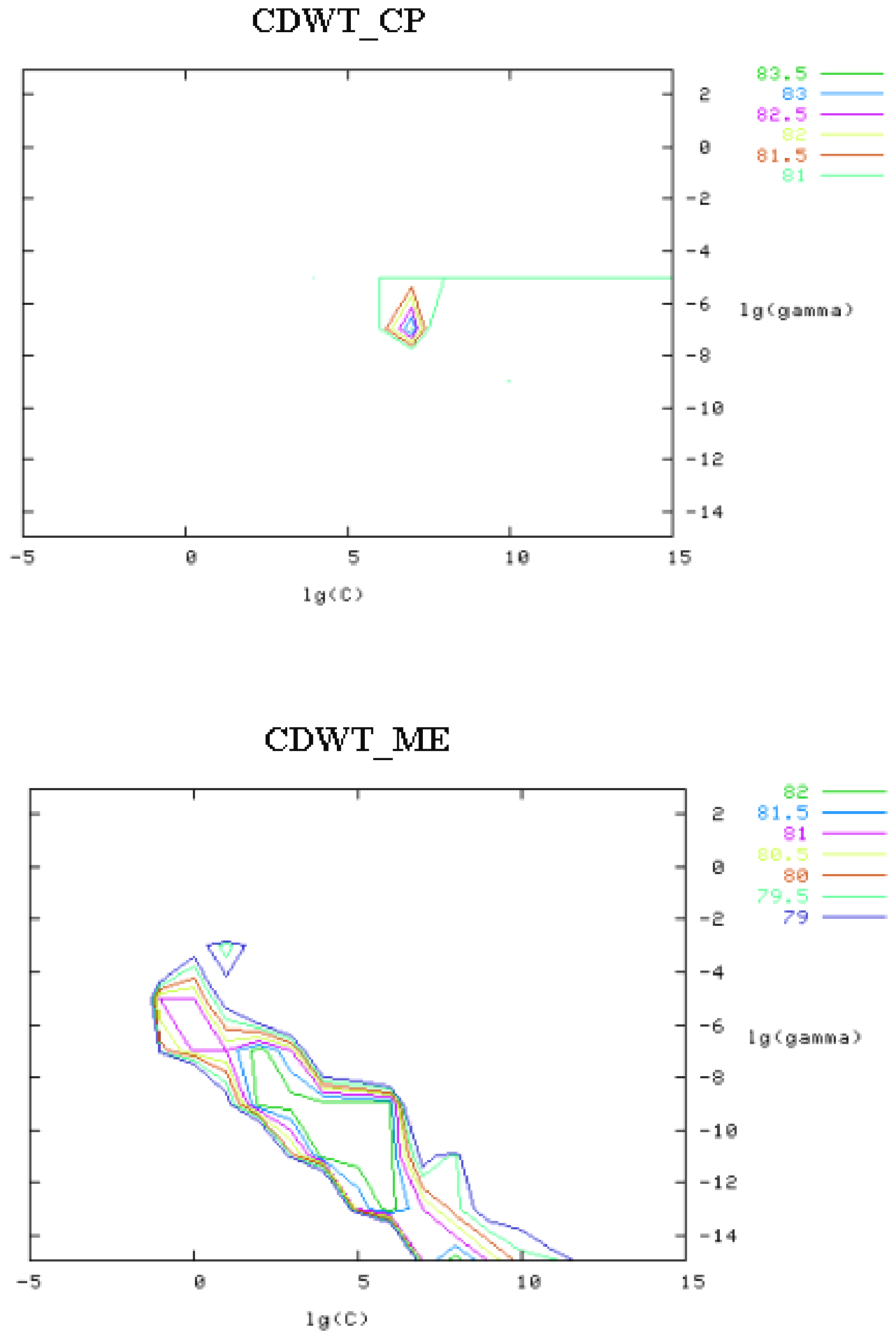


\section{CDWT_SO}

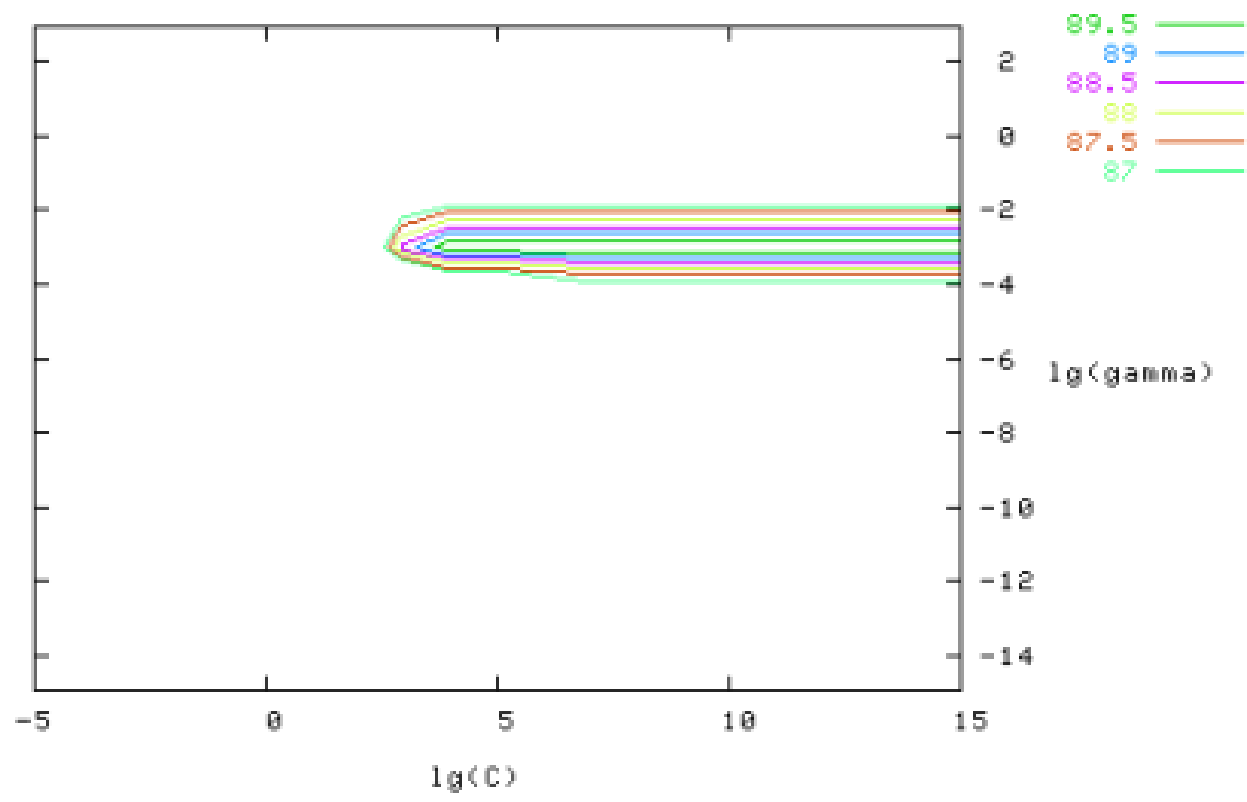

CDWT_SZ

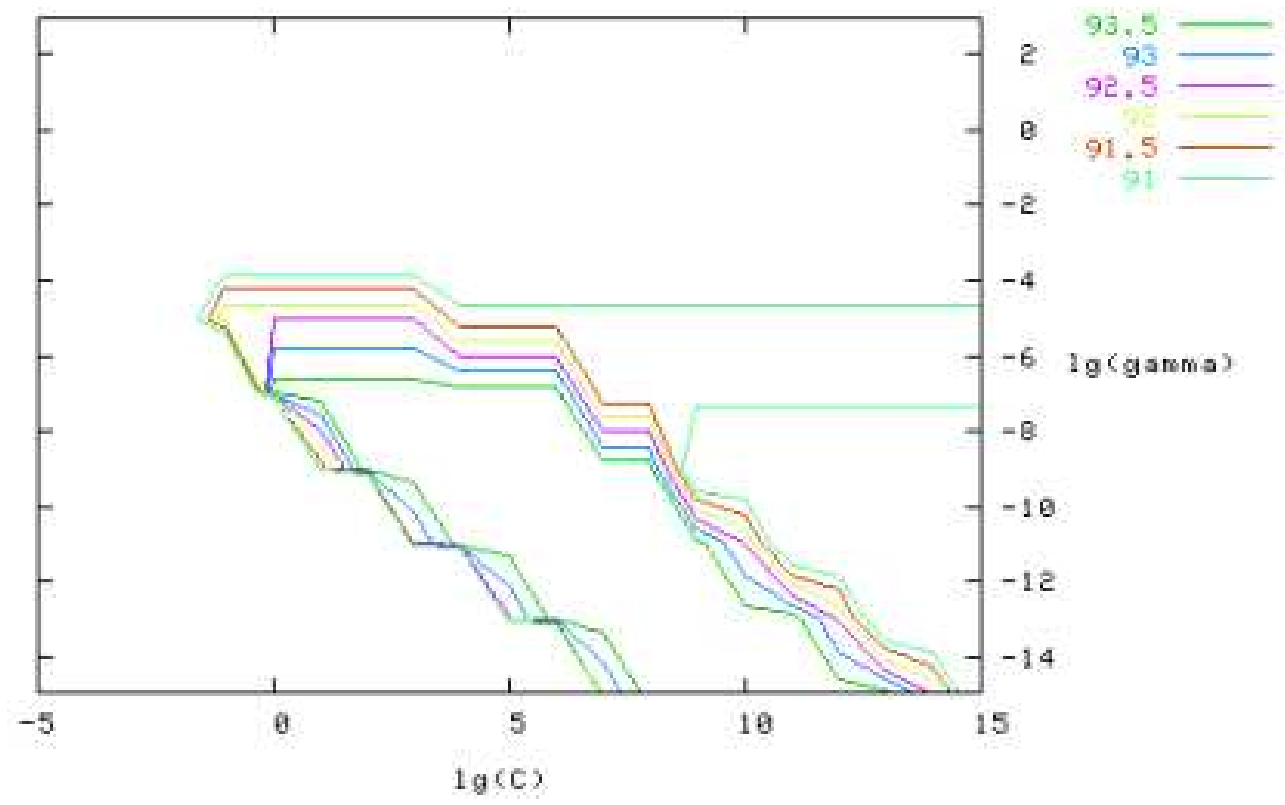




\section{Appendix C}

\section{Methods for Combining Classifiers}

There are various combination methods that can be applied when each classifier (also called an expert) $e$ outputs a unique label or class for each input pattern. Among those combination methods, we applied three combination methods for the experiment presented in Chapter 6 of this thesis. They are Bayesian Combination (BC), Distribution Summation (DS) and Likelihood Combination (LC).

\section{C.1 Bayesian Combination}

This method applies the Bayesian decision rule in order to determine a weight and assign it to the decision made by each individual classifier. Through this method, different weights are applied to patterns depending on the decision made by the expert taking into consideration the performance of each expert on the training samples of each class. The performance of each classifier is computed through its confusion matrix $C$ on a training set of data. For a problem with $M$ possible classes plus the reject option, $C$ is an $M \times(M+1)$ matrix in which the entry $C_{i j}$ denotes the number of patterns with actual class $i$ that is assigned class $j$ by the classifier when $j \leq M$, and when $j=M+1$, it represents the number of patterns that are rejected.

From the Matrix $C$, we can obtain the total number of samples belonging to class $i$ as the 
row sum $\Sigma_{j=1}^{M+1} C_{i j}$, while the column sum $\Sigma_{i=1}^{M} C_{i j}$ represents the total number of samples that are assigned class $j$ by this expert. When there are $K$ experts, there would be $K$ confusion matrices $C^{(k)}, 1 \leq k \leq K$. Consequently, the conditional probability that a pattern $x$ actually belongs to class $i$, given that expert $k$ assigns it to class $j$, can be estimated as

$$
P\left(x \in C_{i} \mid e_{k}(x)=j\right)=\frac{C_{i j}^{(k)}}{\sum_{i=1}^{M} C_{i j}^{(k)}},
$$

and this term can represent the degree of accuracy when expert $k$ assigns class $i$ to a sample.

For any pattern $x$ such that the classification results by the $K$ experts are $e_{k}(x)=j_{k}$ for $1 \leq k \leq K$, we can define a belief value that $x$ belongs to class $i$ as

$$
\operatorname{bel}(i)=P\left(x \in C_{i} \mid e_{1}(x)=j_{1}, \cdots, e_{K}(x)=j_{k}\right) .
$$

By applying the Bayes' formula and assuming independence of the expert decisions, bel $(i)$ can be approximated by

$$
\operatorname{bel}(i)=\frac{\prod_{k=1}^{K} P\left(x \in C_{i} \mid e_{k}(x)=j_{k}\right)}{\sum_{i=1}^{M} \prod_{k=1}^{K} P\left(x \in C_{i} \mid e_{k}(x)=j_{k}\right)}
$$

for $1 \leq i \leq M$.

For any input pattern $x$, we can assign $x$ to class $j$ if $\operatorname{bel}(j)>\operatorname{bel}(i)$ for all $i \neq j$ and $\operatorname{bel}(j)>\alpha$ for a threshold $\alpha$. Otherwise $x$ is rejected, and it is also rejected if $e_{K}(x)=M+1$ for all $k$ (i.e. if $x$ is rejected by all classifiers). The results obtained from this method depend on the value of $\alpha$ chosen. As $\alpha$ increases, so does the degree of certainty expected of the decision; therefore the error rate would decrease, but the recognition rate would be also lower. 


\section{C.2 Distribution Summation}

This method associates a $k$-component vector (distribution) with each individual classifier, where $k$ denotes the number of classes. The vector consists of the numbers of training examples from all $k$ classes covered by that classifier. A component-wise sum is formed over all classifiers (of all classes) to produce a combined vector. In order to make a combined decision, the highest number in the summed vector is sought and this method will predict the class corresponding to the element of highest value.

For the classification problem of the experiment in Chapter 6, each individual classifier keeps a 2-component vector for each class $(\mathrm{MC}$ and $\mathrm{CR})$ and they are denoted by $\left(C 1_{M C}, C 1_{C R}\right)$ for the MC class and $\left(C 2_{M C}, C 2_{C R}\right)$ for the CR class. $\left(C 1_{M C}, C 1_{C R}\right)$ indicates that the classifier's decision (MC is the class predicted) is true for $C 1_{M C}$ training examples of class $\mathrm{MC}$ and for $C 1_{C R}$ training examples of class CR. In a pairwise way, $\left(C 2_{M C}, C 2_{C R}\right)$ indicates that the classifier's decision (CR is the class predicted) is true for $C 2_{M C}$ training examples of class $\mathrm{MC}$ and for $C 2_{C R}$ training examples of class $\mathrm{CR}$. When the predictions by multiple models are combined, all of the vectors corresponding to the class each classifier predicts are combined using vectorial summation. For example, one classifier predicts MC for a sample and the other predicts $\mathrm{CR}$ for the same sample, so the first component vector of the first classifier and the second vector of the second classifier are summed and the summed elements are compared. If the first element of the summed vector is larger than its second element, $\mathrm{MC}$ is the combined decision and vice versa.

\section{C.3 Likelihood Combination}

This method associates "the degree of logical sufficiency of the rule" (LS) [30] with each rule. In the context of classification, the LS of a rule of Class $i$ is defined as the ratio of the following probabilities

$$
\frac{p\left(\operatorname{rule}(\tau)=\text { true } \mid \tau \in \text { Class }_{i}\right)}{p\left(\operatorname{rule}(\tau)=\text { true } \mid \tau \notin \text { Class }_{i}\right)}
$$


where $\tau$ is a random example. Each probability is estimated using the Laplace estimate [51]. This method uses the odds form of Bayes rule [30] which is also called the naive Bayes combination [53]. This rule can be restated for our purposes as

$$
O\left(\text { Class }_{i} \mid M_{i 1}\right)=\frac{O\left(\text { Class }_{i}\right) \times O\left(M_{i 1} \mid \text { Class }_{i}\right)}{O\left(M_{i 1}\right)} \propto O\left(\text { Class }_{i}\right) \times O\left(M_{i 1} \mid \text { Class }_{i}\right)
$$

where $M_{i 1}$ denotes the description for class $i$ in the first individual model. The odds of a proposition with probability $p$ are defined to be $p /(1-p)$.

In extending this method to multiple models, the prior odds of the class only appear once: let $M_{i}$ denote the set of class descriptions for class $i$ and $M_{i j}$ denote one such class description. Then the posterior odds of Class $_{i}$ are given by:

$$
O\left(\text { Class }_{i} \mid M_{i}\right) \propto O\left(\text { Class }_{i}\right) \times \prod_{j} O\left(\text { Class }_{i} \mid M_{i j}\right)
$$

For the term $O\left(\right.$ Class $\left._{i} \mid M_{i j}\right)$ we use the LS of the most reliable satisfied rule in $M_{i j}$. 


\section{Appendix D}

\section{The result weights for three combining methods}

\begin{tabular}{|c|c|c|c|c|c|c|c|c|c|c|c|c|c|}
\hline \multicolumn{14}{|c|}{ Cross-validation test 1} \\
\hline \multirow{2}{*}{ Class } & \multirow{2}{*}{$\begin{array}{l}\text { Combining } \\
\text { Methods }\end{array}$} & \multicolumn{6}{|c|}{ FFT } & \multicolumn{6}{|c|}{ DWT-CP } \\
\hline & & SVM & ANNN1 & ANN2 & ANN3 & ANN4 & ANNS & SVM & ANN1 & ANN2 & ANN3 & ANN4 & NNE \\
\hline \multirow{3}{*}{ MC } & $\mathrm{BC}$ & 0.89 & 1.0 & 0.86 & 1.0 & 0.83 & 1.0 & 0.9 & 0.7 & 0.6 & 0.73 & 0.62 & 1.82 \\
\hline & & 1) & $(8,0)$ & $(6,1)$ & $(7,0)$ & $(5,1)$ & $(4,0)$ & & $(7,3)$ & $(6,4)$ & $(8,3)$ & $(8,5)$ & \\
\hline & & 22 & 1.0 & 4.67 & 1.0 & 3.89 & 1.0 & 7.0 & 1.82 & 1.17 & 2.07 & 1.24 & 3.5 \\
\hline \multirow{3}{*}{$\mathrm{CR}$} & & 36 & 0.88 & 0.67 & 0.78 & 0.6 & 0.58 & 1.0 & 0.67 & 0.5 & 0.8 & 0.67 & 1.0 \\
\hline & DS & 6) & $(1,7)$ & $(3,6)$ & $(2,7)$ & $(4,6)$ & $(5,7)$ & $(0,6)$ & $(2,4)$ & $(3,3)$ & $(1,4)$ & $(1,2)$ & $(0,5)$ \\
\hline & & & 9 & 2.57 & 4.5 & 1.93 & 1.8 & & 2.57 & 1.29 & 5.14 & 2.57 & 1.0 \\
\hline \multirow[b]{2}{*}{ Class } & Com & \multicolumn{6}{|c|}{ DWT ME } & \multicolumn{6}{|c|}{ DWT-RA } \\
\hline & Method & SVM & ANN1 1 & ANN2 & ANNN3 & A.NN4 & ANNS & SVM & ANN1 & ANN2 & ANN3 & ANN4 & ANNE \\
\hline \multirow{3}{*}{ MC } & $\mathrm{BC}$ & 0.89 & 1.0 & 0.89 & 1.0 & 1.0 & 1.0 & 0.67 & 0.64 & 0.73 & 0.69 & 0.69 & 0.69 \\
\hline & $\mathrm{D}$ & 1) & $(6,0)$ & $(8,1)$ & $(9,0)$ & $(8,0)$ & $(9,0)$ & $(8,4)$ & $(9,5)$ & $(8,3)$ & $(9,4)$ & $(9,4)$ & $(9,4)$ \\
\hline & & 22 & 1.0 & 6.22 & 0 & 1.0 & 0 & 1.56 & 1.4 & 2.07 & 1.75 & 1.75 & 1.75 \\
\hline \multirow{3}{*}{$\mathrm{CR}$} & & 86 & 0.7 & 0.86 & 1.0 & 0.88 & 1.0 & 0.75 & 1.0 & 0.8 & 1.0 & 1.0 & 1.0 \\
\hline & DS & $(1,6)$ & $(3,7)$ & $(1,6)$ & $(0,7)$ & $(1,7)$ & $(0,7)$ & (1.3) & $(0.2)$ & $(1.4)$ & (0.3) & $(0.3)$ & $(0.3)$ \\
\hline & & 7.71 & 3.0 & 7.71 & 1.0 & 0.88 & 1.0 & 3.86 & 1.0 & 5.14 & 1.0 & 1.0 & 1.0 \\
\hline \multirow{2}{*}{ Class } & \multirow{2}{*}{\multicolumn{5}{|c|}{ DWT-SO }} & & & \\
\hline & & & & ANN2 & ANNN3 & & & \multicolumn{6}{|c|}{\begin{tabular}{|c|c|c|c|}
\multicolumn{2}{c}{ DWT-SZ } \\
SVM $\mid$ ANN1 ANN2|ANN3
\end{tabular}} \\
\hline \multirow{3}{*}{ MC } & & 0.82 & 0.8 & 0.67 & 0.88 & 0.7 & 0.9 & 1.0 & 0.9 & 0.9 & 1.0 & 1.0 & 1.0 \\
\hline & DS & $(9,2)$ & $(8,2)$ & $(4,2)$ & $(7,1)$ & $(7,3)$ & $(9,1)$ & $(9,1)$ & $(9,1)$ & $(8,0)$ & $(8,0)$ & $(8,0)$ & $(9,0)$ \\
\hline & & 3.5 & 3.11 & 1.56 & 5.44 & 1.81 & 7.0 & 0 & 7.0 & 7.0 & 1.0 & 1.0 & 0 \\
\hline \multirow{3}{*}{$\mathrm{CR}$} & & 1.0 & 0.83 & 0.5 & 0.75 & 0.67 & 1.0 & 1.0 & 1.0 & 1.0 & 0.88 & 0.88 & 0.58 \\
\hline & $\overline{D S}$ & $(0,5)$ & $(1,5)$ & $(5,5)$ & $(2,6)$ & $(2,4)$ & $(0,6)$ & $(0,7)$ & $(0,6)$ & $(0,6)$ & $(1,7)$ & $(1,7)$ & $(0,7)$ \\
\hline & & $\frac{x}{10}$ & 6.43 & 1.29 & 3.86 & 2.57 & 1.0 & 1.0 & 1.0 & 1.0 & 9.0 & 9.0 & 1.0 \\
\hline
\end{tabular}




\begin{tabular}{|c|c|c|c|c|c|c|c|c|c|c|c|c|c|}
\hline \multicolumn{14}{|c|}{ Cross-validation test 2} \\
\hline \multirow{2}{*}{ Class } & \multirow{2}{*}{$\begin{array}{l}\text { Combining } \\
\text { Methods }\end{array}$} & \multicolumn{6}{|c|}{ FFT } & \multicolumn{6}{|c|}{ DWT-CP } \\
\hline & & SVM & ANN1 & ANN2 & ANN3 & ANN4 & ANNS & SVM & ANN1 & ANN2 & ANN3 & ANN4 & NN5 \\
\hline \multirow{3}{*}{ MC } & $\mathrm{BC}$ & 0.91 & 0.88 & \begin{tabular}{|l|}
0.88 \\
\end{tabular} & 0.86 & 0.9 & 0.86 & \begin{tabular}{|l|}
0.88 \\
\end{tabular} & 0.8 & 0.8 & 0.8 & 1.0 & .78 \\
\hline & $\mathrm{DS}$ & $(10,1)$ & $(7,1)$ & $(7,1)$ & $(6,1)$ & $(9,1)$ & $(6,1)$ & $(7,1)$ & $(8,2)$ & $(8,2)$ & $(8,2)$ & $(7,0)$ & $(7,2)$ \\
\hline & & & 3.18 & 3.18 & 2.73 & 4.09 & 2.73 & 3.18 & 1.82 & 1.82 & 1.82 & 1.0 & 1.60 \\
\hline \multirow{3}{*}{$\mathrm{CR}$} & & 8 & 0.5 & 0.5 & 0.44 & 0.67 & 0.44 & 0.5 & 0.5 & 0.5 & 0.5 & 0.56 & 1.43 \\
\hline & & $(1,4)$ & $(4,4)$ & $(4,4)$ & $(5,4)$ & $(2,4)$ & $(5,4)$ & $(4,4)$ & $(3,3)$ & $(3,3)$ & $(3,3)$ & $(4,5)$ & $(4,3)$ \\
\hline & & 8.8 & 2.2 & 2.2 & 1.76 & 4.4 & 1.76 & 2.2 & 2.2 & 2.2 & 2.2 & 2.75 & 1.65 \\
\hline \multirow{2}{*}{ Class } & \multirow{2}{*}{\multicolumn{7}{|c|}{ 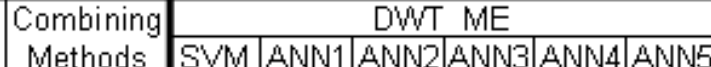 }} & \multicolumn{6}{|c|}{ DWT-RA } \\
\hline & & & & & & & & \multicolumn{6}{|c|}{\begin{tabular}{|l|l|} 
ANN2 & ANN3
\end{tabular}} \\
\hline \multirow{3}{*}{$M C$} & & 0.92 & 0.92 & 0.91 & 1.0 & 1.0 & 1.0 & 0.9 & 0.71 & 0.71 & 0.67 & 0.86 & 0.63 \\
\hline & & $(11,1)$ & $(11,1)$ & $(10,1)$ & $(9,0)$ & $(10,0)$ & $(9,0)$ & $(9,1)$ & $(5,2)$ & $(5,2)$ & $(6,3)$ & $(6,1)$ & $(5,3)$ \\
\hline & & 5.0 & 5.0 & 4.55 & 1.0 & 1.0 & 1.0 & 4.09 & 1.14 & 1.14 & 0.91 & 2.73 & 0.76 \\
\hline \multirow{3}{*}{$\mathrm{CR}$} & & & 0.5 & 0.5 & 0.44 & 0.67 & 0.44 & 0.63 & 1.0 & 0.8 & 0.71 & 0.83 & 0.71 \\
\hline & & 15) & $(0,4)$ & $(1,4)$ & $(2,5)$ & $(1,5)$ & $(2,5)$ & $(2,4)$ & $(6,3)$ & $(6,3)$ & $(5,2)$ & $(5,4)$ & $(6,2)$ \\
\hline & & & 1.0 & 8.8 & 5.5 & 11.0 & 5.5 & 4.4 & 1.1 & 1.1 & 0.88 & 1.76 & 0.73 \\
\hline \multirow[b]{2}{*}{ Class } & Combi & \multicolumn{6}{|c|}{ DWT-SO } & \multicolumn{6}{|c|}{ DWN-SZ } \\
\hline & & SVM & ANN1 & ANN2 & ANN3 & ANNA & ANN5 & SVM & ANN1 & ANN2 & ANN3 & ANN4 & ANNE \\
\hline \multirow{3}{*}{$\mathrm{MC}$} & & 0.92 & 0.88 & 0.83 & 0.88 & 1.0 & 0.75 & 1.0 & 0.92 & 0.91 & 1.0 & 1.0 & 1.0 \\
\hline & & $(11,1)$ & $(7,1)$ & $(5,1)$ & $(7,1)$ & $(7,0)$ & $(6,2)$ & $(8,0)$ & $(11,1)$ & $(10,1)$ & $(9,0)$ & $(10.0)$ & $(9,0)$ \\
\hline & & 6.0 & 3.18 & 2.27 & 3.18 & 1.0 & 1.37 & 1.0 & 5.0 & 4.55 & 1.0 & 1.0 & 1.0 \\
\hline \multirow{3}{*}{$C R$} & & 1.0 & 0.5 & 0.4 & 0.5 & 0.56 & 0.38 & 0.63 & 1.0 & 0.8 & 0.71 & 0.83 & 0.71 \\
\hline & $\mathrm{DS}$ & (0.5) & (4.4) & $(6.4)$ & $(4,4)$ & (4.5) & $(5,3)$ & $(3,5)$ & $(0,4)$ & $(14)$ & (25) & $(15)$ & $(25)$ \\
\hline & & 1.0 & 2.2 & 1.47 & 2.2 & 2.75 & 1.32 & 3.67 & 1.0 & 8.8 & 5.5 & 11.0 & 5.5 \\
\hline
\end{tabular}

\begin{tabular}{|c|c|c|c|c|c|c|c|c|c|c|c|c|c|}
\hline \multicolumn{14}{|c|}{ Cross-validation test 3} \\
\hline \multirow{2}{*}{ Class } & \multirow{2}{*}{$\begin{array}{l}\text { Combining } \\
\text { Methods }\end{array}$} & \multicolumn{6}{|c|}{ FFT } & \multicolumn{6}{|c|}{ DWT-CP } \\
\hline & & SVM & ANN1 & ANN2 & ANN3 & ANN4 & ANN5 & SVM & ANN1 & ANN2 & ANN3 & ANNA & ANN5 \\
\hline \multirow{3}{*}{$\mathrm{MC}$} & $\mathrm{BC}$ & $\begin{array}{l}0.67 \\
\end{array}$ & 0.83 & 0.71 & 0.86 & 0.83 & 0.75 & 1.0 & 0.83 & 0.67 & 0.83 & 0.8 & 0.83 \\
\hline & & $(6,3)$ & $(5,1)$ & $(5,2)$ & (6.1) & $(5,1)$ & $(6,2)$ & $(6,0)$ & (5.1) & $(4,2)$ & $(5,1)$ & $(4,1)$ & $(5,1)$ \\
\hline & LC & 2.57 & 6.43 & 3.21 & 7.71 & 6.43 & 3.86 & 1.0 & 6.43 & 2.57 & 6.43 & 5.14 & 6.43 \\
\hline \multirow{3}{*}{$\mathrm{CR}$} & & 86 & 0.8 & 0.78 & 0.89 & 0.8 & 0.88 & 0.9 & 0.8 & 0.7 & 0.8 & 0.83 & 0.8 \\
\hline & & 6) & $(2,8)$ & $(2,7)$ & $(1,8)$ & $(2,8)$ & $(1,7)$ & $(1,9)$ & $(2,8)$ & $(3,7)$ & $(2,8)$ & $(3,8)$ & $(2,8)$ \\
\hline & & & 3.11 & 2.72 & 6.22 & 3.11 & 5.44 & 7.0 & 3.11 & 1.81 & 3.11 & 2.07 & 3.11 \\
\hline \multirow{2}{*}{ Class } & Combi & \multicolumn{6}{|c|}{ DWT ME } & \multicolumn{6}{|c|}{ DWT-RA } \\
\hline & Methods & SVM & ANN1 & ANN2 & ANN3 & ANNA & ANN5 & SVM & ANNN1 & ANNN2 & ANN 3 & ANN4 & ANN5 \\
\hline \multirow{3}{*}{ MC } & $\mathrm{BC}$ & 0.67 & 1.0 & 1.0 & 1.0 & 0.83 & \begin{tabular}{|l|}
1.0 \\
\end{tabular} & 0.71 & 0.67 & 0.6 & 0.67 & 0.67 & 0.63 \\
\hline & & (6.3) & (5,0) & $(6,0)$ & $(5,0)$ & (5.1) & (5,0) & $(5,2)$ & $(4,2)$ & $(3,2)$ & $(4,2)$ & $(4,2)$ & $(5,3)$ \\
\hline & & 1.57 & 1.0 & 1.0 & 1.0 & 6.43 & 1.0 & 3.21 & 2.57 & 1.93 & 2.57 & 2.57 & 2.14 \\
\hline \multirow{3}{*}{$\mathrm{CR}$} & & 0.86 & 0.83 & 0.9 & 0.82 & 0.8 & 0.82 & 0.78 & 0.7 & 0.64 & 0.7 & 0.7 & 0.75 \\
\hline & DS & $(1,6)$ & $(2,9)$ & $(1,9)$ & $(2,9)$ & $(2,8)$ & $(2,9)$ & $(2,7)$ & $(3,7)$ & $(4,7)$ & $(3,7)$ & (37) & $(2,6)$ \\
\hline & $\mathrm{LC}$ & 4.67 & 3.5 & 7.0 & 3.5 & 3.11 & 3.5 & 2.72 & 1.82 & 1.36 & 1.81 & 1.81 & 2.33 \\
\hline \multirow[b]{2}{*}{ Class } & Combin & & \\
\hline & & \multicolumn{6}{|c|}{ DWT-SO } & \multicolumn{6}{|c|}{$\begin{array}{c}\text { DWT-SZ } \\
\text { N1/AN2 ANN3 }\end{array}$} \\
\hline \multirow{3}{*}{$\mathrm{MC}$} & & 0.88 & 0.67 & 0.6 & 0.5 & 0.67 & 0.75 & 1.0 & 1.0 & 1.0 & 1.0 & 0.83 & 0.83 \\
\hline & & $(7,1)$ & $(2,1)$ & $(3,2)$ & $(2,2)$ & $(2,1)$ & $(3,1)$ & $(5,0)$ & $(5,0)$ & $(5,0)$ & $(5,0)$ & $(5,1)$ & $(5,1)$ \\
\hline & & 9.0 & 2.57 & 1.93 & 1.29 & 2.57 & 3.86 & 1.0 & 1.0 & 1.0 & 1.0 & 6.43 & 6.43 \\
\hline \multirow{3}{*}{$\mathrm{CR}$} & & 1.0 & 0.62 & 0.64 & 0.58 & 0.62 & 0.67 & 0.82 & 0.82 & 0.82 & 0.82 & 0.8 & 0.8 \\
\hline & DS & $(0,8)$ & $(5,8)$ & $(4,7)$ & $(5,7)$ & $(5,8)$ & $(4,8)$ & $(2,9)$ & $(2,9)$ & $(2,9)$ & $(2,8)$ & $(2,8)$ & $(2,8)$ \\
\hline & & 1.0 & 1.24 & 1.36 & 1.09 & 1.24 & 1.56 & 3.5 & 3.5 & 3.5 & 3.5 & 3.11 & 3.11 \\
\hline
\end{tabular}




\begin{tabular}{|c|c|c|c|c|c|c|c|c|c|c|c|c|c|}
\hline \multicolumn{14}{|c|}{ Cross-validation test 4} \\
\hline \multirow{2}{*}{ Class } & \multirow{2}{*}{$\begin{array}{l}\text { Combining } \\
\text { Methods }\end{array}$} & \multicolumn{6}{|c|}{ FFT } & \multicolumn{6}{|c|}{ DWT-CP } \\
\hline & & SVM & ANN1 & ANN2 & ANN3 & ANN4 & ANNS & SVM & ANN1 & ANN2 & ANN3 & ANN4 & NN5 \\
\hline \multirow{3}{*}{ MC } & $\mathrm{BC}$ & 0.64 & 0.73 & 0.7 & 0.69 & 0.75 & 0.7 & \begin{tabular}{|l|}
0.82 \\
\end{tabular} & 0.9 & 0.73 & 0.8 & 0.75 & .82 \\
\hline & DS & $(9,5)$ & $(8,3)$ & $(7,3)$ & $(9,4)$ & $(9,3)$ & $(7,3)$ & $(9,2)$ & $(9,1)$ & $(8,3)$ & $(8,2)$ & $(9,3)$ & $(9,2)$ \\
\hline & & 1.4 & 2.07 & 1.81 & 1.75 & 2.33 & 1.81 & 3.5 & 7.0 & 2.07 & 3.11 & 2.33 & 3.5 \\
\hline \multirow{3}{*}{$C R$} & & & 0.8 & 0.67 & 1.0 & 1.0 & 0.67 & 1.0 & 1.0 & 0.8 & 0.83 & 1.0 & 0 \\
\hline & & $(0,2)$ & $(1,4)$ & $(2,4)$ & $(0,3)$ & $(0,4)$ & $(2,4)$ & $(0,5)$ & $(0,6)$ & $(1,4)$ & $(1,5)$ & $(0,4)$ & $(0,5)$ \\
\hline & & 1.0 & 5.14 & 2.57 & 1.0 & 1.0 & 2.57 & 1.0 & 1.0 & 5.14 & 6.43 & 1.0 & 1.0 \\
\hline \multirow{2}{*}{ Class } & \multirow{2}{*}{\multicolumn{7}{|c|}{ 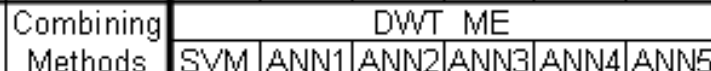 }} & \multicolumn{6}{|c|}{ DWT-RA } \\
\hline & & & & & & & & \multicolumn{6}{|c|}{\begin{tabular}{|l|l|l|l|l|l|} 
ANN1 ANN2 & ANN3 \\
\end{tabular}} \\
\hline \multirow{3}{*}{ MC } & & 0.73 & 0.75 & 0.7 & 0.7 & 0.7 & 0.7 & 0.67 & 0.75 & 0.71 & 0.6 & 0.67 & 0.63 \\
\hline & & $(8,3)$ & $(6,2)$ & $(7,3)$ & $(7,3)$ & $(7,3)$ & $(7,3)$ & $(8,4)$ & $(6,2)$ & $(5,2)$ & $(6.4)$ & (6.3) & $(5,3)$ \\
\hline & & 2.07 & 2.33 & 1.81 & 1.81 & 1.81 & 1.81 & 1.56 & 2.33 & 1.94 & 1.17 & 1.56 & 1.3 \\
\hline \multirow{3}{*}{$\mathrm{CR}$} & & 0.8 & 0.63 & 0.67 & 0.67 & 0.67 & 0.67 & 0.75 & 0.63 & 0.56 & 0.5 & 0.57 & 0.5 \\
\hline & & 4) & $(3,5)$ & $(2,4)$ & $(2,4)$ & $(2,4)$ & $(2,4)$ & $(1,3)$ & $(3,5)$ & $(4,5)$ & $(3,3)$ & $(3,4)$ & $(4,4)$ \\
\hline & & 14 & 2.14 & 2.57 & 2.57 & 2.57 & 2.57 & 3.86 & 2.14 & 1.61 & 1.29 & 1.71 & 1.29 \\
\hline \multirow{2}{*}{ Class } & Combi & \multicolumn{6}{|c|}{ DWT-SO } & \multicolumn{6}{|c|}{ DWT-SZ } \\
\hline & Methods & SVM & ANN1 & ANNN2 & ANN3 & ANNN4 & ANNS & SVM & ANN1 & ANN2 & ANN3 & ANN4 & ANNS \\
\hline \multirow{3}{*}{ MC } & & 0.75 & 0.82 & 0.73 & 0.82 & 0.75 & 0.73 & 0.75 & 0.75 & 0.7 & 0.7 & 0.7 & 0.7 \\
\hline & & $(9,3)$ & $(9,2)$ & $(8,3)$ & $(9,2)$ & $(9,3)$ & $(8,3)$ & $(6,2)$ & $(6,2)$ & $(7,3)$ & $(7,3)$ & $(7,3)$ & $(7,3)$ \\
\hline & & 2.33 & 3.5 & 2.07 & 3.5 & 2.33 & 2.08 & 2.33 & 2.33 & 1.82 & 1.81 & 1.82 & 1.82 \\
\hline \multirow{3}{*}{$\mathrm{CR}$} & & 1.0 & 1.0 & 0.8 & 1.0 & 1.0 & 0.8 & 0.63 & 0.63 & 0.67 & 0.67 & 0.67 & 0.67 \\
\hline & DS & $(0,4)$ & $(0,5)$ & $(1,4)$ & $(0,5)$ & $(0,4)$ & $(1,4)$ & $(3,5)$ & $(3,5)$ & $(2,4)$ & $(2,4)$ & $(2,4)$ & $(2,4)$ \\
\hline & & 1.0 & 1.0 & 5.14 & 1.0 & 10 & 5.14 & 2.14 & 2.14 & 2.57 & 2.57 & 2.57 & 2.57 \\
\hline
\end{tabular}

\begin{tabular}{|c|c|c|c|c|c|c|c|c|c|c|c|c|c|}
\hline \multicolumn{14}{|c|}{ Cross-validation test 5} \\
\hline \multirow{2}{*}{ Class } & Combining & \multicolumn{6}{|c|}{ FFT } & \multicolumn{6}{|c|}{ DWT-CP } \\
\hline & & $\mathrm{SVM}$ & ANN1 & ANN2 & ANN 3 & ANN4 & ANN5 & SVM & ANN1 & ANNN2 & ANN 3 & ANN4 & ANN5 \\
\hline \multirow{3}{*}{ MC } & $\mathrm{BC}$ & 0.83 & 0.9 & 0.82 & 0.91 & 0.75 & 0.82 & 0.82 & 0.78 & 0.78 & 0.71 & 0.82 & 0.75 \\
\hline & DS & $(10,2)$ & $(9,1)$ & $(9,2)$ & $(10,1)$ & $(9,3)$ & $(9,2)$ & $(9,2)$ & $(7,2)$ & $(7,2)$ & $(5,2)$ & $(9,2)$ & $(6,2)$ \\
\hline & 10 & & 4.5 & 2.25 & 5.0 & 1.5 & 2.25 & 2.25 & 1.75 & 1.75 & 1.25 & 2.25 & 1.5 \\
\hline \multirow{3}{*}{$\mathrm{CR}$} & & & 8 & 0.75 & 1.0 & 0.67 & 0.75 & 0.75 & 0.5 & 0.5 & 0.38 & 0.75 & 0.43 \\
\hline & & $(0,3)$ & $(1,4)$ & $(1,3)$ & $(0,4)$ & $(1,2)$ & $(1,3)$ & $(1,3)$ & $(3,3)$ & $(3,3)$ & $(5,3)$ & $(1,3)$ & $(4,3)$ \\
\hline & & 0 & 8.0 & 6.0 & 1.0 & 4.0 & 6.0 & 6.0 & 2.0 & 2.0 & 1.2 & 6.0 & 1.5 \\
\hline \multirow{2}{*}{ Class } & Comb & & \\
\hline & \multicolumn{7}{|c|}{ 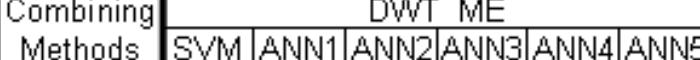 } & \multicolumn{6}{|c|}{$\begin{array}{l}\text { DWV-RA } \\
\text { ANN2 ANN3 ANN4 ANN5 }\end{array}$} \\
\hline \multirow{3}{*}{ MC } & & 0.83 & 1.0 & 0.9 & 0.9 & 0.83 & 0.82 & 0.7 & 0.63 & 0.67 & 0.6 & \begin{tabular}{|l|}
0.7 \\
\end{tabular} & 0.78 \\
\hline & & $(10,2)$ & $(10,0)$ & $(9,1)$ & $(9,1)$ & $(10,2)$ & $(9,2)$ & $(7,3)$ & $(5,3)$ & $(6,3)$ & $(3,2)$ & $(7,3)$ & $(7,2)$ \\
\hline & $\mathrm{L}$ & 2.5 & 0 & 4.5 & 4.5 & 2.5 & 2.25 & 1.17 & 0.83 & 1.0 & 0.75 & 1.17 & 1.75 \\
\hline & & & 1.0 & 0.8 & 0.8 & 1.0 & 0.75 & 0.4 & 0.29 & 0.33 & 0.3 & 0.4 & 0.5 \\
\hline & & 13) & $(0,5)$ & $(1,4)$ & $(1,4)$ & $(0,3)$ & $(1,3)$ & $(3,2)$ & $(5,2)$ & $(4,2)$ & $(7,3)$ & $(3,2)$ & $(3,3)$ \\
\hline & $\mathrm{LC}$ & 1.0 & 1.0 & 8.0 & 8.0 & 1.0 & 6.0 & 1.33 & 0.8 & 1.0 & 0.86 & 1.33 & 2.0 \\
\hline \multirow{2}{*}{ Class } & Combir & \multicolumn{6}{|c|}{ DWT-SO } & \multicolumn{6}{|c|}{ DWT-SZ } \\
\hline & & SVM & ANN1 & ANN2 & ANN 3 & ANN4 & ANN5 & $5 \mathrm{VM}$ & ANN1 & A.NN2 & ANNN3 & ANN4 & ANNS \\
\hline \multirow{3}{*}{ MC } & & 0.83 & 0.8 & 0.8 & 0.8 & 0.8 & 0.8 & 1.0 & 1.0 & \begin{tabular}{|l|}
0.9 \\
\end{tabular} & 0.9 & 0.83 & 0.82 \\
\hline & & $(10,2)$ & $(8,2)$ & $(8,2)$ & $(8,2)$ & $(8,2)$ & $(8,2)$ & $(10,0)$ & $(10,0)$ & $(9,1)$ & $(9,1)$ & $(10,2)$ & $(9,2)$ \\
\hline & & 2.5 & 2.0 & 2.0 & 2.0 & 2.0 & 2.0 & 0 & 0 & 4.5 & 4.5 & 2.5 & 2.25 \\
\hline \multirow{3}{*}{$\mathrm{CR}$} & $E$ & 1.0 & 0.6 & 0.6 & 0.6 & 0.6 & 0.6 & 1.0 & 1.0 & 0.8 & 0.8 & 1.0 & 0.75 \\
\hline & $\mathrm{DS}$ & $(0,3)$ & $(2,3)$ & $(2,3)$ & $(2,3)$ & $(2,3)$ & $(2,3)$ & $(0,5)$ & $(0,5)$ & $(1,4)$ & $(1,4)$ & $(0,3)$ & $(1,3)$ \\
\hline & & 1.0 & 3.0 & 3.0 & 3.0 & 3.0 & 3.0 & 1.0 & 1.0 & 8.0 & 8.0 & 1.0 & 6.0 \\
\hline
\end{tabular}




\section{Bibliography}

[1] K. M. Ali and M.J. Pazzani. Error reduction through Learning Multiple Descriptions. Machine Learning, 24(3):173-202, 1996.

[2] A. A. Anastassopoulos, V. N. Nikolaidis, and T. P. Philippidis. A Comparative Study of Pattern Recognition Algorithms for Classification of Ultrasonic Signals. Neural Computing \& Applications, 8:53-66, 1999.

[3] S. Bae, L. Udpa, and S. Udpa. Classification of Ultrasonic Weld Inspection Data Using Principal Component Analysis. Review of Progress in Quantitative Nondestructive Evaluation, 16:741-748, 1997.

[4] D. Bahler and L. Navarro. Methods for combining heterogeneous sets of classifiers. In Proceedings of the 17th Natl. Conf. on Artificial Intelligence (AAAI), Workshop on New Research Problems for Machine Learning, 2000.

[5] D. Berry, L. Udpa, and S. S. Udpa. Classification of Ultrasonic Signals via Neural Networks. Review of Progress in Quantitative Nondestructive Evaluation, 10A:659666, 1991.

[6] W. E. Bond, D. C. St. Clair, M. M. Amirfathi, C. J. Merz, and S. Aylward. Neural Network Analysis of Nondestructive Evaluation Patterns. In Proceedings of the 1992 ACM/SIGAPP Symposium on Applied Computing, volume 2, pages 643-650. ACM Press, 1992.

[7] B. Boser, I. Guyon, and V. Vapnik. A training algorithm for optimal margin classifiers. In Proceedings of the 5th Annual ACM Workshop on Computational Learning Theory, pages 144-152. ACM Press, 1992. 
[8] P. Brazdil, J. Gama, and R. Henery. Characterizing the Applicability of Classification Algorithms using Meta Level Learning. In Proceedings of the European Conference on Machine Learning, ECML-94, pages 83-102, 1994.

[9] L. Breiman. Bagging Predictors. Machine Learning, 24(2):123-140, 1996.

[10] G.J. Briem, J.A. Benediktsson, and J.R. Sveinsson. Boosting, Bagging and Consensus Based Classification of Multisource Remote Sensing Data. In J. Kitter and F. Roli, editors, Multiple Classifier Systems. Second International Workshop, MCS 2001, Lecture Notes in Computer Science 2096, pages 279-288. Springer-Verlag, 2001.

[11] E. O. Brigham. The Fast Fourier Transform. Prentice Hall, 1997.

[12] L. M. Brown and R. DeNale. Classification of Ultrasonic Defect Signatures Using An Artificial Neural Network. Review of Progress in Quantitative Nondestructive Evaluation, 10A:705-712, 1991.

[13] Christopher J.C. Burges. A tutorial on Support Vector Machines for Pattern Recognition. Data Mining and Knowledge Discovery, 2:121-167, 1998.

[14] T. J. Case and R. C. Waag. Flaw Identification from Time and Frequency Features of Ultrasonic Waveforms. IEEE Transactions on Ultrasonics, Ferroelectrics, and Frequency Control, 43(4):592-600, 1996.

[15] Chih-Chung Chang and Chih-Jen Lin. LIBSVM: a library for support vector machines, 2001. Software available at http://www.csie.ntu.edu.tw/ cjlin/libsvm.

[16] R. Ciocan, D. Ciobanu, and W. R. Mayo. Neural Network Classification of Flaws Detected During Ultrasonic Inspection of Candu Pressure Tubes and Brazed Joints in Candu Fuel. In Proceedings of ECNDT '98, [Online], 1998.

[17] R. Ciocan, P. Petulescu, D. Ciobanu, and D. J. Roth. The use of the neural networks in the recognition of the austentic steel types. NDT $\&$ E International, 33:85-89, 2000 .

[18] P. Clark and R. Boswell. Rule Induction with CN2: Some Recent Improvements. In Proceedings of the European Working Session on Learning, pages 151-163. Pitman, 1991. 
[19] G.M. Clarke and D. Cooke. A Basic Course in Statistics. Arnold, 1998.

[20] P.R. Cohen. Empirical Methods for Artificial Intelligence. MIT Press, 1995.

[21] J. M. et.al. Combes. Wavelets, Time-Frequency Methods and Phase Space. SpringerVerlag, 1989.

[22] C. Cortes and V. Vapnik. Support Vector Networks. Machine Learning, 20:273-297, 1995.

[23] G. Cotterill and J. Perceval. A New Approach to Ultrasonic Testing of Shafts. In Proceedings of the 10th Asia-Pacific Conference on Non-Destructive Testing (APCNDT), [Online], 2001.

[24] N. Cristianini and J. Shawe-Taylor. An Introduction to Support Vector Machines and other kernel-based learning methods. Cambridge University Press, 2000.

[25] B. V. Dasarathy and B. V. Sheela. A composite classifier system design: concepts and methodology. In Proceedings of IEEE, volume 67, pages 708-713. IEEE Press, 1978.

[26] I. Daubechies. Orthogonal bases of compactly supported wavelets. Communications on Pure and Applied Mathematics, 41:909-996, 1988.

[27] I. Daubechies. IEEE Trans. Inform. Theory. The Wavelet Transform, Time-Frequency Localization and Signal Analysis, 36:961-1005, September 1990.

[28] D.J. DeFatta, J.G. LUcas, and W.S. Hodgkiss. Digital Signal Processing. Wiley, 1988.

[29] T. G. Dietterich. Machine-learning research: Four current directions. AI Magazine, 18(4):97-136, 1997.

[30] R. Duda, J. Gasching, and P. Hart. Model Design in the Prospector Consultant System for Mineral Exploration. In D. Michie, editor, Expert Systems in the MicroElectronic Age, pages 153-167. Edinburgh University Press, Edinburgh, 1979.

[31] R.P.W. Duin. The combining classifier: to train or not to train? In Proceedings of the 16th International Conference on Pattern Recognition, ICPR'02, pages 765-770, 2002. 
[32] B. Efron and R. Tibshirani. An introduction to the Bootstrap. Chapman and Hall, New York, 1993.

[33] Aida A. Ferreira, Teresa B. Ludermir, and Ronaldo R.B. de Aquino. Comparing Neural Network Architecture for Pattern Recognize System on Artifical Noses. Lecture Notes in Computer Science, 3696/2005(7):635-640, 2005.

[34] C. Gold and P. Sollich. Model selection for support vector machine classification. Neurocomputing, 55:221-249, 2003.

[35] C. E. Heil and D. F. Walnut. SIAM Review. Continuous and Discrete Wavelet Transforms, 31:628-666, December 1989.

[36] T. K. Ho. Multiple classifier combination: Lessons and the next steps, pages 171-198. World Scientific Publishing, 2002.

[37] C.-W. Hsu, C.-C. Chang, and C.-J. Lin. A practical guide to support vector classification. Available at http://www.csie.ntu.edu.tw/ cjlin/papers/guide/guide.pdf.

[38] D. K. Iakovidis, D. E. Maroulis, S. A. Karkanis, and I. N. Flaounas. Color Texture Recognition in Video Sequences using Wavelet Covariance Features and Support Vector Machines. In Proceedings of the 29th Euromicro Conference, pages 199-205, 2003.

[39] A. Kalousis and M. Hilario. Model Selection via Meta-learning: a Comparative Study. In Proceedings of the 12th International IEEE Conference on Tools with AI. IEEE press, 2000.

[40] S. V. Kamarthi and S. Pittner. Fourier and Wavelet Transform for Flank Wear Estimation - A Comparison. Mechanical Systems and Signal Processing, 11(6):791809, 1997.

[41] M. S. Kamel and N. M. Wanas. Data dependence in combining classifiers. In T. Windeatt and PUBLISHER $=$ Springer-Verlag Roli, F.) YEAR $=2003$, editors, Proceedings of the 4th International Workshop on Multiple Classifier Systems(MCS2003), volume 2709, pages 1-14. 
[42] D. A. Karras, S. A. Karkanis, D. Iakovidis, D.E. Maroulis, and B.G. Mertzios. Support Vector Machines for Improved Defect Detection in Manufacturing Using Novel Multidimensional Wavelet Feature Extraction Involving Vector Quantization and PCA Techniques. In Proceedings of NIMIA Advanced Study Institute on Neural Networks for Instrumentation, Measurement and Related Industrial Applications, pages 139$144,2001$.

[43] G. Katragadda, S. Nair, and G. P. Singh. Neuro-Fuzzy Systems in Ultrasonic Weld Evaluation. Review of Progress in Quantitative Nondestructive Evaluation, 16:765$772,1997$.

[44] S. M. Kay. Modern Spectral Estimation. Prentice-Hall, 1988.

[45] S. S. Keerthi and C. J. Lin. Asymptotic behaviors of support vector machines with Gaussian kernel. Neural Computation, 15(7):1667-1689, 2003.

[46] S. Sathiya Keerthi. Efficient Tuning of SVM Hyperparameters Using RadiusMargin Bound and Iterative Algorithms. IEEE Transactions on Neural Networks, 13(5):12251229, September 2002.

[47] V. Khandetsky and I. Antonyuk. Signal Processing in defect detection using backpropagation neural networks. NDT \& E International, 35:483-488, 2002.

[48] B. Ko and H. Byun. Probalilistic Neural Networks Supporting Multi-Class Relevance Feedback in Region-Based Image Retrieval. In Proceedings of the 16th International Conference on Pattern Recognition (ICPR-02), volume 4, pages 4013-4018. IEEE Computer Society, 2002.

[49] J. Kräutkramer. Ultrasonic Testing of Materials. Springer-Verlag, Germany, 1969.

[50] R. Kronland-Martinet, J. Morlet, and A. Grossmann. Analysis of Sound Patterns Through Wavelet Transforms. International Journal of Pattern Recognition and Artificial Intelligence, 1:273-301, 1987.

[51] W. H. Kruskal and J. M. Tanur. International encyclopedia of statistics. NY:Free Press, 1978.

[52] R. Kuc. Introduction to Digital Signal Processing. McGraw-Hill, 1988. 
[53] Ludmila I. Kuncheva. Combining Pattern Classifiers: Methods and Algorithms. John Wiley and Sons, Inc., 2004.

[54] L. Lam. Classifier combinations: Implementations and theoretical issues. In J. Kittler and F. Roli, editors, Multiple Classifier Systems, pages 78-86. Springer-Verlag, 2000.

[55] S. Legendre, D. Massicotte, J. Goyette, and T. K. Bose. Wavelet-Transform-Based Method of Analysis for Lamb-Wave Ultrasonic NDE Signals. In Proceedings of the IEEE Instrumentation \& Measurement Technology, pages 860-865, 1999.

[56] H. T. Lin and C. J. Lin. A study on sigmoid kernels for svm and the training of non-psd kernels by smo-type methods. Available at http://www.csie.ntu.edu.tw/ cjlin/papers/tanh.pdf.

[57] F. Lingvall and T. Stepinski. Automatic detecting and classifying defects during eddy current inspection of riveted lap-joints. NDT \& E International, 33:47-55, 2000.

[58] P.A. Lynn and W. Fuerst. Digital Signal Processing with computer applications. John Wiley, New York, 1994.

[59] J. Ma and S. Ahalt. OSU-SVM: SVM Classifier Matlab Toolbox (ver 2.0), 2001. Software available at http://eewww.eng.ohio-state.edu/maj/osu_svm/.

[60] S. Mallat. A theory for multiresolution signal decomposition: the wavelet representation. IEEE Transactions on Pattern Analysis and Machine Intelligence, 11(7):674$693,1989$.

[61] Y. Mallet, D. Coomans, J. Kautsky, and O. D. Vel. Classification Using Adaptive Wavelets for Feature Extraction. IEEE Transactions of Pattern Analysis and Machine Intelligence, 19(10):1058-1066, October 1997.

[62] F. W. Margrave, K. Rigas, D. A. Bradley, and P. Barrocliffe. The use of neural networks in ultrasonic flaw detection. Measurement, 25:143-154, 1999.

[63] S. L. Marple. Digital Spectral Analysis. Prentice-Hall, 1987.

[64] A. Masnata and M. Sunseri. Neurual network classification of flaws detected by ultrasonic means. NDT \& E International, 29(2):87-93, 1996. 
[65] J. H. McClellan, R. W. Schafer, and M. A. Yoder. DSP First A multimedia approach. Prentice Hall, 1998.

[66] T. Mitchell. Machine Learning. McGraw Hill, 1997.

[67] S. H. Nawab and T. F. Quatieri. Short-Time Fourier Transform. 1988.

[68] H. J. Nussbaumer. Fast Fourier Transform and Convolution Algorithms. SpringerVerlag, 1981.

[69] M. S. Obaidat, M. A. Suhail, and B. Sadoun. An intelligent simulation methdology to characterize defects in materials. Information Sciences, 137:33-41, 2001.

[70] S. Ogaji and R. Singh. Advanced engine diagnostics using artificial neural networks. Applied Soft Computing, 3(3):259-271, November 2003.

[71] D. Opitz. Feature selection for ensembles. In Proceedings of the 16th National Conference on Artificial Intelligence, pages 379-384. AAAI Press, 1999.

[72] E. Osuna, R. Freund, and F. Girosi. Training support vector machines:an application to face detection. In Proceedings of Conference on Computer Vision and Pattern Recognition (CVPR 97), pages 130-136, 1997.

[73] S. Pittner and S. V. Kamarthi. Feature Extraction From Wavelet Coefficients for Pattern Recognition Tasks. IEEE Transactions on Pattern Analysis and Machine Intelligence, 21(1):83-88, January 1999.

[74] R. Polikar, L. Udpa, S. S. Udpa, and T. Taylor. Frequency Invariant Classification of Ultrasonic Weld Inspection Signals. IEEE Transactions on Ultrasonics, Ferroelectrics, and Frequency Control, 45(3):614-625, May 1998.

[75] M. R. Portnoff. IEEE Trans. on ASSP. Time-Frequency Representaiton of Digital Signals and Systems Based on the Short-Time Fourier Analysis, 28:55-69, February 1980.

[76] B. P. C. Rao, B. Raj, T. Jayakumar, and P. Kalyanasundaram. An artificial neural network for eddy current testing of autenitic stainless steel welds. NDT $\mathscr{E} E$ International, 35:393-398, 2002. 
[77] K. R. Rao. Discrete Transforms and their Applications. Van Nostrand Reinhold, New York, 1985.

[78] D. Redouane, K. Mohamed, and B. Amar. The Investigation of Artificial Neural Network Pattern Recognition of Acoustic Emission Signals for Pressure Vessel. In Proceedings of the 15th World Conference on Non-Destructive Testing, [Online], 2000.

[79] O. Rioul and M. Vetterli. IEEE Signal Processing Magazine. Wavelets and Signal Processing, 8:14-38, October 1991.

[80] B. Sadoun. An efficient simulation scheme for testing materials in a nondestructive manner. Information Sciences, 137:43-51, 2001.

[81] J. B. Santos and F. Perdigão. Automatic defects classification - a contribution. NDT E E International, 34:313-318, 2001.

[82] R. J. Schalkoff. Pattern Recognition: Statistical, Structural and Neural Approaches. New York: John Wiley and Sons, 1992.

[83] L. W. Schemerr Jr and S. J. Song. The Use of Probabilistic Neural Networks for Discontinuity Classification Problems. Materials Evaluation, pages 80-84, January 2000.

[84] M. Seeger. Bayesian model selection for Support Vector machines, Gaussian processes and other kernel classifiers. In Proceedings of the Neural Information Processing Systems, volume 12, pages 603-609. MIT Press, 2000.

[85] G. Shen, Q. Duan, B. Li, and Q. Liu. Flaw Detection in Ultrasonics Using Wavelets Transform and Split Spectrum. In Proceedings of the 15th World Conference on NonDestructive Testing, [Online], 2000.

[86] G. Simone, F. C. Morabito, R. Polikar, P. Ramuhalli, L. Udpa, and S. Udpa. Feature extraction techniques for ultrasonic signal classification. In Proceedings of the 10th Int. Symposium on Applied Electromagnetics and Mechanics (ISEM 2001), 2001.

[87] S. Song and Y. K Shin. Eddy current flaw characterization in tubes by neural networks and finite element modeling. NDT \& E International, 33:233-243, 2000. 
[88] S. J. Song, H. J. Kim, and H. Cho. Development of an intelligent system for ultrasonic flaw classification in weldments. Neuclear Engineering and Design, 212:307-320, 2002.

[89] S. J. Song, H. J. Kim, and H. Lee. A systematic approach to ultrasonic pattern recognition for real-time intelligent flaw classification in weldments. Review of Progress in Quantitative Nondestructive Evaluation, 18:865-872, 1999.

[90] J. Spanner, L. Udpa, R. Polikar, and P. Ramuhalli. Neural networks for ultrasonic detection of intergranular stress corrosion cracking. The e-Journal of Nondestructive Testing And Ultrasonics, 5(7), [Online], July 2000.

[91] D. F. Specht. Probabilistic Neural Networks For Classification, Mapping, or Associative Memory. In Proceedings of the IEEE International Conference on Neural Networks, pages 525-532, 1988.

[92] C. Suen and L. Lam. Multiple classifier combination methodologies for different output levels. In Multiple Classifier Systems. First International Workshop, MCS 2000, Lecture Notes in Computer Science 1857, pages 52-66. Springer-Verlag, 2000.

[93] H. C. Sun and J. Saniie. Orthogonal signal decomposition coupled with Bayes and Fuzzy discriminants classifiers for ultrasonic flaw detection. In Proceedings of the 1997 IEEE Ultrasonic Symposium, pages 639-642. IEEE Computer Society, 1997.

[94] H. Takada, A. Torao, M. Kitahama, T. Morii, R. Sugimoto, and A. Sato. Development of Roll Surface Testing Technique by use of Broad Bandwidth Surface Waves. In Proceedings of the 15th World Conference on Non-Destructive Testing, pages 291294, [Online], 2000.

[95] C. Mekdeci Tansel, I. N. and C. McLaughlin. Detection of Tool Failure in End Milling with Wavelet Transformations and Neural Networks (WT-NN). Machine Tools and Manufacture, 35(1):137-147, Aug. 1995.

[96] M. Thavasimuthu, C. Rajagopalan, P. Kalyanasundaram, and B. Raj. Improving the evaluation sensitivity of an ultrasonic pulse echo technique using a neural network classifier. NDT \& E International, 29(3):175-179, 1996.

[97] R. Thompson and D. Thompson. Ultrasonics in nondestructive evaluation. In Proceedings of IEEE, volume 73, pages 1716-1755, 1995. 
[98] G. Valentini and F. Masulli. Ensembles of Learning Machines. In R. Tagliaferri and M. Marinaro, editors, Neural Nets WIRN vietri-2002, Lecture Notes in Computer Science 2486, pages 3-19. Springer-Verlag, 2002.

[99] V. N. Vapnik. Estimation of Dependencies Based on Empirical Data. Springer-Verlag, 1982.

[100] V. N. Vapnik. The Nature of Statistical Learning Theory. New York: Springer, 1995.

[101] D. H. Wolpert. Stacked Generalization. Neural Network, 5:241-259, 1992.

[102] K. Woods, W. P. Kegelmeyer, and K. Bowyer. Combination of multiple classifiers using local accuracy estimates. IEEE Transactions on Pattern Analysis and Machine Intelligence, 19:405-410, 1997.

[103] Y. Xiang and S. K. Tso. Detection and classification of flaws in concrete structure using bispectra and neural networks. NDT \& E International, 35:19-27, 2002.

[104] L. Xu, C. Krzyzak, and C. Suen. Methods of combining multiple classifiers and their applications to handwriting recognition. IEEE Transactions on Systems, Man and Cybernetics, 22(3):418-435, 1992.

[105] A. Zaknich. Introduction to the modified probabilistic neural network for general signal processing applications. IEEE Transactions on Signal Processing, 46(7):19801990, July 1998.

[106] A. Zell. SNNS: Stuttgart Neural Network Simulator. University of Stuttgart, Germany, 1991. Software available at http://www_ra.informatik.uni_tuebingen.de/SNNS/.

[107] F. Zhu, S. U. Guan, and P. Li. Feature Selection for Modular GA-based Classification. Applied Soft Computing, 4(4), September 2004. 